\title{
MATHEMATICAL MODELS OF INFLUENZA A VIRUS INFECTIONS IN VITRO: INVESTIGATING DEFECTIVE INTERFERING PARTICLES AND VIRUS RELEASE
}

\author{
by \\ Laura Liao \\ M.Sc. University of British Columbia, Vancouver (BC), Canada, 2013 \\ B.Sc. Ryerson University, Toronto (ON), Canada, 2011 \\ A dissertation \\ presented to Ryerson University \\ in partial fulfillment of the \\ requirements for the degree of \\ Doctor of Philosophy \\ in the program of \\ Biomedical Physics
}

Toronto, Ontario, Canada, 2017

(c) Laura Liao 2017 


\section{Author's Declaration}

I hereby declare that I am the sole author of this dissertation. This is a true copy of the dissertation, including any required final revisions, as accepted by my examiners.

I authorize Ryerson University to lend this dissertation to other institutions or individuals for the purpose of scholarly research.

I further authorize Ryerson University to reproduce this dissertation by photocopying or by other means, in total or in part, at the request of other institutions or individuals for the purpose of scholarly research.

I understand that my dissertation may be made electronically available to the public. 


\begin{abstract}
Mathematical Models of Influenza A Virus Infections in Vitro: Investigating Defective Interfering Particles and Virus Release Laura Liao

Doctor of Philosophy, Biomedical Physics

Ryerson University, 2017
\end{abstract}

In this work, two studies were performed where mathematical models (MM) were used to re-examine and refine quantitative methods based on in vitro assays of influenza A virus infections.

In the first study, we investigated the standard experimental method for counting defective interfering particles (DIPs) based on the reduction in standard virus (STV) yield (Bellett \& Cooper, 1959). We found the method is valid for counting DIPs provided that: (1) a STV-infected cell's co-infection window is approximately half its eclipse phase (it blocks infection by other virions before it begins producing progeny virions); (2) a cell co-infected by STV and DIP produces less than 1 STV per 1,000 DIPs; and (3) a high MOI of STV stock (>4 plaque-forming units/cell) is added to perform the assay. Prior work makes no mention of these criteria such that the counting method has been applied incorrectly in several publications discussed herein. We determined influenza A virus meets these criteria, making the method suitable for counting influenza A DIPs.

In the second study, we compared a MM with an explicit representation of viral release to a simple MM without explicit release, and investigated whether parameter estimation and the estimation of neuraminidase inhibitor (NAI) efficacy were affected by the use of a simple MM. Since the release rate of influenza A virus is not well-known, a broad range of release rates were considered. If the virus release rate is greater than $\sim 0.1 \mathrm{~h}^{-1}$, the simple MM provides accurate estimates of infection parameters, but underestimates NAI efficacy, which could lead to underdosing and the emergence of NAI resistance. In 
contrast, when release is slower than $\sim 0.1 \mathrm{~h}^{-1}$, the simple MM accurately estimates NAI efficacy, but it can significantly overestimate the infectious lifespan (i.e., the time a cell remains infectious and producing free virus), and it will significantly underestimate the total virus yield and thus the likelihood of resistance emergence. We discuss the properties of, and a possible lower bound for, the influenza A virus release rate.

Overall, MMs are a valuable tool in the exploration of the known unknowns (i.e., DIPs, virus release) of influenza A virus infection. 


\section{Acknowledgements}

First, I thank my supervisor Dr. Catherine Beauchemin for guiding my research, as well as providing encouragement, and constantly stoking my enthusiasm for the influenza A virus throughout my time here. I would like to acknowledge the Natural Sciences and Engineering Research Council of Canada for providing funding for this work in the form of a Doctoral Canadian Graduate Scholarship and Michael Smith Foreign Study Supplement. Additionally, I would like to acknowledge funding support in the form of an Ontario Graduate Scholarship from the Government of Ontario, as well as support in the form of the Doctoral Completion award from Ryerson University. 


\title{
Table of Contents
}

\author{
Author's Declaration
}

Abstract

Acknowledgements $\quad$ v

List of Tables $\quad$ ix

List of Figures $\quad x$

1 Introduction 1

2 Background 4

2.1 Influenza A virus infection . . . . . . . . . . . . . . . . . . 4

2.1 .1 Virus structure . . . . . . . . . . . . . 4

2.1.2 Virus replication cycle . . . . . . . . . . . . . 6

2.1.3 Antiviral therapies . . . . . . . . . . . . . . . 10

2.2 Previous mathematical models _. . . . . . . . . . . . . . . 12

3 (In)validating experimentally-derived knowledge about influenza A $\begin{array}{ll}\text { defective interfering particles } & 15\end{array}$

3.1 Introduction . . . . . . . . . . . . . . . . . 16

3.2 Results. . . . . . . . . . . . . . . . . . . . 21 
3.2.1 Theoretical basis and failure of the Bellett \& Cooper assay . . . . 21

3.2.2 A mathematical model of influenza A STV and DIP infection kinetics 23

3.2 .3 Effect of the co-infection window . . . . . . . . . . 27

3.2.4 Length of an influenza A virus co-infection window . . . . . . . . . 29

3.2.5 Effect of allowing co-infected cells to produce progeny STV . . . . 29

3.2.6 STV production by cells co-infected by influenza A STV and DIP 32

3.2.7 Effect of using STV stock at low MOI to perform the B\&C assay . 33

3.3 Discussion . . . . . . . . . . . . . . . . . 36

\section{Exploring virus release as a bottleneck for the spread of influenza A} $\begin{array}{ll}\text { virus infection in vitro } & 41\end{array}$

4.1 Introduction . . . . . . . . . . . . . . . . . . . 42

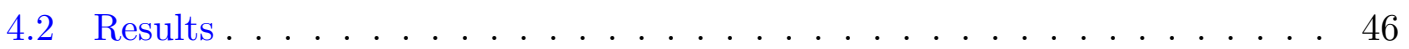

4.2.1 Considering an explicit release rate and the impact on viral kinetics 46

4.2.2 The effect of explicit virus release on parameter estimates . . . . . 48

4.2.3 Characterizing NAI efficacy in the context of explicit vs implicit release ........................ 52

4.2.4 Exploring the difference between NAIs and polymerase inhibitors in the context of the release MM . . . . . . . . . . . 53

4.3 Discussion . . . . . . . . . . . . . . . . . . . . 55

4.4 Methods . . . . . . . . . . . . . . . . . . . . . . 59

4.4.1 Mathematical models of influenza A viral infection kinetics . . . . 59

4.4.2 The effective production rate in the release MM . . . . . . . . 61

4.4 .3 Modelling antiviral effect . . . . . . . . . . . . . . 62

4.4.4 Numerically solving transformed equations . . . . . . . . . . . 63

4.4.5 Simulation of infections . . . . . . . . . . . . . . . 64

4.4 .6 Treatment with antivirals . . . . . . . . . . . . 66 
$\begin{array}{lll}\text { Appendix A } & 71\end{array}$

A.1 Biological basis of key co-infection parameters of influenza A virus . . . . 71

A.2 Alternative explanation for the reversal of DIP-mediated interference . . . 72

A.3 Sensitivity of the $\mathrm{B} \& \mathrm{C}$ assay to samples containing very high or very low

DIP MOIs . . . . . . . . . . . . . . . . . . 75

A.4 Classification of types and causes of curvature in the B\&C assay . . . . . 76

A.5 Methods . . . . . . . . . . . . . . . . 78

A.5.1 The multiplicity of infection (MOI) determines the fraction of infected cells ....................... 78

A.5.2 Mathematical model . . . . . . . . . . . . . . . . 78

A.5.3 Simulating infections . . . . . . . . . . . . . . . 80

$\begin{array}{lll}\text { Appendix B The release MM } & 85\end{array}$

B.1 Determination of critical release rates . . . . . . . . . . . . 85

B.2 Infection parameters predicted by the release MM $\ldots \ldots \ldots$. . . . 88

$\begin{array}{ll}\text { Bibliography } & 90\end{array}$

$\begin{array}{ll}\text { Glossary } & 101\end{array}$ 


\section{List of Tables}

4.1 Base parameter values for infection with an influenza A (H1N1) pandemic strain $[85] \ldots \ldots \ldots \ldots \ldots \ldots$

A.1 Base parameter values for infection with an influenza A (H1N1) pandemic strain $[81] \ldots \ldots \ldots \ldots$. . . . . . . . . . . . . . . . . . . 80

A.2 Best fit parameters to Nayak's time of DIP addition data . . . . . . . . 82

A.3 Best fit parameters to Akkina's reversal of DIP-mediated interference data 84 


\section{List of Figures}

2.1 Structure of influenza A virion . . . . . . . . . . . . . . . 5

2.2 Influenza A virus replication cycle . . . . . . . . . . . . 8

2.3 Viral polymerase acts as both transcriptase and replicase . . . . . . . . 9

2.4 Modes of action of antivirals . . . . . . . . . . . . . . . 11

3.1 Process of standard virus yield suppression by DIPs . . . . . . . . . . 17

3.2 The presence of DIPs is only evident at high STV MOI . . . . . . . . . 19

3.3 Counting of DIPs in the Bellett \& Cooper 1959 assay . . . . . . . . . . . 22

3.4 Experimental deviation from the theoretical predictions of Bellett \& Cooper 24

3.5 Mathematical model for the kinetics of influenza A virus infection in the presence of DIPs . . . . . . . . . . . . . . . 25

3.6 Impact of the co-infection window in the B\&C assay . . . . . . . . . . . 28

3.7 The length of the co-infection window for influenza A virus . . . . . . . 30

3.8 Impact of non-negligible STV yield by co-infected cells in the B\&C assay 31

3.9 STV produced by cells co-infected with influenza A STV and DIPs . . . . 32

3.10 Impact of the STV MOI of the DIP-free STV stock . . . . . . . . . . . . 35

4.1 Modelling influenza A virus infection with and without explicit release . . 44

4.2 The role of explicit viral release on free and bound virus kinetics . . . . . 47

4.3 Simultaneous fits of the release MM to the simulated free virus titer of the SC, MC, MY assays . . . . . . . . . . . . . . . . 49

4.4 Comparing parameter estimates between the simple MM and release MM 50

4.5 Simultaneous fits of the release MM to the simulated SC, MC, MY titers with a constraint on virus production . . . . . . . . . . . 52

4.6 Comparing the effect of NAIs in the simple and release MMs . . . . . . . 54

4.7 Comparing antivirals that inhibit release to antivirals that inhibit production in the release MM . . . . . . . . . . . . . . 55

4.8 Instabilities in numerically solving model ordinary differential equations

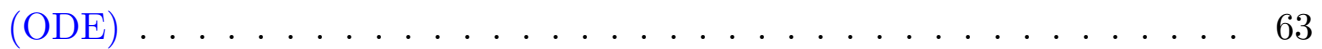

4.9 Comparing the $\mathrm{AIC}_{\mathrm{C}}$ of release $\mathrm{MM}$ fits $\ldots \ldots \ldots 6$

A.1 Evidence by Akkina et al. for a reversal of DIPs' interfering, suppressive effect . . . . . . . . . . . . . . . . . 73

A.2 Impact of the DIP MOI of the sample on the B\&C assay . . . . . . . . 76

A.3 Classifying types and causes of non-exponential curvature in the $\mathrm{B} \& \mathrm{C}$ assay 77 
A.4 Evaluating the reliability of the co-infection window estimated from the Nayak et al. assay . . . . . . . . . . . . . . . . . . 83

B.1 Determination of critical free and bound virus release rates, $r_{f}$ and $r_{b} \quad \ldots \quad 86$

B.2 The critical free virus release rate, $r_{f}$, weakly depends on infection parameters . . . . . . . . . . . . . . . . . 87

B.3 The critical bound virus release rate, $r_{b}$, depends on infection parameters 88

B.4 The simple MM does not significantly misestimate the infection rate, rate of loss of virion infectivity, or the basic reproductive number . . . . . . . 89 


\section{Chapter 1}

\section{Introduction}

Influenza is an acute respiratory infection that has no cure, recurring every year with significant impact on human health around the world. Influenza is estimated to cause 3-5 million cases of severe illness, and 250,000-500,000 deaths during annual epidemics globally [111]. In Canada alone, there were over 38,000 reported cases of influenza in the 2016-2017 flu season [88]. Of these cases, 90\% were cases of influenza A virus infections.

Influenza is caused by 3 types of influenza virus (A, B, and $\mathrm{C}$ ). Of least significant risk to public health are influenza $\mathrm{C}$ viruses, which cause mild illness, and therefore receives the least attention. Compared to influenza B viruses, influenza A viruses cause epidemics more frequently (every year vs. 2-4 years). As well, influenza A virus remains the only type with pandemic potential [72]. In this thesis, influenza A virus infections are studied.

Influenza A viruses can infect a range of hosts including humans, birds, swine, horses, and dogs [112]. In humans, the influenza A virus infects epithelial cells that line the upper respiratory tract. An uncomplicated influenza A viral infection is characterized by symptoms such as headache, chills, dry cough, fever and muscle pain. Symptoms tend to resolve within 3-7 days, but a healthy adult can infect others one day before the onset of symptoms and up to 5-7 days after resolution [20]. Transmission of the virus occurs from person to person through large particle droplets that are emitted from coughing and sneezing. Transmission can also occur through contact with contaminated surfaces. The influenza A virus is highly contagious and spreads rapidly throughout the population, and around the world [5, 11, 94].

Efforts to prevent and treat influenza A virus infection are constantly thwarted by the rapid evolution of influenza A virus strains [45]. In order to prevent illness, there is a race to formulate vaccines that correctly match the ever-changing antigens of circulating 
seasonal strains. Once infected, treatment with antivirals can be administered to lessen the severity of the infection. However, influenza A virus strains often acquire mutations that confer resistance to such antivirals. To make matters worse, there is the looming threat that every 10 to 40 years, a strain might emerge to which humans have no preexisting immunity to, causing a pandemic event that could attack $20 \%$ to $40 \%$ of the world population [112]. In 1918, Spanish influenza was the most devastating pandemic recorded, killing up to 25 million people in 25 weeks. Three major pandemics have been recorded since then, for example, Asian influenza in 1958, Hong Kong influenza in 1968, and pandemic H1N1 in 2009. For these reasons, the influenza A virus has been studied to not only gain a better understanding of its life cycle, but to produce a variety of interventions to reduce its spread and burden.

It is in this context that mathematical models (MM) have long been used to study the dynamics of influenza A virus at the population level, focussing on transmission between individuals. In particular, MMs have been used to inform public health decisions in pandemic planning [28]. For example, MMs have been used to explore social distancing, the use of antivirals, and immunization as strategies to mitigate transmission [70]; as well as to guide data collection and to prioritize certain demographics for vaccination [61]. Complementing this view of infection between individuals, are studies of influenza A virus infection within an individual, which MMs have only just begun to be used within the past decade.

As surveyed by Beauchemin et al. (and reviewed in [8, 99]), within-host MMs of influenza infection have been used to gain conceptual insights to understand the system, test specific mechanistic hypotheses, and quantify kinetic parameters of the system to make quantitative comparisons (e.g., between virus strains) [3]. Beauchemin et al. further suggest that such studies will play an important role in the creation of the ultimate predictive tool: a multiscale MM that can predict complex phenotypes such as virulence and transmissibility, given the genotype sequence of a virus strain. This illustrates the study of infection kinetics at various spatial scales, e.g., in a single cell (intracellularly), within a cell culture (in vitro), within the respiratory tract of individuals such as humans or animal models (in vivo), and between individuals. While MMs of intracellular systems must consider many individual components such as viral proteins, viral gene segments, numerous steps in viral replication [22, 34, 60], and MMs of in vivo infections must consider cell tropism [16] and host immune responses [18, 33], in comparison, MMs of in vitro systems are simpler and free from such complexities. In and of itself, in vitro systems present many additional advantages, i.e., they can be used to study highly virulent strains like avian influenza virus which cannot be studied in humans, they are 
low-cost, reproducible, and do not suffer from low statistical confidence compared to animal models. Hence, we focus on the analysis of influenza A virus infections in vitro using MMs.

Key quantitative insights and predictions have been obtained from mathematical modelling of in vitro systems. For example, MMs have been used to explain the differences between the replicative capacities of an oseltamivir-resistant seasonal A/H1N1 strain and a previous seasonal strain in vitro [39], to translate the effects of specific mutations in pandemic A/H1N1 into its replicative capacity in vitro [81, 85], to compare replication properties of avian viruses to human viruses [98], and to quantitatively characterize antiviral efficacy [4]. Our work is situated in this landscape, where with the help of MMs, we aim to re-examine and refine quantitative methods that are based on such in vitro assays of influenza A virus infection.

In the first half of this work, I investigate an in vitro assay used to count defective interfering particles (DIP) of influenza A virus. The DIP counting assay was developed in 1959, and is still used to this day. With the MM, we establish a set of criteria that has never before been described for this assay, yet must be followed in order to produce valid results. In the second half of this work, I investigate influenza A virus release and representations of it in a MM (i.e., explicitly or implicitly). Current MMs with an implicit representation of virus release have been used to quantify influenza A virus infection kinetics parameters and estimate the antiviral efficacy of neuraminidase inhibitors. We show that the interpretation of the results obtained from such a MM depends on the magnitude of the influenza A virus release rate. We highlight the importance of quantifying the influenza $A$ virus release rate and the implications of an implicit representation of virus release in a MM.

In Chapter 2, the biology of the influenza A virus is discussed, including a detailed explanation of the virus replication cycle and how antivirals disrupt stages of the replication cycle. A brief summary of MMs previously used to study DIPs or virus release is also given. In Chapter 3 and Chapter 4, we present our main findings and discuss the implications. 


\section{Chapter 2}

\section{Background}

In this chapter, we review the basic biology of influenza A virus including its structure, the virus replication cycle, and the modes of action of antivirals used in the treatment of influenza A virus infection. This will provide sufficient information to understand the influenza A virus-cell interactions that are represented in our MMs. Lastly, we summarize previous MMs that have been used to investigate defective interfering particles in general, and influenza A virus release specifically.

\subsection{Influenza A virus infection}

\subsubsection{Virus structure}

An influenza A virus particle is spherical, approximately $80 \mathrm{~nm}-120 \mathrm{~nm}$ in diameter [93], but also can exist as a long filament [74]. The virus particle, or virion, is composed of a capsid that contains the virus genome, where the capsid itself is encased by a lipid envelope (Figure 2.1). The surface of the particle is studded with protein spikes called hemagglutinin (HA) and neuraminidase (NA). Influenza A viruses are usually referred to by their subtype, e.g., A/H1N1. There is a diverse array of HA (17 subtypes, numbered H1 through to H17) and NA (nine subtypes, numbered N1 through to N9) [112]. Influenza epidemics in humans are usually caused by HA subtypes H1, H2, H3, and NA subtypes N1, N2. For example, seasonal influenza A virus strains of subtypes H1 and H3 circulated in the 2016-2017 flu season in Canada [88].

Generally, the HA and NA are important because they are recognized as antigens by the immune response in a host. The HA and NA also change over time, under selective pressure from the immune system, as the virus accumulates point mutations in the genes for $\mathrm{HA}$ and NA in a process called antigenic drift. In this work, the antigenicity and 


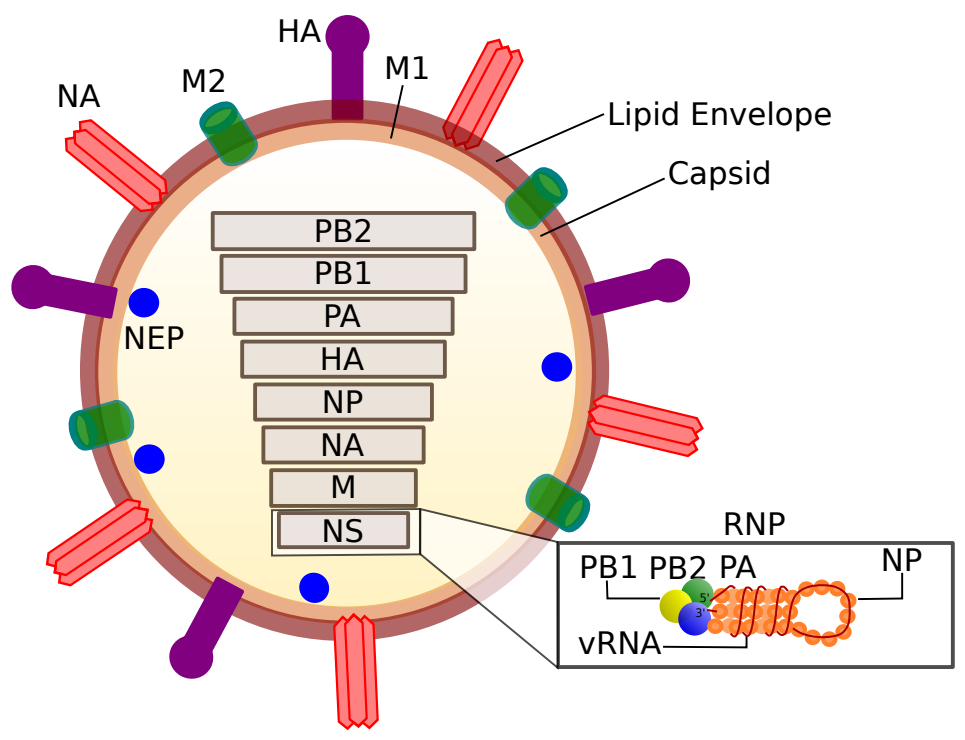

Figure 2.1: Structure of influenza A virion. The influenza A virus is a spherical particle with a lipid envelope encasing a capsid made of M1 protein. Embedded in the surface of the particle are viral proteins HA, HA, and M2 proteins. Contained within the particle are eight vRNA gene segments, which are encapsidated by NP and exist as rod-shaped vRNP (inset). Each vRNP is associated with viral polymerase that is composed of three protein subunits PB1, PB2, and PA. [Adapted from Schwendener Forkel, 2016 [22].] 
immunogenicity of HA and NA are not discussed further since we focus on infections in vitro where immune responses are absent. Otherwise, the functional balance between HA and NA is important because it determines virus fitness and transmissibility [29, 31]. In particular, HA and NA are significant to this work when we study their roles in virus release and the spread of infection in vitro.

The capsid of the virion is formed from matrix protein (M1) and is also studded with proton channels called the matrix-2 protein (M2). Within the capsid, eight negativesense, single-stranded segments of viral RNA (vRNA) are held in place by M1. The gene segments encode for at least one viral protein and can be referred to as the PB2 (polymerase basic 2), PB1 (polymerase basic 1), PA (polymerase acid), HA, NP (nucleoprotein), NA, M, NS (nonstructural) segments (listed here in decreasing length). The M segment encodes for both M1 and M2 proteins, while the NS segment encodes for NS1 (nonstructural 1) and NEP/NS2 (nuclear export/nonstructural 2) proteins. The vRNA segments are present as rod-shaped ribonucleoprotein complexes (vRNP, reviewed in [113]), depicted in the inset of Figure 2.1. In each vRNP, the vRNA is encapsidated with nucleoprotein (NP) (24 nucleotides (nts) of RNA per NP) and twisted into a double helix [90]. One end of the vRNP forms a loop, and the other end where the vRNA termini complement each other and meet, is associated with RNA-dependent RNA polymerase. The viral polymerase itself is composed of three viral protein subunits PB1, PB2, PA.

The segmented nature of the influenza A virus genome drives reassortment, another evolutionary force. If different viruses infect the same cell, a new virus can emerge that possesses a genome containing a mix of different parent segments. In general, this is important because it drives antigenic shift, the process where a novel strain of virus emerges with a new HA and NA subtype combination on its surface. Compared to the slow and continuous process of antigenic drift, antigenic shift introduces abrupt changes to the virus genome and is responsible for the appearance of pandemic viruses. In this work, however, the segmented nature of the influenza virus genome is important in understanding the generation of defective interfering particles and how they parasitize the virus replication cycle.

\subsubsection{Virus replication cycle}

The influenza A virus replicates within cells and its life cycle is depicted in Figure 2.2. Virus entry is the first step in the cycle. The virion attaches to the cell by using HA to bind sialic acid receptors that are present on the cell surface (Step 1). The bound virion initiates endocytosis through a variety of pathways [59], e.g., clathrin-mediated endocytosis, and the endosome is transported towards the nucleus. Along the way, the 
endosome undergoes a drop in $\mathrm{pH}$ caused by the M2 proton channel. Acidification triggers a conformational change in HA that leads to fusion between the lipid membrane of the virion and the endosome [47], and also dissociates the vRNPs from M1 (Step 2). Membrane fusion allows for the virion to uncoat, releasing the vRNPs into the cytoplasm for nuclear import (NP possesses a nuclear localization signal; NLS) [113].

Once inside the nucleus, each vRNP acts independently of each other to carry out transcription and replication. Transcription of vRNA into positive-sense mRNA is performed by the parent viral polymerase (i.e., the one attached to the vRNP), but requires primer-dependent initiation (Step 3). The primer is obtained in a process called cap snatching where the viral polymerase must bind to the host cell's polymerase to obtain a small snippet (10-13 nts long) of the host cell pre-mRNA. Upon initiation, the parent viral polymerase traverses the vRNA template to synthesize mRNA in cis [51] (Figure 2.3, top). The parent viral polymerase eventually encounters steric hindrance towards the end of the template, causing it to stutter repeatedly over a stretch of poly(U) and synthesize a long poly(A) tail on the mRNA transcript. The final mRNA transcript is an incomplete copy of the vRNA segment, that is protected from degradation by a cap at its start and by a poly (A) tail at its end. At the end of primary transcription, the mRNA transcripts are shuttled out of the nucleus for translation and protein synthesis to occur (Step 4). The viral surface proteins HA, NA, M2 are transported to the cell surface (Step 5a), while other proteins such as NP, M1, NS1, NEP, and the polymerase subunits are directed into the nucleus (Step 5b).

In addition to transcription, the same incoming vRNPs are involved in a two-stage replication process (Step 6). In the first stage of replication (incoming (-)vRNA to $(+) \mathrm{cRNA}$ ), positive-sense cRNA is synthesized from the $\mathrm{vRNP}$ where initiation is primerindependent. A complete complementary copy of the vRNA segment is produced and must be stabilized in the form of cRNP. It is unknown how the parent viral polymerase copies the vRNA template without early termination, as in transcription. There is much debate over what causes the transition between transcription and replication [87, 90]. One hypothesis proposes that there is some viral product (e.g., NP [21, 77], small virion RNAs [84]) made during protein synthesis that causes a switch from incoming vRNPs exclusively synthesizing mRNA early on, to synthesizing cRNA later in the life cycle (see [53] for the kinetics of the three types of viral RNA). Another hypothesis proposes that both mRNA and cRNA are stochastically synthesized from the incoming vRNP [104, 105], however any cRNA made early on will degrade until de novo viral polymerase and de novo NP become available to stabilize it in the form of cRNP. The cRNA is stabilized by first binding to a viral polymerase, followed by NP oligomeriza- 


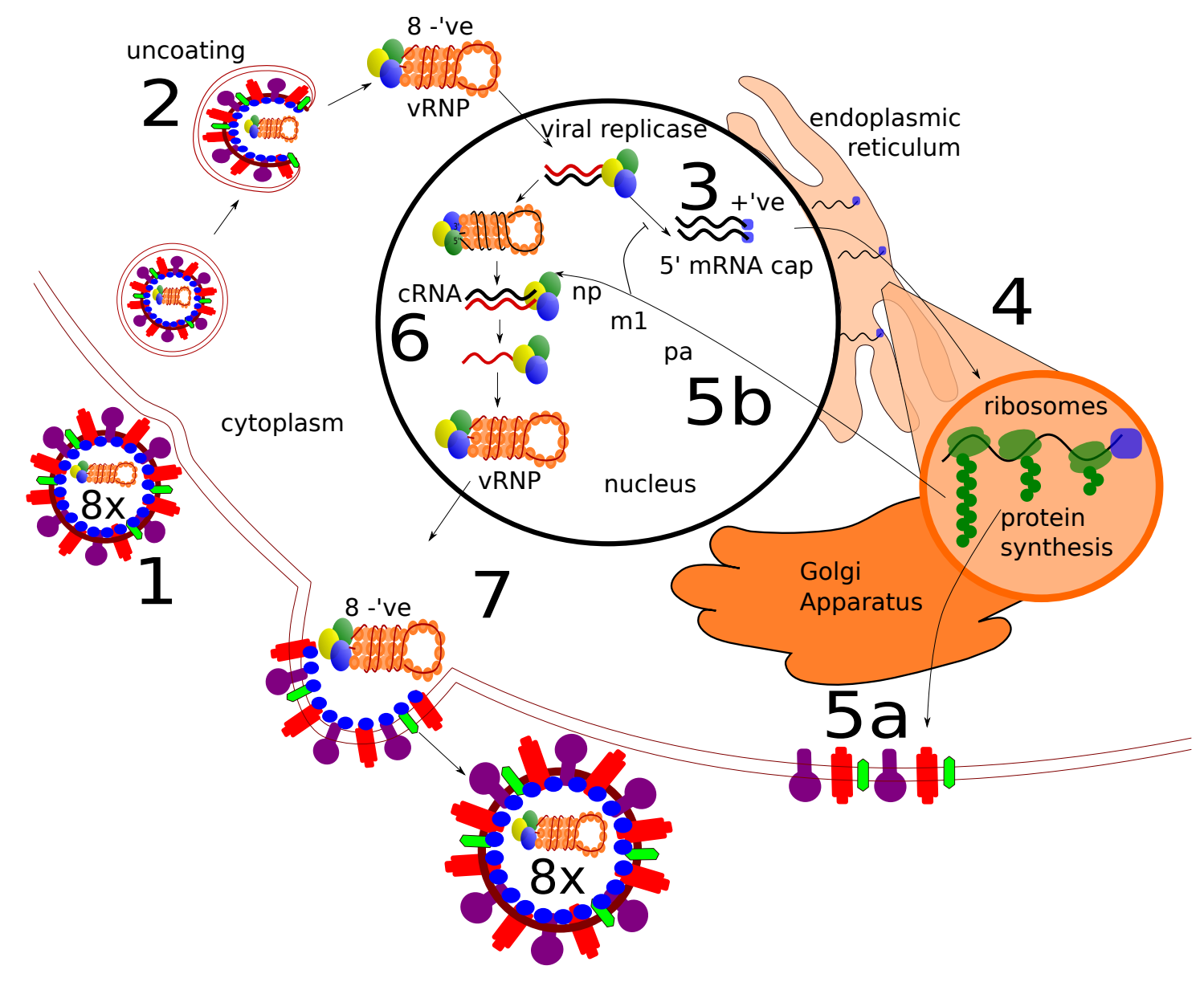

Figure 2.2: Influenza A virus replication cycle. The influenza A virus replicates by first binding to a cell and initiating virus entry via endocytosis (Step 1). As the endosome moves towards the nucleus, the endosome acidifies and fuses with the virus particle, uncoating its genetic material (Step 2). The vRNPs are imported to the nucleus where primary transcription occurs to produce mRNA (Step 3). Copies of mRNA are shuttled out of the nucleus for translation and viral protein synthesis to take place (Step 4). Some viral proteins are transported to the cell surface (Step 5a), while others are imported to the nucleus to facilitate viral replication (Step 5b). During viral replication, cRNA are produced from vRNPs, and stabilized into cRNPs. Progeny vRNA, stablilized as vRNP, are produced from cRNPs (Step 6). As viral replication shuts down and nuclear export of progeny vRNP occurs, eight progeny vRNPs assemble underneath the cell membrane to be packaged and budded into progeny virion (Step 7). In the final step, NA must cleave the budded virion from the cell surface to successfully release a new virus particle. [Adapted from Schwendener Forkel, 2016 [22].] 


\section{Transcription ( $v R N A$ to mRNA)}
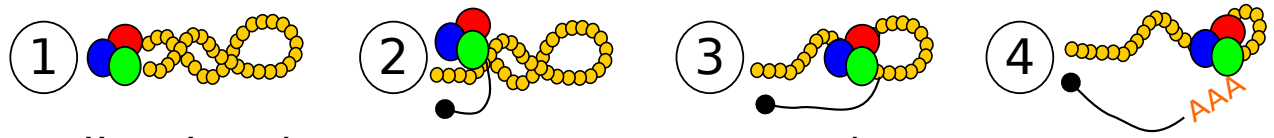

Replication (cRNA to progeny $v R N A)$

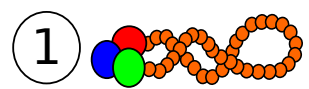

(4)

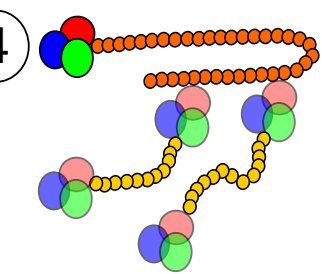

(2)
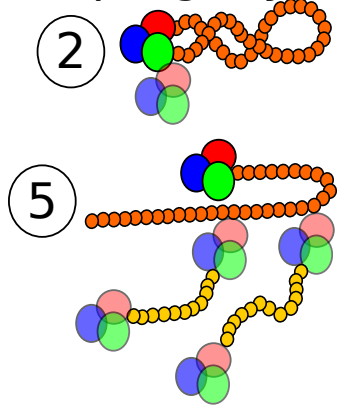

(3)

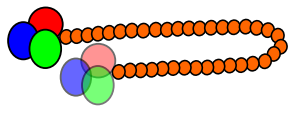

(6)

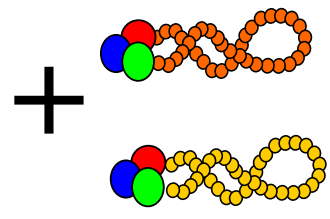

viral polymerase

- mRNA primer c0000 VRNA template (-)

\section{progeny polymerase} AAA amino acids œ cocos cRNA template $(+)$

Figure 2.3: Viral polymerase acts as both transcriptase and replicase. (Top) During transcription, the viral polymerase associated with the vRNP synthesizes mRNA in cis. After obtaining a mRNA primer from the host cell (cap snatching), mRNA synthesis proceeds with the viral polymerase traversing the template vRNA strand until it encounters steric hindrance. The viral polymerase stutters over a stretch of the template, synthesizing a long poly (A) tail on the mRNA transcript, before termination. (Bottom) During replication, de novo (progeny) viral polymerases initiate synthesis of vRNA in trans, using cRNA as the template strand. Progeny vRNA must be stabilized by de novo polymerase and encapsidated by NP to form vRNP. [Adapted from Schwendener Forkel, $2016[22]$. 
tion, to form cRNP. In the second stage of replication $((+)$ cRNA to progeny (-)vRNA), de novo viral polymerases synthesize negative-sense vRNA from cRNPs in trans [51] (Figure 2.3, bottom). The resultant vRNA form progeny vRNP for the eventual incorporation into progeny virion. Still within the nucleus, the progeny vRNPs amplify rounds of (secondary) transcription and replication until the build-up of M1 initiates the nuclear export of vRNPs, coincident with the shutdown of replication (inhibition of synthesis of positive-sense RNA). To facilitate nuclear export, M1:NEP complexes bind to progeny vRNPs, masking the NLS [113]. The NEP itself possesses a nuclear export signal.

Outside the nucleus, the progeny vRNPs and M1 assemble underneath, and orthogonal to, the membrane which has viral surface proteins (HA, NA, M2) embedded in it [78]. Eight progeny vRNPs are selectively packaged into a ' $7+1$ ' configuration (seven vRNPs surrounding a center vRNP), where each one is linked to another [113]. The vRNPs push outwards forming a bud [74] (Step 7). When bud growth and closure are complete, the budded virion remains bound to the cell surface since HA also binds cell receptors upon exit [97]. In the final step of the viral life cycle, NA must cleave the virus-cell attachments in order to release progeny virion.

\subsubsection{Antiviral therapies}

Here, we briefly summarize the modes of action of antivirals used in the suppression of influenza A virus infection. In Figure 2.4, three classes of antivirals and their inhibition of key steps in the viral replication cycle are shown. One class of antivirals is the fusion inhibitors, which include amantadine and rimantadine. These antivirals block the M2 proton channel, thereby preventing acidification and uncoating of the virion. Since vRNA is not imported to the nucleus, viral replication and transcription cannot proceed. Another class of antivirals is the viral polymerase inhibitors, such as favipiravir and ribavarin. These mimic nucleotides, resulting in the viral polymerase misincorporating the nucleoside analogue during RNA synthesis. Such a mechanism exploits RNA polymerases' lack of proofreading ability and increases the number of errors introduced to copies of the virus gene segments. Thus, errors accumulate during replication and transcription, and viral proteins may also be misfolded. Lastly, are the neuraminidase inhibitors (NAIs) which include zanamivir, oseltamivir, peramivir, and laninamivir. These were designed to mimic sialic acid and to bind to the active site of NA, thereby inhibiting its action. As such, NAIs prevent the release of progeny virion. Both viral polymerase inhibitors and NAIs become the subject of Chapter 4 


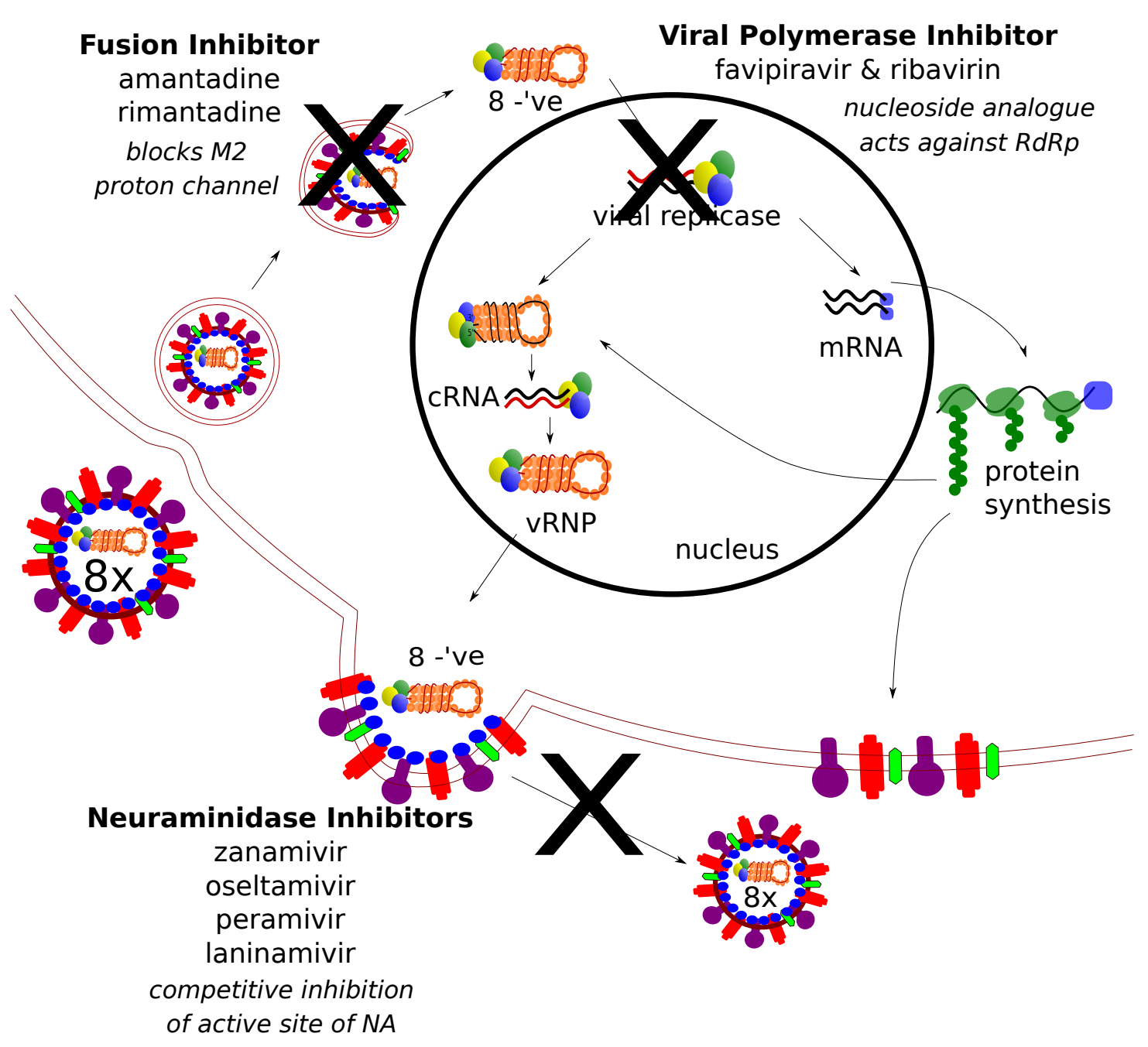

Figure 2.4: Modes of action of antivirals. Antivirals inhibit a variety of steps in the influenza A virus replication cycle. Fusion inhibitors block virus fusion and uncoating, preventing the virion from ejecting its genetic material into the cell. Viral polymerase inhibitors act as nucleoside analogues to interfere with the synthesis of RNA, impairing transcription and replication. Neuraminidase inhibitors bind to the active site of NA, preventing NA from cleaving budded virion, thereby inhibiting the release of progeny virus. [Adapted from Schwendener Forkel, 2016 [22].] 


\subsection{Previous mathematical models}

In this section, we survey previous MMs that have been used to study DIPs, and MMs that have been used to study influenza A virus release.

\section{Mathematical models of infection with DIPs in vitro and in vivo}

There have been MMs developed for the study of DIPs within in vitro infections and in vivo infections. Here, we consider MMs that give a mechanistic description of viral infection in animal cells (i.e., excluding bacteriophages).

Previous MMs of in vitro infections with DIPs suffer from one major disadvantage - they describe generic virus-cell interactions, instead of representing a specific virus (e.g., influenza A virus) infecting a specific cell type (e.g., Madin Darby canine kidney cells; MDCK). For example, Kirkwood and Bangham were able to account for the effect of DIPs on standard (i.e., infectious and non-defective) virus, without invoking external factors such as interferon [55]. The MM was able to qualitatively reproduce in vitro phenomena such as persistent infection, oscillations in virus titers during passaging, and the sporadic disappearance of virus and DIPs (i.e., "self-curing" of infections) after repeated passages. The MM by Kirkwood and Bangham is a set of multicompartment ordinary differential equations (ODEs) that informs our MM in Chapter 3. However, our MM quantitatively describes infection with influenza A virus and DIPs. One other in vitro MM subsequently built upon the Kirkwood and Bangham MM, by incorporating spatial dimensions and the diffusion of standard virus and DIPs [23]. Again, the MM was generic and did not represent specific virus-cell interactions.

Only recently have there been MMs specifically studying influenza A DIPs in a quantitative way. In the work by Frensing et al., the MM describes populations of standard virus, DIPs, and cells cultivated as a continuous infection inside a bioreactor [25]. Frensing et al. were interested in achieving a continuous production of virus for the manufacture of vaccines, but high virus yields were consistently hampered by the generation and accumulation of DIPs. With their MM, they were able to recapitulate the periodic oscillations observed in the virus titer data from the bioreactor, that was consistent with the presence of DIPs. They tested whether DIP formation could be eliminated in the MM by reducing two parameters: a) the amount of DIPs present in the infecting virus stock (which is believed to be minimized through low MOI passaging), and b) the rate of de novo generation of DIPs (possibly controlled through optimized cell lines or virus strains). In the end, they concluded that a continuous infection that is free from the suppressive effects of DIPs is unlikely to be achieved, since it is not possible to fulfill two 
conditions: that the virus stock be made perfectly DIP-free and the rate of de novo generation of DIPs be zero. In our view, Frensing's MM is simplistic because it ignores the effects of the co-infection window and superinfection exclusion (i.e., cells infected with virus will prevent co-infection by DIPs partway through the replication cycle), which our $\mathrm{MM}$ in Chapter 3 accounts for.

Another recent MM describes intracellular replication of influenza A virus, and explores a mechanism of DIP interference [60]. The MM implements a replication advantage for DI RNA segments, compared to full-length segments of RNA. With this, the MM predicts that the main interference mechanism is the competition for viral resources, specifically the depletion of NP. The authors also go on to propose several hypotheses regarding DIPs, e.g., explaining the predominance of DI RNA originating from the segment encoding for polymerase, which have yet to be supported by experimental measurements. Such an MM was limited to describing a single cell, rather than a population of cells as in our MMs in Chapters 3 and 4.

For completeness, we also briefly summarize MMs of DIP infection dynamics in vivo. While past in vitro MMs were generic and not specific to any virus (until recently in the last 5 years), in contrast, in vivo MMs have been predominantly developed for the study of human immunodeficiency virus (HIV). The first in vivo MM of DIPs was studied in the context of HIV therapy [76]. Following this, a study theoretically explored the possibility that DIPs can exacerbate an HIV infection in vivo [7]. It was demonstrated that there exist two conditions in which DIPs may increase the total HIV growth rate: DIPs must be able to escape immune detection, yet still impair the immune response. Another MM analyzed HIV superinfection where the defective mutant can insert its genome, but not transcribe due to a lack of viral proteins [69]. The MM dynamics were driven by superinfection, immune escape of DIP, and host cell limitations. Most recently, an intracellular MM of DIP interference was used in conjunction with an in vivo MM in [92]. Here, they were concerned with testing evolutionary stable DIP interference mechanisms (i.e., genome stealing vs. capsid stealing). It is not clear how the study of DIPs of other viruses might translate into findings that are applicable to the study of influenza A DIPs, because of the different mechanisms of interference involved.

\section{Mathematical models of influenza A virus release}

To our knowledge, there has only been one MM of intracellular influenza A virus replication that has explicitly represented virus release (i.e., NA cleaving virus-cell attachments of budded virion) by Schwendener Forkel et al. [22]. Such a MM was based on a more complex MM by Heldt et al. [34]. In the work by Heldt et al., however, an explicit virus 
release rate was not used. Instead, their "release" rate actually represented the combined rates of assembly, budding, and release. The main result of Schwendener Forkel et al.'s work was the development of a reduced version of the Heldt MM, with 23 fewer parameters and 19 fewer variables, that performed equally as well. The MM by Schwendener Forkel et al. was made to be as simple as possible, but no simpler.

To calibrate the MMs, Schwendener Forkel et al. fitted their reduced MM and the Heldt MM to intracellular influenza A virus kinetics data such as time courses of the percent of virion fused during viral entry [100], and copies of mRNA, cRNA, and vRNA [53]. The resultant best fit value of the explicit virus release rate in the Schwendener Forkel MM was $1.5 \mathrm{~h}^{-1}$. Once both MMs were able to reproduce the data, the MM predictions were tested. Both MMs were used to simulate a single-cycle growth in vitro assay, and it was found that the predicted extracellular virus kinetics from either MM was able to match the infectious virus titers measured for the H1N1pdm09 WT-I223V virus strain in [81]. Following this, only the predictions from Schwendener Forkel MM were further tested by simulating infections in the presence of antivirals. Consistent with experimental observations, the reduced MM predicted that polymerase inhibitors are much more effective at suppressing extracellular virus growth than NAIs or fusion inhibitors. However, the reduced MM also made an incorrect prediction about the extracellular virus under therapy with NAIs or fusion inhibitors - namely that extracellular virus is not at all reduced by such antivirals, though a delay in the virus peak can be observed. Given that the MM by Schwendener Forkel et al. was not fitted to extracellular virus data, and that the resultant predictions failed to capture the correct behaviour under NAI therapy, one must be careful not to overinterpret the value of the explicit release rate obtained.

Our MM in Section 4.4 is different than the Schwendener Forkel MM because of the scale of infection that is represented (infection in a population of cells vs. inside a single cell), but presents a complementary view of infection. In our MM and the Schwendener Forkel MM, virus release is explicitly represented in the same way, such that results from either MM can be used to inform the other. 


\section{Chapter 3}

\section{(In) validating}

experimentally-derived knowledge about influenza A defective interfering particles

This chapter is based on the following published paper:

L. E. Liao, S. Iwami, and C. A. A. Beauchemin. (In)validating experimentally derived knowledge about influenza A defective interfering particles. J R Soc Interface. 2016;13(124):piiL20160412. doi:10.1098/rsif.2016.0412. 


\begin{abstract}
A defective interfering particle (DIP) in the context of influenza A virus is a virion with a significantly shortened RNA segment substituting one of eight full-length parent RNA segments, such that it is preferentially amplified. Hence, a cell co-infected with DIPs will produce mainly DIPs, suppressing infectious virus yields, and affecting infection kinetics. Unfortunately, the quantification of DIPs contained in a sample is difficult since they are indistinguishable from standard virus (STV). Using a mathematical model, we investigated the standard experimental method for counting DIPs based on the reduction in STV yield (Bellett \& Cooper, 1959). We found the method is valid for counting DIPs provided that: (1) a STV-infected cell's co-infection window is approximately half its eclipse phase (it blocks infection by other virions before it begins producing progeny virions); (2) a cell co-infected by STV and DIP produces less than 1 STV per 1,000 DIPs; and (3) a high MOI of STV stock ( $>4$ PFU/cell) is added to perform the assay. Prior work makes no mention of these criteria such that the method has been applied incorrectly in several publications discussed herein. We determined influenza A virus meets these criteria, making the method suitable for counting influenza A DIPs.
\end{abstract}

\title{
3.1 Introduction
}

A cell that is infected with standard virus, will produce progeny that is a mixture of infectious, functional virus - hereafter referred to as standard virus (STV) - and particles that are defective (figure 3.1, top row) since viral replication is an error-prone process, especially for RNA viruses. Defective, virus-like particles that either cannot initiate infection or can but are replication-incompetent, are referred to as defective particles. If these defective particles do not interfere with STV replication, they are called defective non-interfering particles [10] (figure 3.1, middle row). In contrast, defective particles that can initiate infection, but are replication-incompetent in a manner that causes them to interfere with STV replication, are known as defective interfering particles (DIPs) (figure 3.1, bottom row). The properties of DIPs have been enumerated in [42]. Namely, they resemble STV since they are composed of the same structural proteins, they contain an incomplete copy of the viral genome, and they require STV to replicate.

Owing to differences in the mechanisms of infection and replication of different viruses, what constitutes a DIP, i.e. the factors that can cause a defective particle to interfere with the replication of its STV counterpart, will vary across viruses. Herein, our results and analyses focus on DIPs in the context of the influenza A virus. Influenza A virions have a segmented genome comprising 8 negative-sense viral RNA (vRNA) segments packed within a capsid, enveloped by a host membrane containing embedded 


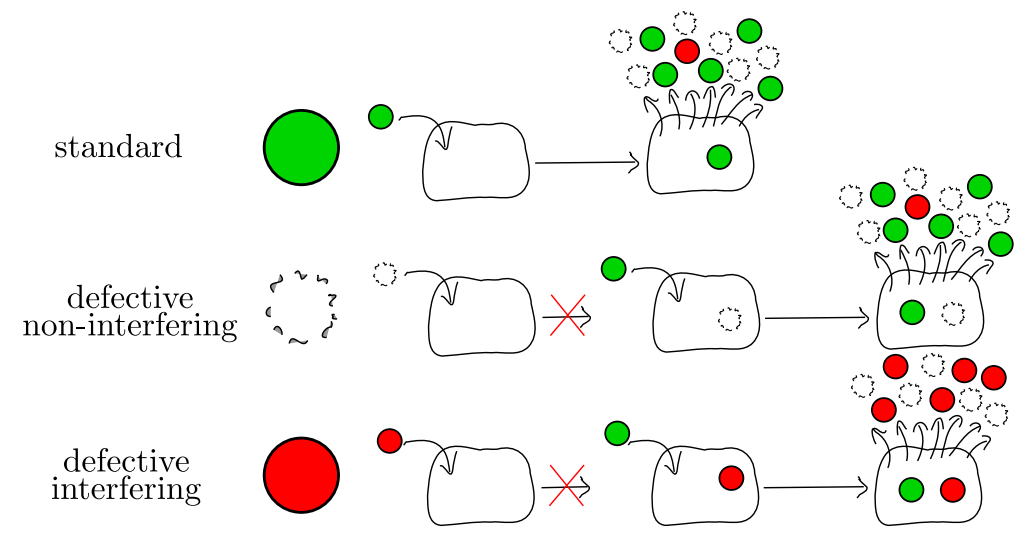

Figure 3.1: Process of standard virus yield suppression by DIPs. (Top row) A cell infected by standard (non-defective) influenza A virus (STV) yields virus progeny comprising STV, DIPs, and defective non-interfering particles. (Middle row) Defective non-interfering particles are either unable to enter susceptible cells, or can enter cells but are replication-incompetent in a manner which does not interfere with further infection by STV. (Bottom row) In contrast, DIPs can infect cells, but only upon additional infection with STV can the co-infected cell produce progeny, though DIPs will be produced at the expense of STV in these cells, resulting in suppression of STV yield.

viral proteins. An influenza A DIP is identical to its STV counterpart, however, at least one of its 8 vRNA segments have been replaced by a defective interfering (DI) vRNA segment (for a review, refer to [15]). When interference has been observed, influenza A DI vRNAs were shown to contain a large internal deletion making them shorter than their corresponding full-length parent segment [13]. Influenza A DI vRNAs originating from vRNA segments that encode for viral polymerase (PB2, PB1, and/or PA) have been observed most frequently $[13,14]$, though influenza A DI vRNAs originating from other segments have also been observed [49].

On short time scales, the dynamics of DIPs are governed by two processes shown in figure 3.1: de novo generation (top row) and their amplification (bottom row). A cell infected only by an influenza A DIP with a truncated polymerase vRNA segment cannot produce de novo functional viral polymerase, such that the virus replication cycle cannot be completed and will not yield any virus progeny. Upon co-infection with influenza A STV, the full-length polymerase vRNA segment contributed by the STV will be translated into functional viral polymerase which will proceed to replicate and transcribe all segments contributed by the DIP, including its shortened, DI polymerase segment, thereby successfully completing the virus replication cycle to produce progeny. 
It is hypothesized that more copies of the shorter influenza A DI segment are produced per unit time than the full-length segment, and that the greater number of DI segments sequesters intracellular resources, inhibiting STV replication [66]. As reviewed in [24], there are still many open questions about the mechanism of influenza A DIP interference, and the length advantage of influenza A DI vRNA is not the sole explanation for interference. For example, it has been shown that influenza A DI vRNAs are packaged more efficiently than standard vRNA $[19,80]$. Ultimately, a cell co-infected by influenza A DIPs and STV will produce mainly progeny DIPs at the expense of STV, resulting in significant suppression of influenza A STV yield.

Since the suppression of influenza A STV yield by DIPs requires the co-infection of a cell by both a STV and a DIP, the effect of DIPs is thought to be significant only in assays where the likelihood of a co-infection event is high. An experiment conducted at low multiplicity of infection (MOI), e.g., with an inoculum containing one virion per 100,000 cells, is unlikely to result in any one cell receiving two or more virions. Inoculation at a high MOI, e.g., 4 plaque forming units (PFU) per cell, however, will result in a large number of co-infected cells. This is why the presence of DIPs in an inoculum of high MOI causes a significant reduction in STV yield compared to one of low MOI. This can be seen in figure 3.2 where an influenza A virus infection conducted with a high MOI resulted in a 5,000-fold reduction in peak STV concentration but the same total (STV + defective) particle peak concentration compared to that observed for the same experiment conducted at a low MOI. Others have also observed a reduction in influenza A STV yield due to DIPs, with no decrease in the total influenza A particles produced $[52,75]$.

Even in an assay initiated with a highly diluted inoculum infecting only a few cells, a high rate of virion production by these cells might, at least transiently, lead to a high virus concentration in their immediate vicinity. This high local concentration could, theoretically, result in the co-infection of at least some cells. Therefore, the presence of DIPs in a sample, even in assays inoculated with a low MOI, has the potential to impact conclusions drawn from measurements of STV. For example, when comparing the virulence of strains A and B, observing lower STV yield for strain A would be thought to indicate a lower virulence. On the other hand, the lower STV yield could be due to the presence of a large proportion of DIPs in the sample for strain A which would otherwise exhibit STV yields similar to, or even greater than, that of strain B. To avoid such confounding effects, it is thought that passaging virus samples at low MOI, especially in combination with plaque purification $[24,26]$, can reduce the proportion of DIPs in samples to sufficiently low levels so as not to interfere with common assays. 

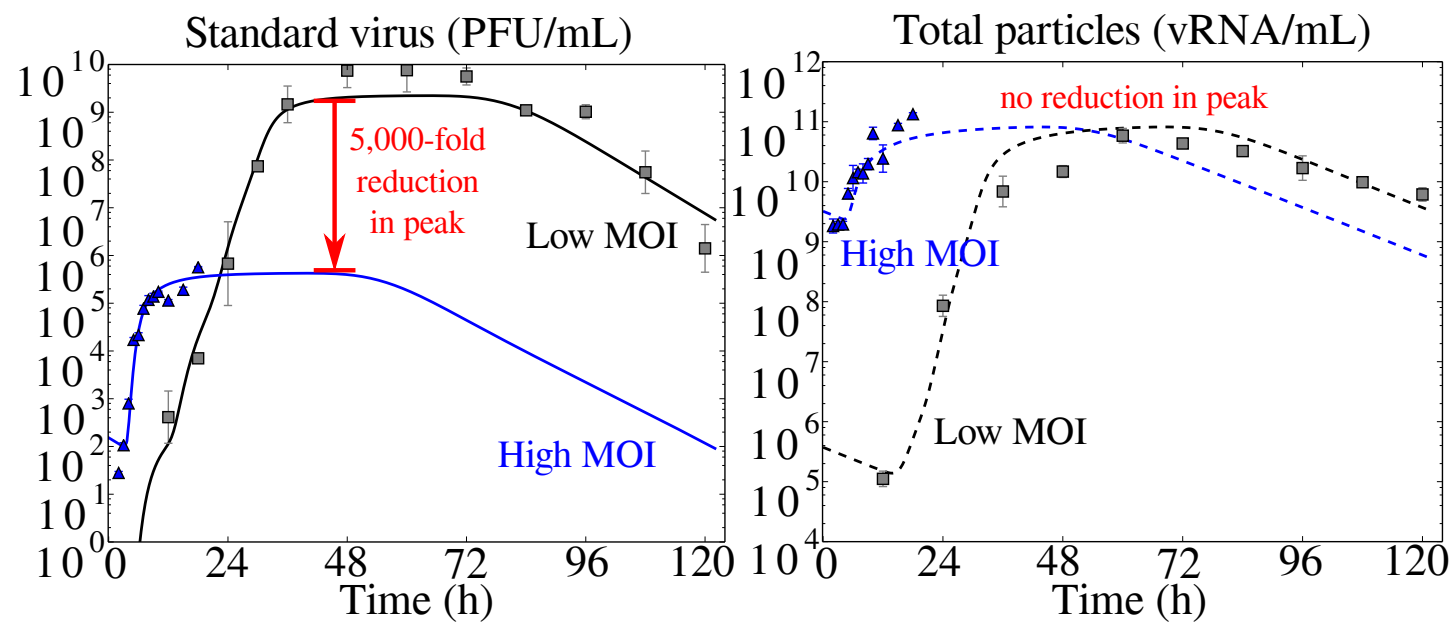

Figure 3.2: The presence of DIPs is only evident at high STV MOI. The concentration of standard infectious virus (STV; Left) and total (STV + DIP + defective noninterfering) particles (Right) were measured at various time points over the course of two in vitro infection experiments with the 2009 pandemic influenza A/Québec/144147/09 (H1N1) virus strain. The curves are the result of a previously reported mathematical analysis of these infections [85]. Both experiments were performed at the same time, in the same manner, differing only in the concentration of STV in the initial inoculum, with one conducted at a low MOI of $10^{-5} \mathrm{PFU} /$ cell (grey), and the other at a high MOI of $4 \mathrm{PFU} /$ cell (blue). While the peak total particles for both infections was $10^{11} \mathrm{vRNA} / \mathrm{mL}$ (Right), a 5,000-fold drop in the STV peak is observed (Left) for infection with a high MOI. It is typically assumed that this reduction in STV yield is evidence of the presence of defective interfering particles (DIPs) in the STV stock. 
Influenza A DIPs differ from their STV counterpart only in a deletion in one of the 8 influenza A vRNA segments, which are packed into the same capsid, enveloped by the same host membrane, with the same embedded proteins. This minor difference between influenza A DIPs and STV is insufficient to allow for their separation and quantification based on physical characteristics (e.g., appearance, weight, volume, charge), an issue common to other viruses as well [41, 64, 73, 108, 110]. For this reason, in 1959, Bellett and Cooper (hereafter, B\&C) introduced an assay whose data can be used to compute, rather than directly measure, the content of DIPs in a sample by indirectly inferring their concentration based on the observed reduction in STV yield they cause [6]. To this day, the B\&C assay and variations thereof which rely on the same principles and assumptions, continue to be the primary manner by which DIPs are quantified [65, 95, 114]. Much of what is thought to be known about DIPs and the methodologies developed to mitigate their impact, are based on inferences drawn from these indirect quantifications. Unfortunately, when performing different variations of the B\&C assay, others have commonly observed deviations of their experimental data from the theoretical trend predicted by the $\mathrm{B} \& \mathrm{C}$ method $[6,64,65,86,103,109]$. This indicates that, at least under some conditions, the $\mathrm{B} \& \mathrm{C}$ calculation is invalid and its use could lead to incorrect conclusions about the presence of DIPs in a virus sample, or misguided inferences about their impact under certain assay conditions.

In the present work, we revisit the B\&C assay in the context of influenza A DIPs. We evaluate the extent of its validity using a mathematical model for influenza A virus infection that explicitly accounts for DIPs. We identify the assumptions made by B\&C in computing the concentration of DIPs based on the reduction in infectious STV yield. We establish conditions under which the B\&C assay must be performed for these assumptions to hold true. We also provide explanations and possible remedies for deviations of experimental observations in the $\mathrm{B} \& \mathrm{C}$ assay from the trend predicted theoretically by the $\mathrm{B} \& \mathrm{C}$ calculation. 


\section{$3.2 \quad$ Results}

\subsubsection{Theoretical basis and failure of the Bellett \& Cooper assay}

In 1959, Bellett \& Cooper performed an assay, which exploits the reduction of STV yield observed in the presence of DIPs, to determine the concentration of DIPs in a sample of vesicular stomatitis virus (VSV). They infected parallel cell cultures with an inoculum consisting of a known, fixed concentration of a pure, DIP-free standard VSV stock, mixed with increasing dilutions of a sample containing an unknown concentration of standard VSV and DIPs. The results of this important experiment are shown in figure 3.3, where a marked decrease in the STV yield can be seen for increasing concentrations of their DIP-containing VSV sample. From these data, the authors conclude that, at their chosen volume of inoculum per cell, their undiluted sample contained DIPs at a MOI of $4 \mathrm{DIP} /$ cell. Their conclusion relies on a number of simple, but seemingly reasonable steps.

As illustrated in figure 3.3, given a particular MOI of DIP and STV per cell, the distribution of these particles within a population of cells is statistically described by the Poisson distribution. Hence, from the number of each type of infecting particle (STV, DIP) it is possible to theoretically calculate the fraction of cells infected only with DIPs or STV, co-infected by both, or uninfected (For this calculation, see Appendix Section A5). For example, inoculating cells with $4 \mathrm{PFU} /$ cell and no DIPs will result in $98 \%$ of cells infected by STV alone, while inoculating with $4 \mathrm{PFU} /$ cell $+1 \mathrm{DIP} /$ cell will result in $36 \%$ STV-only infected cells, or $37 \%\left(\frac{36 \%}{98 \%} \times 100 \%\right)$ of that in the absence of DIPs. B\&C further assume that the STV yield is proportional to the fraction of STV-only infected cells, i.e., they assume that STV-only infected cells are the sole significant producers of STV. Thus, according to B\&C, the reduction in STV yield corresponds directly to an equal reduction in the number of STV-only infected cells due to co-infection by DIPs. This reasoning was exploited by $\mathrm{B} \& \mathrm{C}$ to relate the STV yield reduction to the reduction in the number of STV-only infected cells, i.e., cells that received one or more STV but received no DIP, and in turn relating that number to the number of DIPs in the infecting inoculum based on the Poisson distribution, e.g., an infectious virus yield that is $37 \%$ of that in the absence of DIPs implies that cells were inoculated at a DIP MOI of 1 DIP/cell.

According to the Poisson distribution, on which B\&C's reasoning relies, the fraction of STV-only infected cells, and thus the STV yield, should decrease exponentially as the number of DIPs in the inoculum is increased. When expressing the relative STV yield on a logarithmic scale versus the linear increase in dose of DIPs received, as in figure 3.3, the data points should fall on a straight line whose slope is equal to the DIP MOI (DIP/cell) 


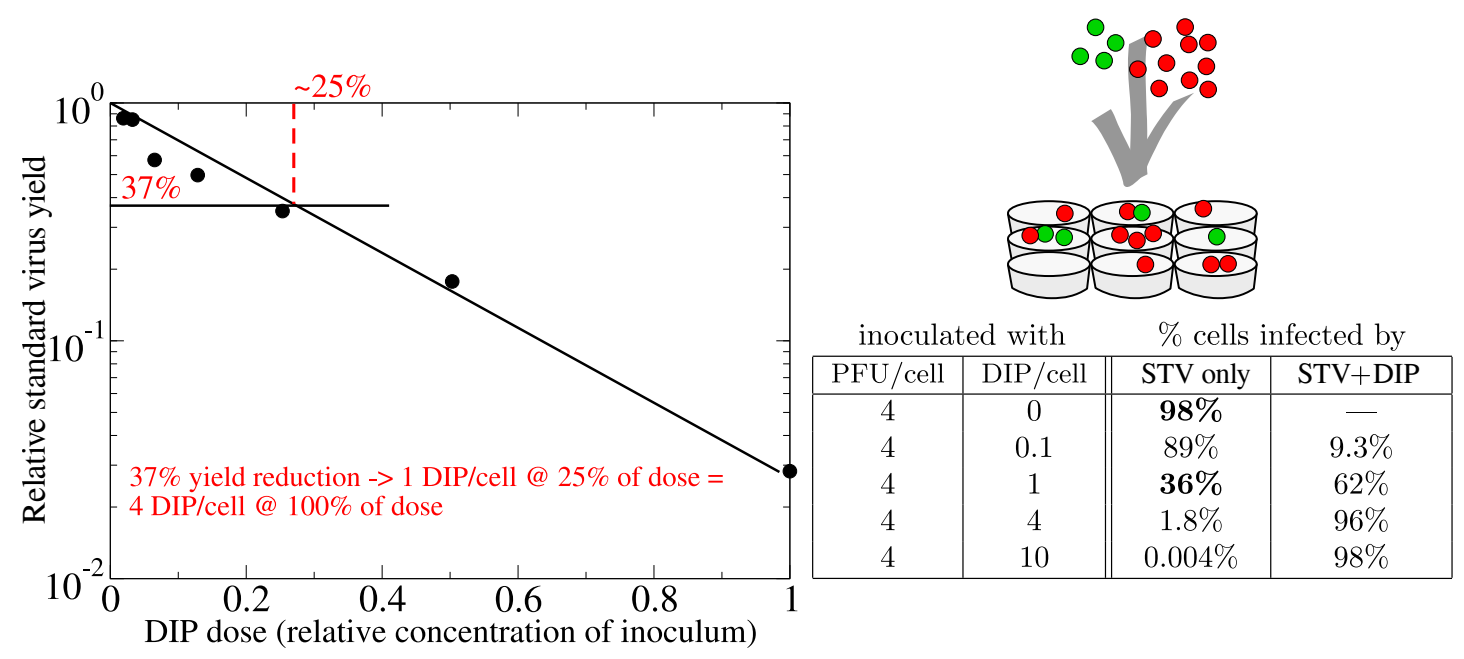

Figure 3.3: Counting of DIPs in the Bellett \& Cooper 1959 assay. (Left) In the B\&C experiment, parallel cell cultures were incubated for one hour with an inoculum containing a fixed, known concentration of a DIP-free standard VSV stock plus increasing dilutions of a VSV sample of unknown DIP concentration, and then rinsed. The STV yield (in PFU/mL) in the cell cultures' supernatant was determined at $20 \mathrm{~h}$ post-rinsing, and expressed relative to the STV yield in the absence of DIPs ( $y$-axis) as a function of decreasing dilutions (increasing DIP doses, $x$-axis) of the virus sample (data taken from figure 1 in [6]). (Right) When inoculating 9 cells with 4 STV (PFU, green) and 10 DIPs (red), a percentage of cells will not be infected while others will be infected by one or more STV and/or DIP. These percentages follow a Poisson process and can be computed (see Appendix A5) for any number of PFUs and DIPs in an inoculum. Assuming that only cells infected by STV alone produce STV, a relative STV yield of $37 \%$ when the sample is diluted in a ratio of $1: 4(25 \%)$ is thought to indicate that the sample DIP MOI was $1 \mathrm{DIP} /$ cell at this dilution, or $4 \mathrm{DIPs} /$ cell when undiluted. 
of the undiluted sample. Thus, having performed the B\&C assay to produce the graph shown in figure 3.3, the DIP MOI of a sample can be determined either directly from the slope of this graph or by taking the reciprocal of the sample dilution factor at which the relative infectious virus yield is $37 \%$, e.g., in figure 3.3 , the inverse of $25 \%$ or $1 / 4=$ $4 \mathrm{DIP} /$ cell.

However, even in B\&C's original publication, the same assay was performed using different DIP-containing VSV samples, in conjunction with varying STV MOIs, and yielded data that did not follow a straight line on a logarithmic-linear plot, as shown in figure 3.4 (left). Although the data clearly disagreed with their theoretical prediction, the authors calculated the undiluted samples' DIP MOI from the reciprocal of the sample dilution factor at $37 \%$ relative STV yield. More recently, Marcus et al. used the B\&C assay to count DIPs in different influenza A virus samples [65]. Again, despite marked deviations of their data from the theoretically predicted trend, Marcus et al. calculated the sample's DIP MOI from the reciprocal of the sample dilution factor corresponding to a $37 \%$ relative STV yield, as shown in figure 3.4 (right). If the experimental data obtained by performing the $\mathrm{B} \& \mathrm{C}$ assay deviate from the theoretically predicted behaviour, is the DIP MOI they yield still accurate? Answering this question requires an understanding of the conditions which give rise to these deviations so as to either avoid them or modify B\&C's assumptions and expand their theoretical framework to account for them.

\subsubsection{A mathematical model of influenza A STV and DIP infection kinetics}

The calculation behind the $\mathrm{B} \& \mathrm{C}$ assay stems from a static view of infection; it overlooks the kinetics of virus attachment, replication, and release, and how these affect a cell's susceptibility to re-infection. To explore these aspects of infection and determine how they can manifest in a $\mathrm{B} \& \mathrm{C}$ assay, we employed a mathematical model for influenza $\mathrm{A}$ STV and DIPs, with emphasis on the details of co-infection. The mathematical model, illustrated in figure 3.5, takes a mesoscopic view of infection, describing only quantities of particles and cells in various states. Hereafter, we will refer to this new mathematical model as the LIB (Liao, Iwami, and Beauchemin) model.

In the LIB model, uninfected cells can be infected by STV. Cells newly infected by STV are not yet able to produce STV for a length of time called the eclipse phase. At the end of the eclipse phase, STV-infected cells produce progeny STV for a length of time called the infectious phase, until the cells die. Uninfected cells can also be infected by DIPs, and enter an arrested state. Arrested cells remain in that state forever, or until they are co-infected by STV. Herein, the term co-infected cell will be used to refer only 

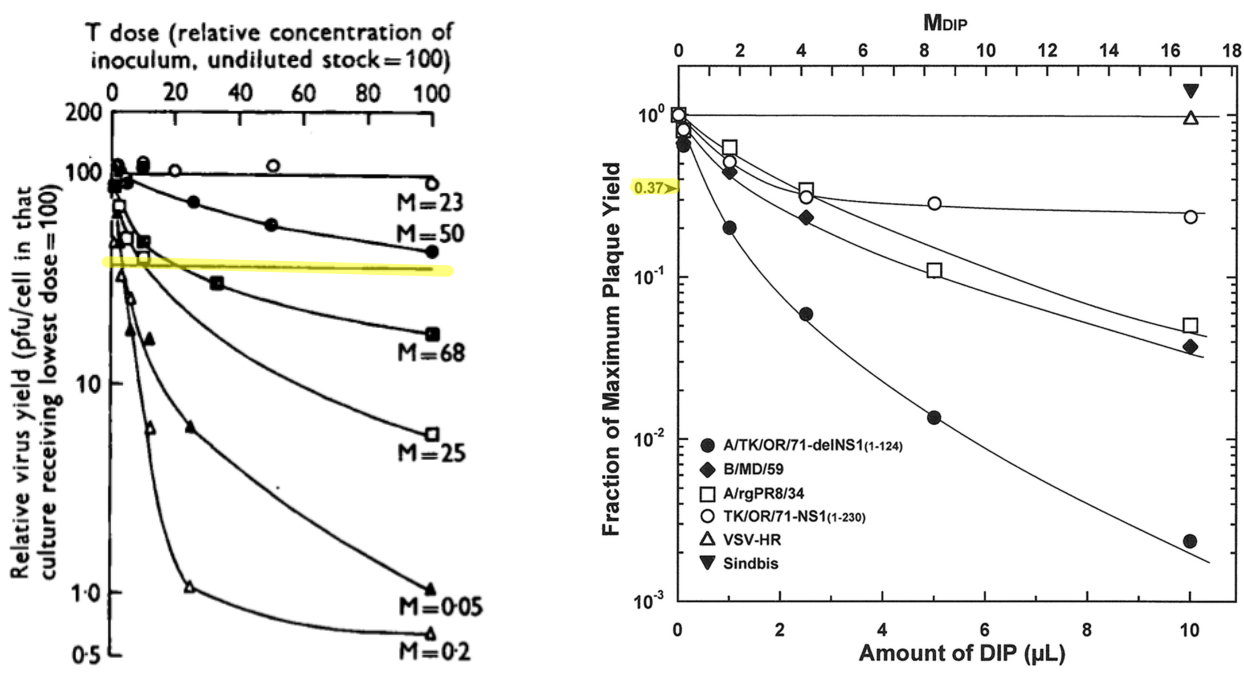

Figure 3.4: Experimental deviation from the theoretical predictions of Bellett \& Cooper. Data do not follow the exponential relationship (which should appear as a straight line on these graphs) theoretically predicted by $\mathrm{B} \& \mathrm{C}$, though this deviation is due to different factors in each case. (Left) B\&C assay applied to VSV where the lines correspond to different STV MOI of the stock DIP-free virus [Reproduced with added highlighting from ref. [6] (Copyright 1959, Microbiology Society)] (Right) B\&C assay applied to quantify DIPs in an influenza A virus sample using different DIP-free virus stocks [Reproduced with permission from ref. [65] (Copyright 2009, American Society for Microbiology)]. Despite this, the authors estimate the DIP MOI of the samples using the reciprocal of the sample concentration corresponding to a $37 \%$ relative infectious virus yield (highlighted). 


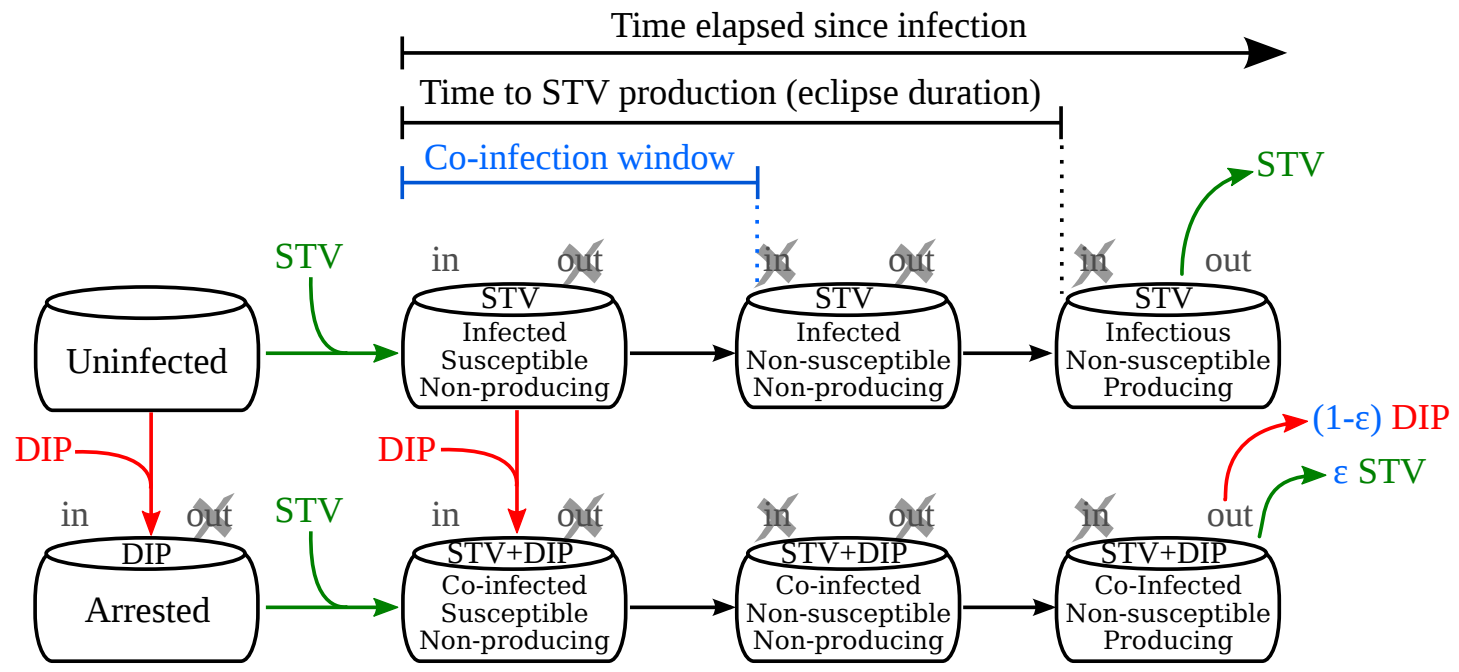

Figure 3.5: Mathematical model for the kinetics of influenza A virus infection in the presence of DIPs. (Top row) The standard branch of infection depicts the progression of a STV-infected cell through an eclipse phase (infected but not yet producing STV) followed by an infectious phase (infected and STV-producing). (Bottom row) The co-infection branch shows a DIP-infected cell in an arrested state, until co-infection by STV which triggers its progression through the eclipse phase, and infectious phase wherein the DIP+STV co-infected cell can produce DIPs, but possibly also STV progeny. Two key aspects of DIP co-infection are highlighted (blue): the fraction of progeny STV produced by co-infected cells $(\varepsilon)$, and the co-infection window, i.e., the time after entering the eclipse phase at which a STV-infected cell ceases to be susceptible to further infection.

to a cell that has been infected by no less than one STV and one DIP, but does not refer to cells super-infected by more than one STV and no DIPs. Like STV-infected cells, cells co-infected with DIP+STV proceed through an eclipse phase during which they produce no STV, and after which they will produce progeny for some time until cell death. The mathematical details of the LIB model are provided in the Appendix (Section A5).

The standard, DIP-free branch of the LIB model (figure 3.5, top row) has been used and extensively validated for influenza A virus infections [37, 38, 81, 85]. In order to explicitly capture influenza A virus infection kinetics in the presence of DIPs, the model was expanded (figure 3.5, bottom row) to incorporate two important aspects of DIP co-infection: the co-infection window, and the fraction of progeny STV produced by a co-infected cell. The co-infection window is defined as the duration post-infection for which a newly influenza A STV-infected cell remains susceptible to co-infection by DIPs. 
The LIB model allows for the co-infection window to vary in length from $0 \mathrm{~h}$ up to, and not exceeding, the length of the eclipse phase. In principle, the co-infection window could extend beyond the end of the eclipse phase, i.e., beyond the time at which a cell has begun producing and releasing STV. This is not the case for infection with influenza A STV, as explained in the Discussion.

The co-infection window introduces a second route to co-infection, i.e., STV-first, followed by DIP. It is important to note that the co-infection window in the LIB model is only defined for co-infection by STV-then-DIP, and not DIP-then-STV. In the case of the latter, the LIB model assumes that DIP-only infected cell will remain in the arrested state forever (infinitely long co-infection window), until they are co-infected by STV. This asymmetry arises because the LIB model is constructed with influenza A DIPs in mind, and assumes DIPs contain a large deletion in one of the vRNA segments that encode viral polymerase. If the LIB model considered DIPs containing DI vRNAs of other gene segments, the timing of co-infection with DIP-then-STV could require its own co-infection window, like that for STV-then-DIP. For example, if the defect in DIPs was such that their replication could proceed to shutdown of vRNA synthesis, delayed co-infection by DIP-then-STV could abolish production of both progeny, as was explored via a mathematical model in [60].

The other important aspect of DIP co-infection is the fraction of progeny STV produced by co-infected cells. In the LIB model, this is controlled by a fraction $\varepsilon$ which can take a value between 0 (co-infected cells produce only DIPs) and 1 (co-infected cells produce only STV). The LIB model assumes $\varepsilon$ is independent of the number of infecting STV and DIP. However, such a dependence might be warranted if the number of infecting particles could reverse DIP-mediated interference for influenza A virus, as suggested by [1]. In Appendix Section A2, we explain why the experimental results in [1] do not provide definitive evidence for the reversal of DIP interference, hence this process is neglected in the LIB model. Throughout our work below, co-infected cells are assumed to exclusively produce DIPs $(\varepsilon=0)$, unless otherwise stated.

Finally, because the duration of incubation prior to rinsing of the inoculum, the rate of infection, virus diffusion, affinity for cells, etc., are all factors which affect the measured infectivity of a STV or DIP in a sample, infectivity is an inherently relative quantitative measure. For the purpose of the present work, when we refer to a sample as containing $0.01 \mathrm{PFU} /$ cell, we mean one which results in the infection of $1 \%$ of cells in the context of that experiment, that is given the virus infection rate or affinity, its rate of loss of infectivity, and the incubation time if the inoculum is rinsed. Similarly, we describe a sample as containing $0.01 \mathrm{DIP} /$ cell if it results in $1 \%$ of cells becoming 
DIP-only or DIP+STV infected in the context of that same experiment.

\subsubsection{Effect of the co-infection window}

In figure 3.6, the LIB model was used to simulate the B\&C assay by infecting cells with an inoculum of DIP-free STV stock at $4 \mathrm{PFU} /$ cell plus varying dilutions of an example sample at $8 \mathrm{DIP} /$ cell when undiluted. The co-infection window length is varied from $0 \mathrm{~h}$ (i.e., a cell infected by STV first cannot be co-infected by DIP) to the length of the eclipse phase, $6.6 \mathrm{~h}$ in this example (i.e., a cell infected by STV first remains susceptible to co-infection by DIP right up until it starts to produce and release STV). As the length of the co-infection window is increased, the slope of the simulated data increases, causing the estimate for the number of DIP in the example sample based on the B\&C calculation to increase. Therefore, the length of the co-infection window relative to the eclipse length, has an important impact on the DIP MOI estimated using the B\&C assay.

To better understand how the co-infection window can cause deviations from the theoretically predicted trend in the $\mathrm{B} \& \mathrm{C}$ assay, the LIB model was used to simulate an influenza A virus infection with inoculum MOIs of $4 \mathrm{PFU} /$ cell+8 DIP/cell (figure 3.6, top right). The Poisson distribution - exploited by $\mathrm{B} \& \mathrm{C}$ to relate inoculum content to the fraction of STV-only infected cells — predicts the fraction of STV-only infected cells, given the inoculum, to be $3.29 \times 10^{-4}$, with no consideration or accounting for the length of the co-infection window. At intermediate co-infection window lengths $1.5 \mathrm{~h}-4.3 \mathrm{~h}$ for a $6.6 \mathrm{~h}$ eclipse length - the B\&C assay provides an accurate count of the inoculum DIP MOI. However, when the co-infection window is either very short or very long relative to the eclipse phase, B\&C's prediction based on the Poisson distribution disagrees with the fraction of STV-only infected cells simulated with the LIB model, and yields an incorrect estimate of the inoculum DIP MOI.

The two separate processes responsible for the disagreement for very short and long co-infection windows are depicted in figure 3.6 (bottom). With the shortest co-infection window $(0 \mathrm{~h})$, infection with DIP then STV will result in co-infection, but infection with STV then DIP, is prevented. In their use of the Poisson distribution, B\&C assume that the order of infection (DIP followed by STV or STV followed by DIP) is not important. While it is easy to account for and correct this theoretically for a co-infection window of $0 \mathrm{~h}$, it becomes increasingly complicated for non-zero co-infection window lengths because the timing of the DIP infection after STV infection becomes relevant. Since shorter co-infection windows lead to fewer co-infected cells, and more STV-only infected cells producing greater STV yields, the use of the B\&C assay will result in an underestimation of the true DIP MOI. On the other hand, when the co-infection window is almost as long 


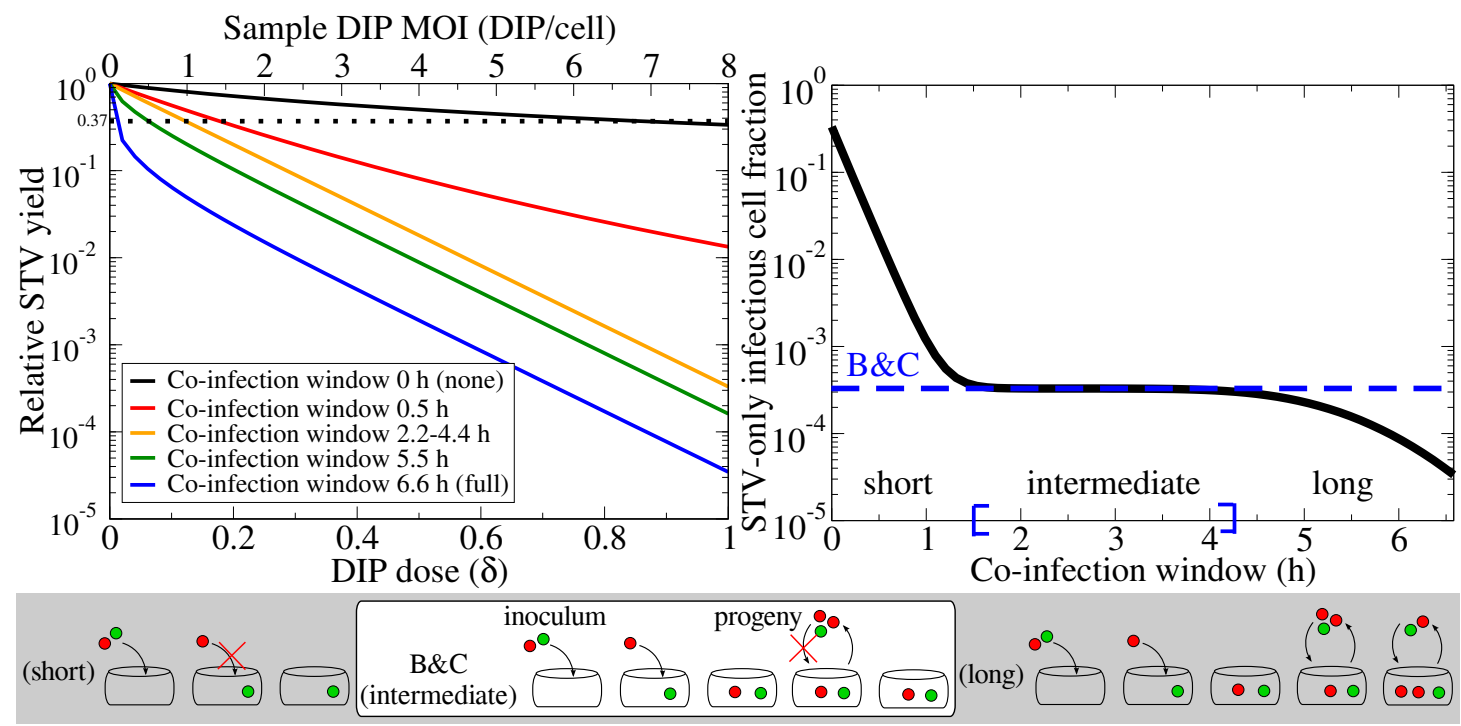

Figure 3.6: Impact of the co-infection window in the B\&C assay. (Top left) Simulated B\&C assay using the LIB model for cells infected with an inoculum of DIP-free influenza A STV stock at $4 \mathrm{PFU} /$ cell and varying dilutions of an example sample containing $8 \mathrm{DIP} /$ cell when undiluted. The various lines correspond to various co-infection window lengths. (Top right) The B\&C estimate for the fraction of STV-only infectious cells (dashed line) compared against the actual number predicted by the LIB model (solid line) for various co-infection window lengths (inoculum $=4 \mathrm{PFU} /$ cell $+8 \mathrm{DIP} /$ cell). (Bottom) For a short co-infection window, cells which receive at least one PFU will immediately exclude co-infection by DIPs. For a long co-infection window, cells remain susceptible to co-infection long enough to be co-infected by DIP progeny rather than only by the initial inoculum. For an intermediate window, the Poisson distribution on which $\mathrm{B} \& \mathrm{C}$ rely correctly accounts for the relationship between the initial infecting inoculum and STV yield. The B\&C estimate is valid for co-infection windows from $1.5 \mathrm{~h}$ to $4.3 \mathrm{~h}$ given an eclipse phase of $6.6 \mathrm{~h}$. 
as the eclipse phase, the first cells to be infected by the inoculum will begin producing progeny while the last cells to be infected remain susceptible to co-infection such that a number of these cells will be co-infected by the DIP progeny of cells infected early. B\&C account only for infection by the initial inoculum, and not for secondary infection from the progeny. Long co-infection windows will result in more co-infected cells, fewer STVonly infected cells, and therefore less STV yields than predicted by B\&C. As such, use of $\mathrm{B} \& \mathrm{C}$ with viruses that have a long co-infection window will result in an overestimation of the true DIP MOI of the sample.

\subsubsection{Length of an influenza A virus co-infection window}

Given this restriction on the validity of the $\mathrm{B} \& \mathrm{C}$ method, i.e., that the co-infection window be of intermediate length relative to the eclipse phase, it is necessary to determine a STV's co-infection window length prior to using the B\&C assay to ensure the latter will yield a correct estimate. In 1978, Nayak et al. performed an experiment with influenza A STV and DIPs which provides a good estimate of the co-infection window length [75]. Cell cultures were inoculated with a DIP-free, influenza A STV stock (1 PFU/cell, adsorbed for $30 \mathrm{~min}$ ), and at various times prior to, concurrent with, or after, were inoculated again with a sample containing a high MOI of DIPs (approximately $0.004 \mathrm{PFU} /$ cell+4 DIP/cell, adsorbed for $30 \mathrm{~min}$ ). At $14 \mathrm{~h}$ after inoculation with the DIP-free STV stock, the supernatant was harvested for subsequent quantification of the STV yield.

In order to identify the co-infection window for influenza A STV from the data generated by Nayak et al., the LIB model was fitted to the dataset, as shown in figure 3.7. The fitting procedure identified a $3.5 \mathrm{~h}$ co-infection window which is an intermediate length relative to the eclipse length, estimated as $7.1 \mathrm{~h}$ based on these data. Hence, the co-infection window of this influenza A STV strain makes it suitable for use of the B\&C assay to obtain accurate estimates of the DIP MOI. This result is discussed further in Appendix Section A1. To obtain a more accurate estimate for the co-infection window, this experiment should be performed using a higher MOI of DIP-free STV inoculum at 4 PFU/cell (see Appendix Section A5).

\subsubsection{Effect of allowing co-infected cells to produce progeny STV}

The calculation behind the B\&C assay relies on the assumption that cells co-infected with $\mathrm{DIP}+\mathrm{STV}$ will produce only DIPs, and no STV progeny. In figure 3.8, the B\&C STV yield reduction assay is simulated using the LIB model as the fraction of STV progeny 


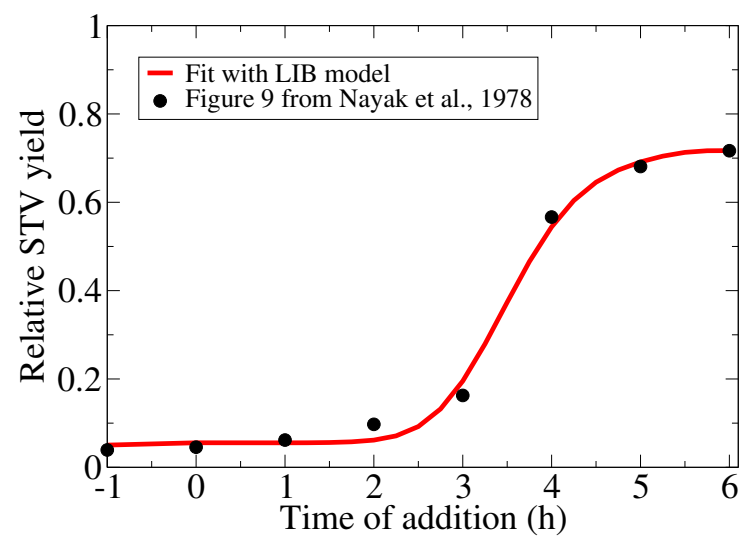

Figure 3.7: The length of the co-infection window for influenza A virus. Cells were infected with a DIP-free, influenza A STV stock at $\sim 1 \mathrm{PFU} /$ cell, followed by the addition of a DIP-containing sample at $\sim 2$ DIP/cell at various times post-infection $(x-$ axis). The STV yield is sampled $14 \mathrm{~h}$ after infection with the STV stock, and expressed relative to the STV yield from a STV stock-only (DIP-free) infection ( $y$-axis). The best fit of the LIB model to the data taken from figure 9 in Nayak et al. [75] identifies a $3.5 \mathrm{~h}$ co-infection window, relative to a $7.1 \mathrm{~h}$ eclipse length (for fitting details, see Appendix Section A5).

produced by co-infected cells, $\varepsilon$, is varied from $10^{-5}$ to $0.1 \mathrm{STV}$ (PFU) produced per DIP, or 10 to 100,000 DIPs produced per STV (PFU).

With more STV progeny produced per DIP by co-infected cells (larger $\varepsilon$ ), the B\&C curve bends upwards and a saturation in the STV yield reduction is observed. Saturation is attained when the DIP dose is sufficient to co-infect all cells, and the saturation value is equal to the fraction of STV produced per DIP by co-infected cells, $\varepsilon$. With fewer STV progeny produced by co-infected cells (smaller $\varepsilon$ ), the B\&C curve is more consistent with its theoretically predicted/assumed linear trend (i.e., no saturation). Since experimental variability in measurements of STV yield is typically greater than half an order of magnitude, the production of less than $10^{-3}$ PFU/DIP by co-infected cells would likely yield a B\&C curve that is statistically indistinguishable from, i.e., looks consistent with, the theoretically predicted trend which assumes no STV yield by coinfected cells. Thus, use of the B\&C assay — and its assumption that co-infected cells produce no STV progeny - should provide a reasonable estimate of the DIP MOI of a sample as long as co-infected cells produce no more than 1 PFU per 1,000 progeny DIPs produced. 


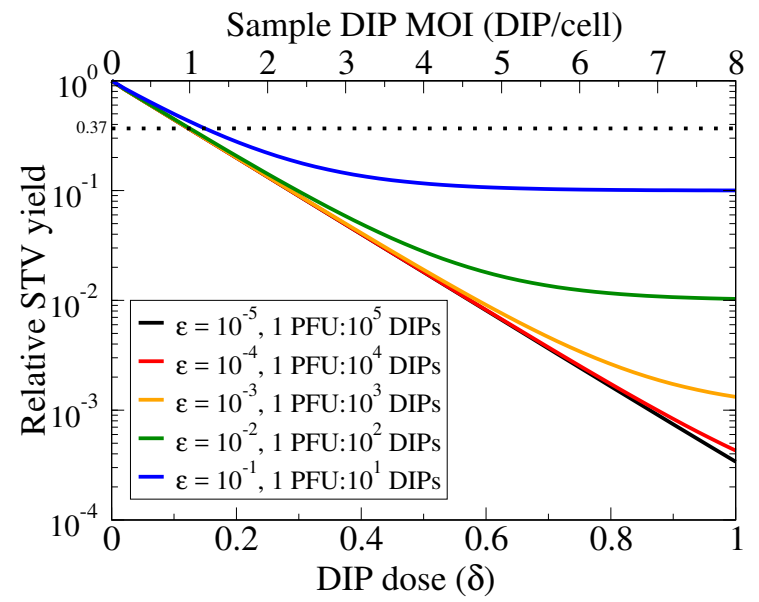

Figure 3.8: Impact of non-negligible STV yield by co-infected cells in the $\mathbf{B} \& \mathbf{C}$ assay. Simulated B\&C assay using the LIB model for cells infected with an inoculum of DIP-free influenza A STV stock at $4 \mathrm{PFU} /$ cell and varying dilutions of an example sample containing $8 \mathrm{DIP} /$ cell when undiluted. The various lines correspond to various fractions, $\varepsilon$, of STV per DIP produced by co-infected cells. For STV production by co-infected cells below 1 PFU per 1,000 DIPs $\left(\varepsilon=10^{-3}\right)$, the deviation from the theoretically predicted trend for the $\mathrm{B} \& \mathrm{C}$ curve is relatively small, i.e., it likely would not be statistically significant given the typical experimental uncertainty in STV yield measurements, and should yield reasonably accurate estimates for the DIP MOI of a sample. 


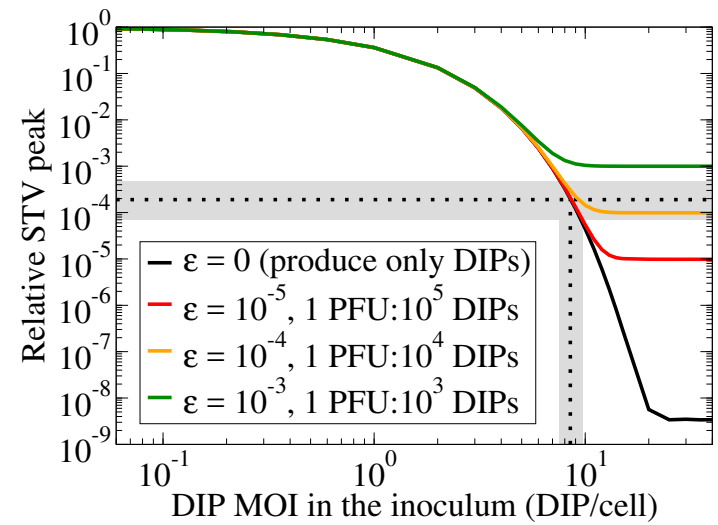

Figure 3.9: STV produced by cells co-infected with influenza A STV and DIPs. The simulated relative STV peak ( $y$-axis) as a function of the possible, unknown DIP MOI in the 2009 influenza A H1N1 pandemic virus sample ( $x$-axis). The various lines correspond to various fractions of STV produced by DIP +STV co-infected cells. The experimentally observed (figure 3.2) relative STV peak of $2 \times 10^{-4}$ is indicated (horizontal dashed line) along with its $95 \%$ confidence interval (horizontal grey bar). Cells co-infected with this 2009 influenza A pandemic STV+DIP would have to produce no more than $1 \mathrm{PFU}$ per 10,000 DIPs $\left(\varepsilon \leq 10^{-4}\right)$ in order to reproduce the relative STV peak observed experimentally, and the MOI of DIPs in the inoculum was likely $\sim 8.5 \mathrm{DIP} /$ cell (vertical dash line indicating where the curves cross the horizontal dashed line).

\subsubsection{STV production by cells co-infected by influenza A STV and DIP}

In figure 3.2, the presence of DIPs in a high MOI infection with a 2009 influenza A H1N1 pandemic virus strain was shown to decrease peak STV titer by 5,000-fold relative to an infection inoculated at low MOI. To reproduce the effect seen in this experiment, two infections were simulated with the LIB model: one receiving $4 \mathrm{PFU} /$ cell and no DIPs, the other receiving $4 \mathrm{PFU} /$ cell and an unknown DIP MOI. The STV peak from the DIP-containing infection relative to that of the DIP-free infection, was computed and compared to the $2 \times 10^{-4}(1 / 5,000)$ relative peak drop observed with the 2009 influenza A H1N1 pandemic virus strain sample. In figure 3.9, this relative STV peak is shown as a function of the possible DIP MOI in the sample, under various assumptions regarding the fraction of STV progeny produced by co-infected cells.

When co-infected cells are assumed to exclusively produce DIPs, an initial inoculum containing 8.5 DIP/cell (7.5 DIP/cell-9.7 DIP/cell) is required to match the experimentally observed relative STV peak. Given that the inoculum contained $4 \mathrm{PFU} / \mathrm{cell}$, the experimental sample of 2009 influenza A pandemic virus used to inoculate the infections 
shown in figure 3.2 likely contained 1.88 DIP/PFU-2.43 DIP/PFU, or about twice as many DIPs than PFUs.

If co-infected cells do indeed produce some STV progeny, they would have to produce less than $1 \mathrm{PFU}$ for every $10^{4}$ progeny DIPs $\left(\varepsilon \leq 10^{-4}\right)$ in order to reproduce the observed relative STV peak. Hence, cells co-infected with this influenza A STV+DIP could not produce more than 1 PFU for every $10^{4}$ progeny DIPs, a level of STV production that is negligible in the context of the $\mathrm{B} \& \mathrm{C}$ assay, as shown in figure 3.8. Thus, use of the $\mathrm{B} \& \mathrm{C}$ assay should be appropriate to estimate the DIP MOI for this influenza A virus strain (A/Québec/144147/09 (H1N1)) because it produces less than the 1 PFU per 1,000 progeny DIPs required for the $\mathrm{B} \& \mathrm{C}$ assay to remain accurate. This result is discussed further in Appendix Section A1.

\subsubsection{Effect of using STV stock at low MOI to perform the B\&C assay}

Herein, thus far, the following requirements have been identified to ensure the $\mathrm{B} \& \mathrm{C}$ assay is suitable to accurately quantify DIPs: (1) A co-infection window of intermediate length relative to the eclipse phase; and (2) Cells co-infected with DIP+STV must produce fewer than 1 PFU for every 1,000 progeny DIPs. Our analysis of experiments performed with various influenza A viruses suggest that this virus does appear to meet these requirements. Yet, in figure 3.4, important deviations from the theoretically predicted linear trend can be observed for the B\&C assay performed by Marcus et al. to quantify influenza A DIPs [65]. Thus, there must be additional criteria to be met in order for the B\&C assay to function properly. In [65], Marcus et al. cite the findings of Akkina et al. [1] — namely, the reversal of DIP-mediated interference at high STV MOIs (see Appendix Section A2) - to justify conducting their B\&C assay with a low MOI of $0.3 \mathrm{PFU} /$ cell, rather than a high MOI, of their relatively DIP-free STV stock (obtained by passaging at low MOI). Unfortunately, such a low STV MOI leaves $74 \%$ $\left(\mathrm{e}^{-0.3} \times 100 \%\right)$ of cells uninfected by STV, and thus remaining susceptible to infection by the DIP and STV progeny from a second cycle of infection. As discussed above, the $\mathrm{B} \& \mathrm{C}$ calculation relies exclusively on the content of the inoculum and does not account for infections resulting from a second cycle of infection.

In figure 3.10 (left), the B\&C assay is simulated for infections inoculated with varying MOIs of the DIP-free STV stock plus a series of dilutions of an example sample which contains $8 \mathrm{DIP} /$ cell when undiluted. The simulations assume a $3 \mathrm{~h}$ co-infection window, relative to the $6.6 \mathrm{~h}$ eclipse length, and assume that co-infected cells exclusively produce DIPs, i.e., produce no STV $(\varepsilon=0)$. As the STV MOI in the DIP-free STV stock is decreased, the simulated $\mathrm{B} \& \mathrm{C}$ assay deviates from the theoretically predicted linear trend 
at low DIP doses, i.e., at high dilutions of the DIP-containing sample being measured. The desired linear trend, yielding a correct DIP count, is obtained when using a DIP-free STV stock of $4 \mathrm{PFU} /$ cell, but not for STV MOIs at, or below, $1 \mathrm{PFU} /$ cell. When using a DIP-free STV stock at MOIs that are too low, the B\&C assay always overestimates the DIP MOI in the sample. As shown in figure 3.10 (right), at a STV stock MOI of 2.5 PFU/cell used by Bellett and Cooper [6], the B\&C assay overestimates the DIP MOI by $\sim 10 \%$, or 1.1-fold, an error that is likely smaller than the experimental uncertainty expected in an actual experiment. At the STV MOI of $0.3 \mathrm{PFU} /$ cell used by Marcus et al. [65], the overestimate would be no less than $1600 \%$, or at least 17 times larger than the true DIP MOI in the sample. Generally, when using the B\&C assay to estimate the MOI of DIPs in a sample containing a high MOI of DIPs (at least more than $0.1 \mathrm{DIP} /$ cell undiluted), a DIP-free STV stock MOI of no less than $3 \mathrm{PFU} /$ cell should be used to keep the error in the estimate below $5 \%$. Otherwise, if the sample contains a low MOI of DIPs (less than 0.1 DIP/cell undiluted), then an even higher DIP-free STV stock MOI is needed to keep the error in the estimate around 5\%, as shown in figure 3.10 (right).

The causes of curvature are summarized in Appendix Section A4, which includes an explanation of the B\&C's assay sensitivity to the DIP MOI of the sample (Appendix Section A3). 

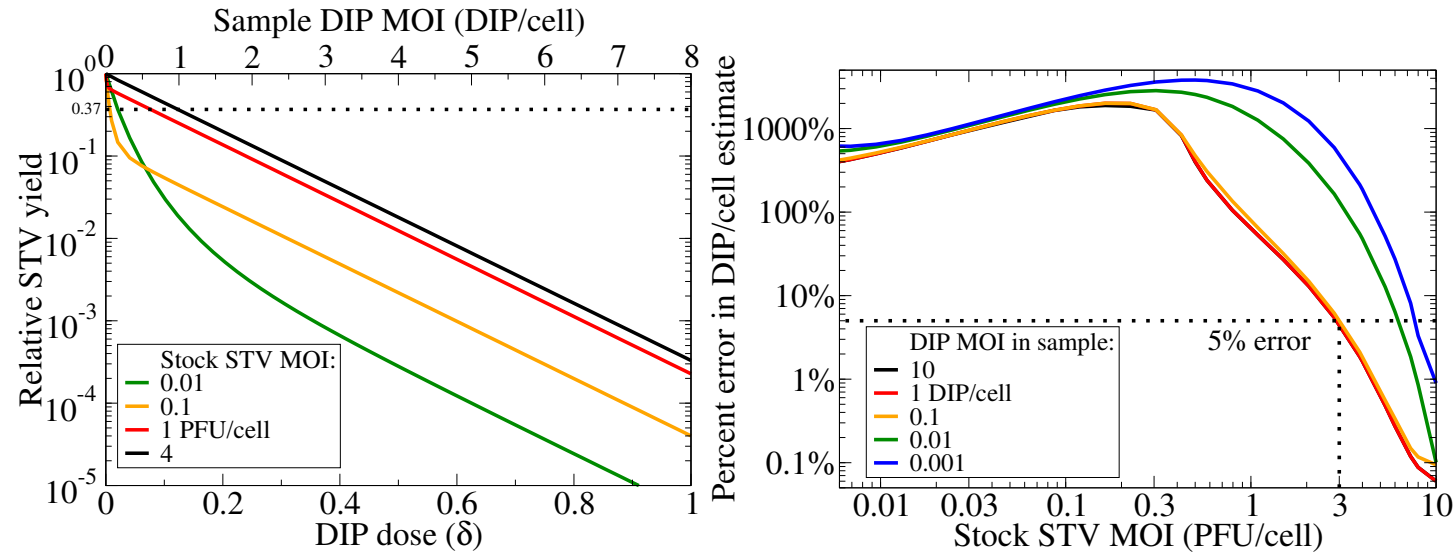

Figure 3.10: Impact of the STV MOI of the DIP-free STV stock. (Left) Simulated $\mathrm{B} \& \mathrm{C}$ assay for inoculation with serial dilutions of an example sample containing $8 \mathrm{DIP} /$ cell when undiluted, plus a DIP-free STV stock. The various lines correspond to various MOIs of the DIP-free STV stock used in conducting the assay. (Right) The relative percent-error in the DIP MOI estimated using the B\&C assay - when computed from the reciprocal of the sample dilution at $37 \%$ relative STV yield - as a function of the DIP-free STV stock MOI used. The various lines correspond to the actual DIP MOIs in the undiluted sample being evaluated through the $\mathrm{B} \& \mathrm{C}$ assay. The $\mathrm{B} \& \mathrm{C}$ assay is most accurate (error $<5 \%)$ for high DIP sample $(>0.1 \mathrm{DIP} /$ cell) and STV stock (>3 PFU/cell) MOIs. 


\subsection{Discussion}

In 1959, Bellett \& Cooper (B\&C) developed an in vitro assay to quantify defective interfering particles (DIPs). The calculations underlying B\&C's method dictate that the assay curve be a decreasing straight line, i.e., there should be a linear relationship between the logarithm of the quantity with which DIPs interfere (B\&C used the infectious standard virus (STV) yield) as a function of increasing DIP dose. From this linear relationship, the DIP MOI of an unknown sample can be estimated. In many publications making use of interference assays based on the B\&C assay, the assay data does not follow the requisite linear trend [6, 64, 65, 86, 103, 109], yet the DIP MOI was still estimated using the $\mathrm{B} \& \mathrm{C}$ calculations. The deviation of the assay data from the linear relationship has never been fully explained. We asked whether the B\&C assay could still correctly estimate the DIP MOI when these deviations are apparent, and if not, we were further interested in determining if there were any constraints on the applicability of the $\mathrm{B} \& \mathrm{C}$ assay that were not stated in the original publication [6].

We used a mathematical model of influenza A virus infection (LIB model) to simulate in vitro infections in the presence of DIPs, in order to reproduce and verify the $\mathrm{B} \& \mathrm{C}$ assay. In the LIB model, we introduced two key co-infection parameters: the co-infection window (how long after STV infection cells block further infection by other virions), and the fraction of STV to DIP progeny (PFU/DIP) produced by cells co-infected by DIP and STV. In general, our results show that the B\&C assay will correctly estimate the DIP MOI in a sample when: (1) a STV-infected cell's co-infection window is approximately half the length of its eclipse phase (how long after initial infection the cell begins to produce progeny virion); (2) cells co-infected by STV and DIP produce fewer than 1 STV per $10^{3}$ progeny DIP; and (3) the B\&C assay is performed using a high STV MOI (greater than $4 \mathrm{PFU} /$ cell) for initial infection. To our knowledge, this is the first time explicit conditions for proper use of the B\&C assay have been identified.

In order to ensure the DIP MOI of a sample can be reliably estimated by performing a $\mathrm{B} \& \mathrm{C}$ assay, one should visually verify that the data generated by their $\mathrm{B} \& \mathrm{C}$ assay follows a straight line, as described in more detail herein. If this is not the case then the curvature could be due to:

Cause 1 the use of a DIP-free STV stock which infects cells with a STV MOI that is too low. The STV stock should be concentrated until a high STV MOI can be achieved;

Cause 2 too high a DIP MOI in the sample whose DIP content is to be quantified. The sample should be diluted further before it is used to perform the B\&C assay; 
Cause 3 the key co-infection parameters of the specific virus of interest (i.e, length of co-infection window, fraction of STV produced by co-infected cells) not meeting the conditions we outline herein; and/or

Cause 4 the specific virus of interest having a mechanism of interference inconsistent with that assumed by the B\&C assay and described herein.

While the first two causes can be addressed by modifying the experimental procedure, the last two are virus-dependent and thus cannot be remediated. In such a case, a different assay would need to be designed and our findings might not apply.

Throughout our work we made several reasonable assumptions, and so, our results should be taken with the following caveats in mind. First, we assumed that the STV stock was DIP-free. Practically, we do not know how close to "DIP-free" a STV stock can be made, since the effectiveness of methods to reduce the concentration of DIPs (e.g., low MOI passaging) remain yet to be quantified. However, we expect that a STV stock containing DIPs would only diminish the B\&C assay's sensitivity to samples containing very high DIP concentrations, and this can be easily fixed by further diluting the sample whose DIP content is to be quantified using the B\&C assay. Second, we assumed that the generation of de novo DIPs by STV-only infected cells was a negligible source of DIPs, compared to the amplification of DIPs already in a sample. This is reasonable since replicase errors should occur relatively infrequently. Even if the generation of DIPs was not negligible, the LIB model predicts there would be no impact on the relative yield if the $\mathrm{B} \& \mathrm{C}$ assay is performed properly as a single-cycle infection, with an intermediate co-infection window and low fraction of STV progeny produced by co-infected cells. As such, the findings presented herein hold irrespective of these assumption. Additionally, our validation of $\mathrm{B} \& \mathrm{C}$ is linked to the assumptions made in the LIB model specific to the mechanisms of interference by influenza A DIPs. These include the assumption that DIP-only infected cells remain susceptible to STV co-infection for any length of time; that the timing of DIP+STV co-infection does not affect the amount or ratio of progeny produced; that the magnitude of the interference is independent of the number of infecting STV and DIP; and that both infected and co-infected cells produce the same amount of progeny. Herein, we provide justification for these assumptions in the context of influenza A STV and DIP infection. If a virus' mechanism for interference is different from that assumed here, then there could yet be other reasons why that virus cannot be characterized by B\&C. For example, DIPs of VSV have been shown to exhibit a multiple-hit inhibition mechanism, which is incompatible with both the LIB model and the assumptions of the B\&C assay, as shown in [54, 102]. 
To our knowledge, all assays to quantify the presence of DIPs in a sample to date rely on the same principles and calculations as the $\mathrm{B} \& \mathrm{C}$ assay, differing only in their endpoint, i.e. the $y$-axis in the $\mathrm{B} \& \mathrm{C}$ plot. $\mathrm{B} \& \mathrm{C}$ assume that $\mathrm{DIP}+\mathrm{STV}$ co-infected cells produce only DIP such that the reduction in STV yield (their endpoint) corresponds to a proportional reduction in STV-only infected cells in favour of STV+DIP co-infected cells, as performed in $[6,57,65,95,102]$. In the variation of the $\mathrm{B} \& \mathrm{C}$ assay performed in $[48$, 50, 109], after inoculation and incubation, infected and co-infected cells are trypsinized, and plated onto a new monolayer under agar. In their assay, the reduction in STV-only infected cells is counted as a reduction in visible plaques, rather than a reduction in STV yield. As such, this assay is in all ways equivalent to that performed by B\&C, and our findings apply to it as well. The other variation of the B\&C assay performed in $[64,68,86,103,114]$ assumes that cells co-infected by STV+DIP will be protected from cytopathic effects (CPE) whereas those infected by STV alone will exhibit CPE. In their assay, the reduction in STV-only infected cells is counted as a reduction in CPE, rather than a reduction in STV yield. Herein, because of our focus on influenza A virus, we have assumed both STV-only and STV+DIP infected cells will have the same lifespan and thus exhibit the same CPE profile. This assumption is supported by both our prior work [37, 38, 81, 85] and findings reported in [65], but is in contradiction with that reported in [68], all in the context of influenza A virus infections in the presence of DIPs. Nonetheless, for viruses where DIP co-infection is truly protective from CPE, use of the $\mathrm{CPE}$ as the endpoint in the $\mathrm{B} \& \mathrm{C}$ calculation will result in a valid, linear relationship only if the virus' co-infection window is of intermediate duration relative to its eclipse phase (as with STV yield reduction), but would not require that co-infected cells produce negligible amounts of STV (as is needed when using STV yield reduction). Since we did not explore such a mechanism herein, however, it is possible that using $\mathrm{CPE}$ as the endpoint requires additional constraints not explored here.

While our work explains why not obtaining a linear relationship means B\&C is not valid, we cannot guarantee that obtaining a straight line means $\mathrm{B} \& \mathrm{C}$ is valid. As such, it is important to ensure the above criteria are met even when a linear trend is obtained. The B\&C assay has already been applied to viruses such as Sindbis virus [50, 57], lymphocytic choriomeningitis virus [86, 109, 114], infectious pancreatic necrosis virus [64], respiratory syncytial virus [103], mumps virus [95], and influenza A virus [48, 65, 68]. Herein we describe reliable procedures to determine a virus' key co-infection parameters and verify Cause 3 via simple, conventional, in vitro infection experiments, using influenza A virus as an example, so that others can follow our methods with their virus of interest. We hope that our work, and that of others before us [54, 102], will motivate 
others to do so. When applied correctly to quantify the DIP content of a suitable virus, the $\mathrm{B} \& \mathrm{C}$ assay is an effective methodology to reliably monitor DIP accumulation in high yield processes, determine actual dosages when DIPs are used as antivirals, and resolve the extent to which existing methods to reduce DIP contents in virus samples are effective. For viruses that do not meet the criteria identified herein, our LIB model, with appropriate modifications to capture the specific interference mechanism of that virus, could potentially help test alternatives to the B\&C assay or even be used to directly quantify DIPs in that virus sample. 


\section{Competing interests}

We have no competing interests.

\section{Authors' contributions}

LEL and CAAB both designed the study, developed the mathematical and computer models, and performed the analysis. LEL, SI, and CAAB drafted and revised the manuscript. All authors gave final approval for publication.

\section{Funding}

This work was supported in part by a Discovery Grant (355837-2013) from the Natural Sciences and Engineering Research Council of Canada (NSERC, http://www.nserccrsng.gc.ca) and an Early Researcher Award (ER13-09-040) from the Ontario Ministry of Research and Innovation (http://www.mri.gov.on.ca), both awarded to CAAB, with additional support through the iTHES research group at RIKEN (to CAAB). The work was also supported in part by the Japan Science and Technology Agency (JST) through the PRESTO program, the JST RISTEX program, and the JST CREST program; by the Japan Society for the Promotion of Science (JSPS) KAKENHI Grant Numbers 15KT0107, 26287025, 16H04845 and 16K13777; and by the Japan Agency for Medical Research and Development, AMED, all awarded to SI. Additional funding in the form of stipends and the Michael Smith Foreign Study Supplement was provided through a Doctoral Canadian Graduate Scholarship awarded to LEL by the Natural Sciences and Engineering Research Council of Canada. 


\section{Chapter 4}

\section{Exploring virus release as a}

bottleneck for the spread of influenza A virus infection in vitro and the implications for antiviral therapy with neuraminidase inhibitors

This chapter is based on the following paper submitted for publication:

L. E. Liao, S. Kowal, D. A. Cardenas and C. A. A. Beauchemin. Exploring virus release as a bottleneck for the spread of influenza A virus infection in vitro and the implications for antiviral therapy with neuraminidase inhibitors. Submitted to PLOS ONE. 2017; PONE-D-17-22978. 


\begin{abstract}
Mathematical models (MMs) have been used to study the kinetics of influenza A virus infections under antiviral therapy, and to characterize the efficacy of antivirals such as neuraminidase inhibitors (NAIs). NAIs prevent viral neuraminidase from cleaving sialic acid receptors that bind virus progeny to the surface of infected cells, thereby inhibiting their release, suppressing infection spread. When used to study treatment with NAIs, MMs represent viral release implicitly as part of viral replication. Consequently, NAIs in such MMs do not act specifically and exclusively on virus release. We compared a MM with an explicit representation of viral release (i.e., distinct from virus production) to a simple MM without explicit release, and investigated whether parameter estimation and the estimation of NAI efficacy were affected by the use of a simple MM. Since the release rate of influenza A virus is not well-known, a broad range of release rates were considered. If the virus release rate is greater than $\sim 0.1 \mathrm{~h}^{-1}$, the simple MM provides accurate estimates of infection parameters, but underestimates NAI efficacy, which could lead to underdosing and the emergence of NAI resistance. In contrast, when release is slower than $\sim 0.1 \mathrm{~h}^{-1}$, the simple MM accurately estimates NAI efficacy, but it can significantly overestimate the infectious lifespan (i.e., the time a cell remains infectious and producing free virus), and it will significantly underestimate the total virus yield and thus the likelihood of resistance emergence. We discuss the properties of, and a possible lower bound for, the influenza A virus release rate.
\end{abstract}

\title{
4.1 Introduction
}

There are two main classes of antiviral drugs available for the treatment of influenza A virus infection: adamantanes and neuraminidase inhibitors (NAIs). In 2005-2006, however, resistance to adamantanes dramatically increased [106], and the currently circulating influenza A/H3N2 strains are adamantane-resistant. In light of this, the World Health Organization primarily recommends NAIs such as oseltamivir and zanamivir for antiviral therapy against currently circulating strains of influenza A virus [111]. As a part of pandemic preparedness planning, oseltamivir has been stockpiled worldwide. However, even oseltamivir-resistant A/H1N1 strains have emerged and circulated [107]. Recent focus has turned to the development of new antivirals that inhibit viral polymerase (e.g., favipiravir), though these have yet to be approved in most countries, leaving NAIs as the leading antiviral approved for the treatment of influenza A virus infections.

NAIs reduce the spread of influenza A virus to uninfected cells by blocking the release of progeny virus produced by infected cells. At this late step in the viral replication cycle, mature virions protrude and pinch off from the apical surface of the infected cell, co-opting the cell's plasma membrane as their own envelope, but can remain affixed 
atop the cell surface. Both the virion and the cell surface, which is destined to become the virion's outer surface, are studded with the viral proteins neuraminidase (NA) and hemagglutinin (HA), as well as the cell's sialic acid receptors. Throughout the duration of the infection, increasing amounts of NA are expressed on the cell surface, which cleave sialic acid receptors. As the density of sialic acid receptors declines, newly budded virions are less likely to remain cell-bound due to the formation of virus-cell attachments when HA binds to the sialic acid receptors upon exit. We will refer to the transition from cell-associated, bound virus into free virus that is facilitated by NA cleavage of sialic cell receptors as "virus release", though other modes of virus release might exist[30, 44, 63].

As reviewed in [3], a simple MM has provided insight into influenza A virus infection kinetics in both in vitro and in vivo settings. The simple MM has been used to study NAI therapy in humans that were infected with human strains [2] or avian strains of influenza A virus [17], and the MM has been extended to include an immune response [18, 32]. Although the simple MM has been used to study the inhibition of virus release by NAIs, it does not possess an explicit representation of virus release. In the simple MM, virus release is implicitly represented as part of virus replication which encompasses many processes, shown in Figure 4.1, such as viral transcription and translation, up to later events such as bud initiation, bud growth and closure, and finally virus release. Consequently, when NAIs are incorporated into the simple MM, they act on these combined processes instead of acting specifically and exclusively on virus release.

If virus release plays a significant role in the unfolding infection kinetics, the use of a MM without explicit virus release, like the simple MM, could affect the estimation of infection parameters, with broad implications. For instance, an estimate of the virus production rate can shape our perspective on the likelihood of the emergence of drug resistance $[36,82,89]$. In a scenario where rapid virus production is undermined by slow virus release from an infected cell, only the overall slow rate of appearance of progeny virus in the supernatant would be observed when the infectious virus yield is collected in vitro. If the simple MM, where the virus release rate is implicitly represented in the virus production rate, was used to fit these data, the estimated virus production rate would be an underestimation of the true rate. Since the chance of generating drug-resistant mutants with a single amino acid substitution is predicted to increase as more virus genomes are produced [83], underestimation of the virus production rate will underestimate the likelihood of drug resistance emergence and the number of drugs, or efficacy, required to prevent resistance $[40,91]$.

The importance of quantifying the infection parameters from time course data of an in vitro infection with a MM has been discussed [46]. This approach allows for the 


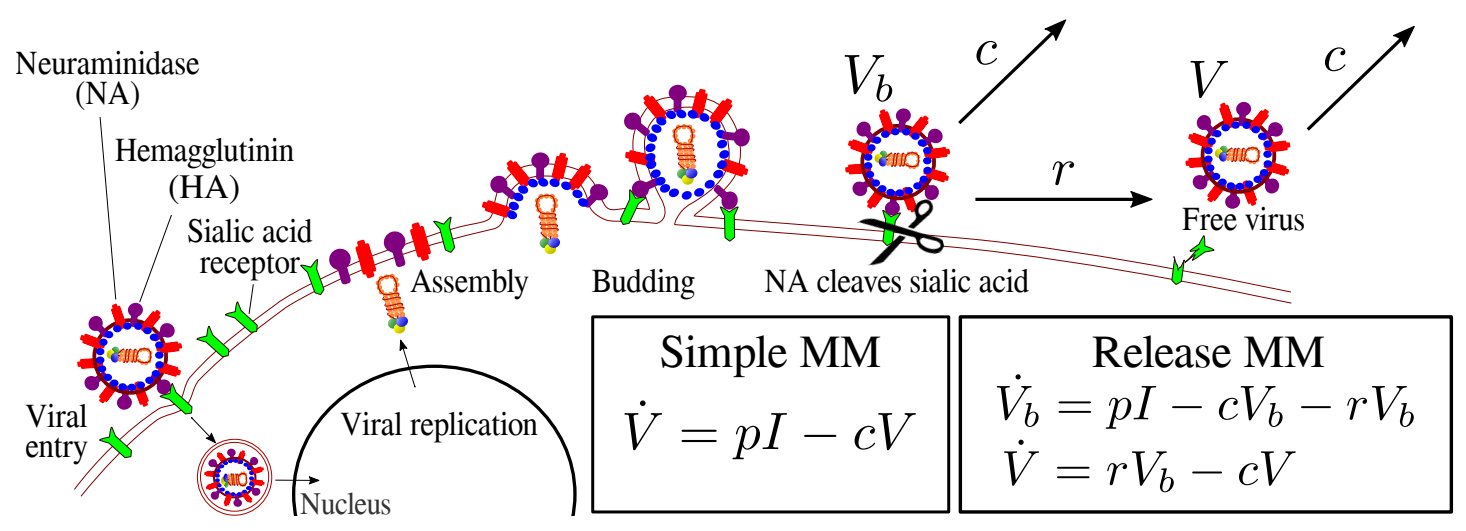

Figure 4.1: Modelling influenza A virus infection with and without explicit release. In an influenza $A$ virus infection, the virion gains entry into the cell when hemagglutinin (HA) proteins on the surface of virions bind to sialic acid receptors on the surface of the target cell. As viral replication gets underway, increasing amounts of viral proteins such as HA and neuraminidase (NA) are expressed on the cell surface. Throughout the infection, the density of sialic acid receptors declines as NA cleaves them. After viral replication takes place in the nucleus, the viral RNA progeny is transported to the cell membrane for virus assembly and budding. Some progeny virions will be released as free virus $(V)$, while others remain bound $\left(V_{b}\right)$ to the cell surface upon exiting the cell when HA on the surface of budded virion binds to sialic acid on the cell. The simple MM without an explicit term for viral release encapsulates these later processes implicitly as a part of the parameter quantifying free virus production $(p)$ by infectious cells $(I)$. The release MM has an explicit term for viral release at a rate $r$, which occurs after bound virus is produced at a rate $p$ onto the surface of the cell. In both MMs, all virions, bound $\left(V_{b}\right)$ or free $(V)$, lose infectivity at rate $c$. 
quantitative comparison of differences between virus strains, for example, in the virus production rate, cell lifespan, or basic reproductive number, which are indicators of virulence, cytopathicity, and fitness. These quantities, even if determined only from in vitro infections, can inform and to an extent predict epidemiological outcomes such as pathogenicity and transmissibility [71].

The challenge to including virus release explicitly in a MM is that the influenza $\mathrm{A}$ virus release rate is not well-known. From a mathematical modelling standpoint, the addition of an explicit release rate to the simple MM will result in overparameterization, such that at least some parameters would suffer with identifiability issues. To overcome this challenge, our study considers a range of possible values of the release rate of influenza A virus, and compares the simple MM against a variation of it that includes an explicit term for virus release (Figure 4.1). We identify the critical release rate below which virus release begins to play a significant role in determining the levels of free (i.e., no longer bound to the cell) infectious virus. We show how neglecting to account for virus release with an explicit release term affects parameter estimation, as well as estimates of NAI efficacy. 


\subsection{Results}

\subsubsection{Considering an explicit release rate and the impact on viral ki- netics}

We compared a simple MM (without explicit virus release) to a MM with explicit virus release (hereafter, referred to as the release MM). As described in the Methods (Section 4.4), the release MM is an augmented version of the simple MM with one additional parameter, the virus release rate $r$. Due to this small modification, the MMs have different views of virus infection, and some parameters in the two MMs require a slightly different interpretation. For example, in the simple MM, the eclipse length, $\tau_{E}$, represents the time it takes for an infected cell to replicate virus and release it. In contrast, in the release MM, the eclipse length only represents the time it takes for the infected cell to replicate virus, but the time to viral release is handled separately with parameter $r$. The interpretation of the virus production rate, $p$, in either MM also differs: whereas in the release MM, a fraction of the virions produced will never be released if the release rate is low, in the simple MM, all produced virions are released. Thus, in the simple MM, the effects of virus release can be absorbed by either or both the virus production rate and eclipse length.

Another difference from the simple MM, is that the release MM explicitly describes cell-associated, bound infectious virus $\left(V_{b}\right)$ being released at rate $r$ as free infectious virus $(V)$ into the medium. When we refer to bound virus, we mean infectious virions that are still attached to the virion-producing cell, i.e., cell-associated infectious virus. Experimentally, bound virus is not measurable in a typical cell culture experiment where only the supernatant is removed and titrated for infectious virus. On the other hand, free virus here refers to infectious virus that has successfully been released from virusproducing cells into the supernatant, and corresponds to the population of virus that is typically quantified in an experiment.

We investigated the impact of the release $\mathrm{MM}$ on the kinetics of free and bound virus by comparing it to that predicted by the simple MM. In Figure 4.2A, a baseline simulation of the free virus kinetics predicted by the simple MM is shown for the case of a virus infection in vitro where the infectious virus released into the supernatant was quantified by plaque forming assay [85]. The release MM was used to simulate the same virus infection, with the same parameter values used with the simple MM, but the release rate was varied since the value of the influenza A virus release rate is unknown.

Compared to the simple MM simulation, the growth of free virus in the release MM was only suppressed when the virus release rate fell below some critical value. We call 

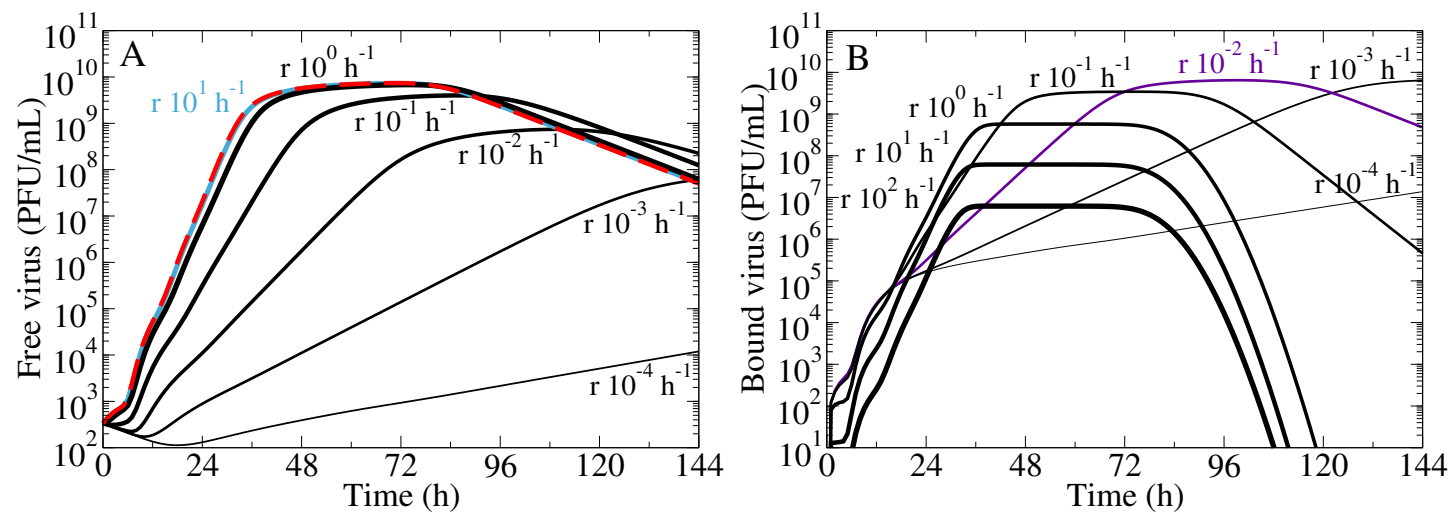

Figure 4.2: The role of explicit viral release on free and bound virus kinetics. (A) The concentration of free virus titer from an in vitro infection experiment with the 2009 pandemic influenza A (H1N1) virus strain [85] was simulated in the simple MM (red), which serves as our baseline simulation. The baseline is compared to the free virus titer simulated in the release MM (black) where the release rate, $r$, is varied from $10^{-4} \mathrm{~h}^{-1}$ to $10^{2} \mathrm{~h}^{-1}$, and all other parameters are kept at their base values. Note that when $r \geq 100 \mathrm{~h}^{-1}$, the release MM reduces to the simple MM. For low release rates, the free virus is suppressed compared to the baseline (black vs red), but is unaffected when $r$ is greater than a critical value of $r_{f}=3 \mathrm{~h}^{-1}-5 \mathrm{~h}^{-1}$ (teal). (B) The corresponding concentration of bound virus in the release MM is shown (black), where the bound virus titer peak is maximal at another critical value of the release rate, $r_{b}=3 \times 10^{-3} \mathrm{~h}^{-1}$ (purple).

this value the critical free virus release rate, $r_{f}$, and determined that it ranges between $3 \mathrm{~h}^{-1}-5 \mathrm{~h}^{-1}$ (see Appendix Section B.1 for details of this estimation). If the virus release rate is below this critical value, virus release is slow and inefficient and plays a significant role in suppressing free virus yield.

The release MM also predicts the effect of virus release on the kinetics of bound virus. In Figure 4.2B, the corresponding bound virus titer predicted for various release rates is shown. Note that this quantity is zero in the simple MM which assumes that all produced virus is released. Increasing the release rate from $10^{-4} \mathrm{~h}^{-1}$ to $10^{-2} \mathrm{~h}^{-1}$ increased the bound virus peak, but a release rate greater than $10^{-2} \mathrm{~h}^{-1}$ diminished the bound virus peak. There appears to be a value of the release rate that maximizes the bound virus titer peak, which we call the critical bound virus release rate, $r_{b}$. We determined that $r_{b}$ was approximately $3 \times 10^{-3} \mathrm{~h}^{-1}$, though its value depends on other infection parameters (see Appendix Section B.1). When the release rate is high, virions are released as soon as they are produced, and the amount of bound virus is negligible. As the release rate is lowered, towards $r_{b}$, produced virus remains attached longer and the concentration of 
bound virus increases. However, as the release rate is lowered even further, below $r_{b}$, virions are trapped on the cell surface for so long that some will lose infectivity (their ability to infect other cells) before they are released, resulting in a decrease in the bound virus titer. A balance between these two processes results in the largest bound virus titer peak value.

Our results show that virus release can act as a kinetic bottleneck to significantly suppress free virus growth if the true rate of influenza A virus release is less than the critical free virus release rate, $r_{f}$. In such a case where virus release plays a significant role in virus infections, release must be explicitly accounted for in the MM.

\subsubsection{The effect of explicit virus release on parameter estimates}

Most MMs to date have represented the virus release as an implicit part of virus replication. Here, we are interested in how parameter estimation using the simple MM compares to that using the release MM over a wide range of possible release rates.

We have previously shown that a full suite of in vitro experiments - single-cycle (SC), multiple-cycle (MC), mock-yield (MY) - is needed to extract and identify the infection parameters (e.g., virus production rate, infection rate, rate of loss of virion infectivity, eclipse length, infectious lifespan) in the simple MM [81, 85, 98]. Briefly, the MC and $\mathrm{SC}$ assays are in vitro infections where a monolayer of confluent, uninfected cells are inoculated with a low or high concentration of virus, respectively. The initial conditions of infection are expressed in terms of multiplicity of infection (MOI), i.e., the number of cells infected by the initial inoculum over the total number of cells. Herein, the simulated MC assay was carried out at a MOI of $5 \times 10^{-5}$ infected cells/cell, while the SC assay was performed at a MOI of 4 infected cells/cell. In the MY assay, the virus inoculum is incubated in the absence of cells and sampled at multiple times, exhibiting the loss of infectious virions due to thermal inactivation. Figure 4.3 shows the free virus kinetics from these assays, as simulated by the simple MM using the base parameters reported in Table 4.1, and by the release MM.

For the release MM, the release rate was fixed, and the remaining five MM parameters were fitted using the simulated free virus titer from the simple MM as the data to fit (red). A set of fitted parameters was obtained for each value of the release rate explored. The release MM can reproduce the simple MM's infection kinetics with a unique set of infection parameters, for any given value of the release rate. This shows how the parameter estimates shift under different assumptions of the release rate.

Figure 4.4 shows the infection parameters predicted by the release MM as a function of the release rate, $r$. If free virus release is rapid and effective $\left(r>r_{f}\right)$, the simple MM 

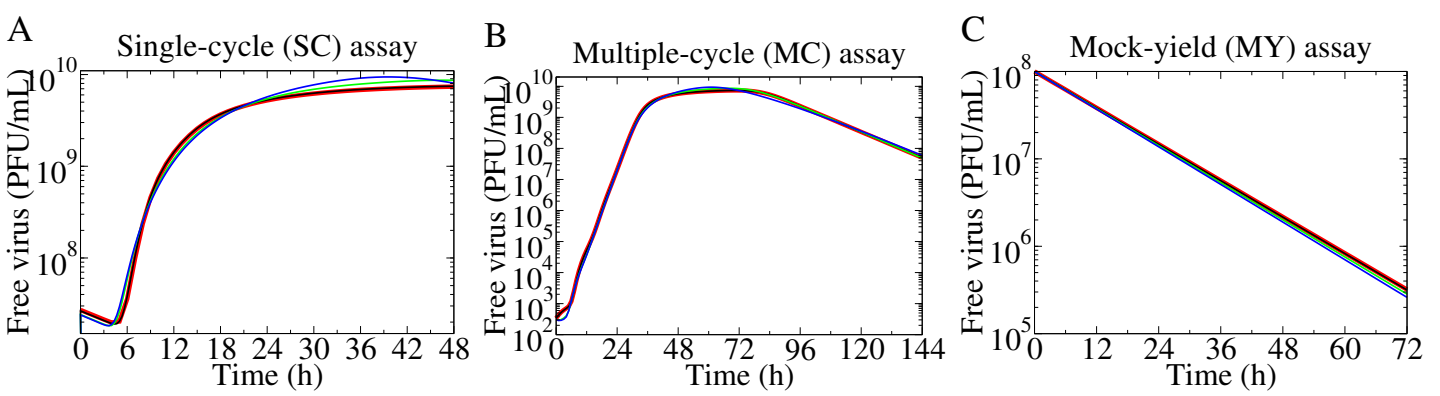

Figure 4.3: Simultaneous fits of the release MM to the simulated free virus titer of the SC, MC, MY assays. The release MM was simultaneously fitted to the free virus titer from the (A) SC, (B) MC, and (C) MY assays simulated using the simple MM (red; using base parameters). The production rate, infection rate, rate of loss of virion infectivity, eclipse length, and infectious lifespan were fitted. Fitted curves are shown for a high $\left(r=1 \mathrm{~h}^{-1}\right.$, black $)$, an intermediate $\left(r=0.1 \mathrm{~h}^{-1}\right.$, green), and a low $\left(r=0.01 \mathrm{~h}^{-1}\right.$, blue) release rate.

returns the same parameter estimates as predicted by the release MM, and use of the simple MM is appropriate in this circumstance. If free virus release is slow and inefficient $\left(r<r_{f}\right)$, the simple MM no longer correctly estimates the infection parameters.

In particular, Figure 4.4 shows that the simple MM significantly overestimates the eclipse length, i.e., the time a cell remains infected but not yet producing free virus (Figure 4.4A), and infectious cell lifespan, i.e., the time a cell remains infectious and producing free virus (Figure 4.4B), and underestimates the virus production rate (Figure $4.4 \mathrm{C})$. The remaining fitted parameters do not significantly differ between the MMs (Appendix Figure B.4).

As previously described, the interpretation of the eclipse length differs between the two MMs and this is reflected in the estimate of $\tau_{E}$ obtained from the fits. In Figure 4.3A, the SC assay shows an approximately $6.7 \mathrm{~h}$ delay before the growth of free virus is measured. If virus release is assumed to be rapid $\left(r>r_{f}\right)$, the release MM ascribes the full delay to the eclipse length which represents the viral replication time. On the other hand, if virus release is assumed to be slow $\left(r<r_{f}\right)$, the release MM ascribes only part of the delay to the eclipse length (time for viral replication), while the rest is ascribed to the time required to release virus. Consequently, as the release rate decreases, less and less of the delay is ascribed to virus replication (the estimated eclipse length decreases) while the time required to release virions increases more and more. This highlights the release MM's ability to ascribe the delay discriminately to each process, i.e., separately to virus replication and release. In the simple MM, the full delay is ascribed to the eclipse length, since parameter $\tau_{E}$ accounts for both the viral replication time and release time. 

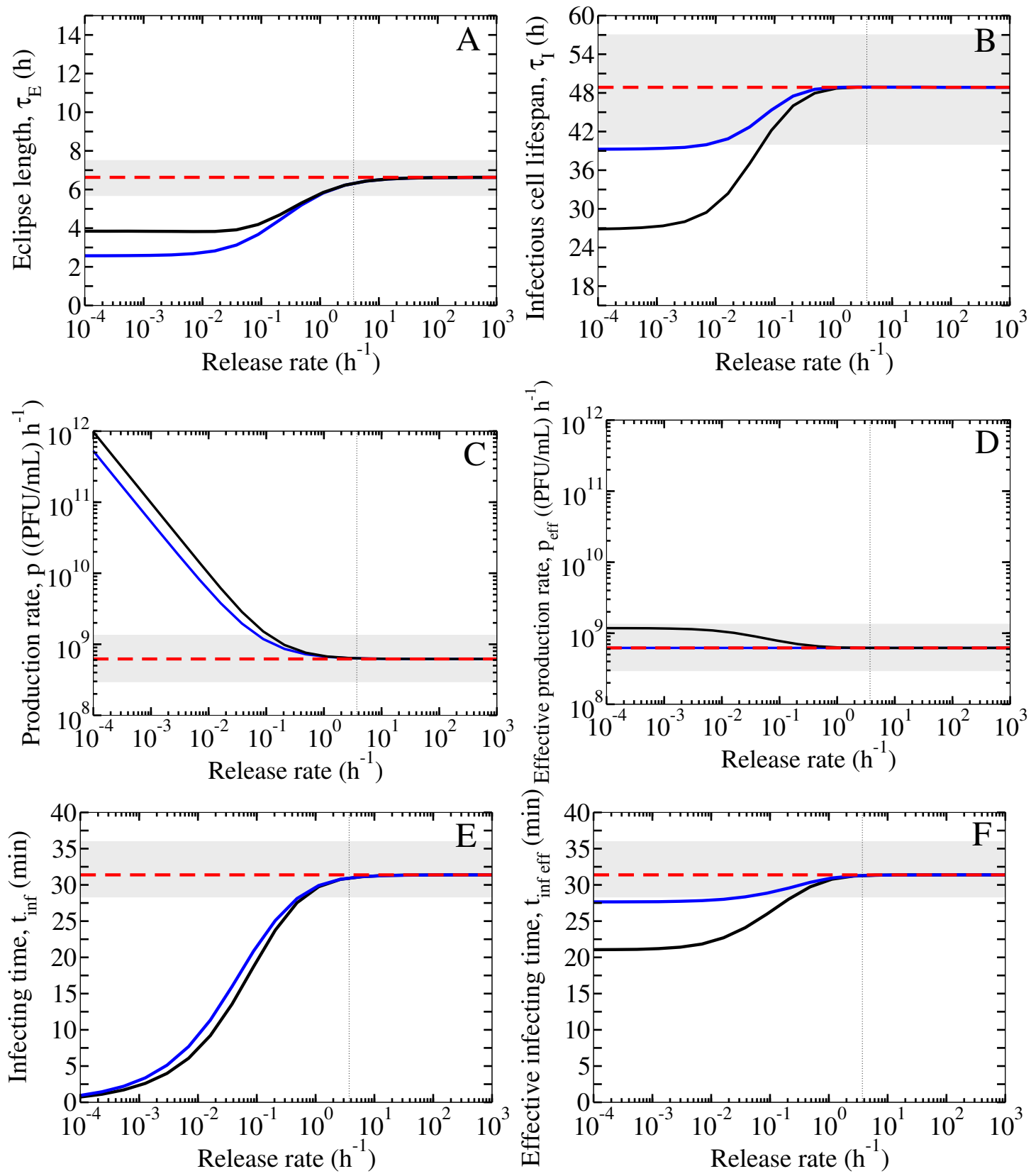

Figure 4.4: Comparing parameter estimates between the simple MM and release MM. The simple MM base parameters of an in vitro infection with the 2009 pandemic influenza A (H1N1) virus strain [85] are shown (red) with $95 \%$ confidence intervals (grey band). For the release MM, the parameter estimates obtained ( $y$-axis) as a function of the viral release rate ( $x$-axis) are shown for the case when all parameters $\left(p, \beta, c, \tau_{E}, \tau_{I}\right)$ are estimated (black), or for the case with the constraint $p_{\text {release }}=p_{\text {simple }}(r+c) / r$ (blue). The critical free virus release rate, $r_{f}=3.7 \mathrm{~h}^{-1}$, is indicated (vertical dotted line). 
Though it appears that the simple MM overestimates the eclipse length (Figure 4.4A, red vs black), this is simply a difference in interpretation of $\tau_{E}$ between these MMs.

As the virus release rate is decreased in the release MM, the production rate $(p)$ deviates from the simple MM's estimate in Figure 4.4C. Once again, parameter $p$ requires a different interpretation in each MM because it represents different processes. In the simple MM, $p$ represents the rate at which free infectious virus is produced and (instantaneously) released into the medium by an infectious cell, but in the release MM it represents the rate at which cell-associated, bound virions are produced onto the cell's surface. If the release of these cell bound virions is slow, some will lose infectivity (at rate $c$ ) before they can be released into the medium, resulting in an effective rate of free virus production into the medium which could be much less than the rate at which cell-associated bound virus are produced onto the cell's surface. It is easy to show (see Methods) that only a fraction $r /(r+c)$ of all virions produced onto the cell's surface will ultimately be released as free virions. This term also naturally appears in the equations for the basic reproductive number (Equation (4.8)) and the effective infecting time (Equation (4.9)). Therefore, it is the effective rate of free virus production, $p_{\text {eff }}=p \cdot r /(r+c)$, rather than the rate of bound virus production, $p$, in the release MM which should be compared to the free virus production rate $p$ which appears in the simple MM.

With this in mind, we repeated the fit of the release MM to the data simulated with the simple MM while fixing the effective production rate in the release MM equal to the virus production rate in the simple MM, i.e., $p_{\text {releaseMM }}=p_{\text {simplemM }}(r+c) / r=$ $\left(6.28 \times 10^{8} \mathrm{PFU} / \mathrm{mL} \cdot \mathrm{h}^{-1}\right)(r+c) / r$. From the fitted curves in Figure $4.5 \mathrm{~A}$, the SC titer recovers the familiar plateau and no longer exhibits a bump as was the case in Figure 4.3A when no constraint was applied to $p$. As the release rate decreases, the fits cannot capture the steep upslope of the SC titer. The fits performed without a constrained production rate yields the lowest Akaike information criterion $\left(\mathrm{AIC}_{\mathrm{C}}\right.$, see Methods), but does not reproduce the plateau in the $\mathrm{SC}$ assay. The $\mathrm{AIC}_{\mathrm{C}}$ is an index that ranks MMs, where the minimum score indicates the MM that gives a better fit to the data, while guarding against overfitting by imposing a penalty against MMs that have a higher number of fitted parameters. For the fits performed with a constrained production rate, a higher $\mathrm{AIC}_{\mathrm{C}}$ is obtained despite the one parameter reduction, but the plateau in the SC assay is recapitulated.

Figure 4.4 shows the parameter values predicted by the release MM under this constraint. When virus release is rapid $\left(r>r_{f}\right)$, the simple MM estimates the same parameters as predicted by the release MM. When virus release is slow $\left(r<r_{f}\right)$, the simple $\mathrm{MM}$ estimates the same effective production rate as predicted by the release MM in 

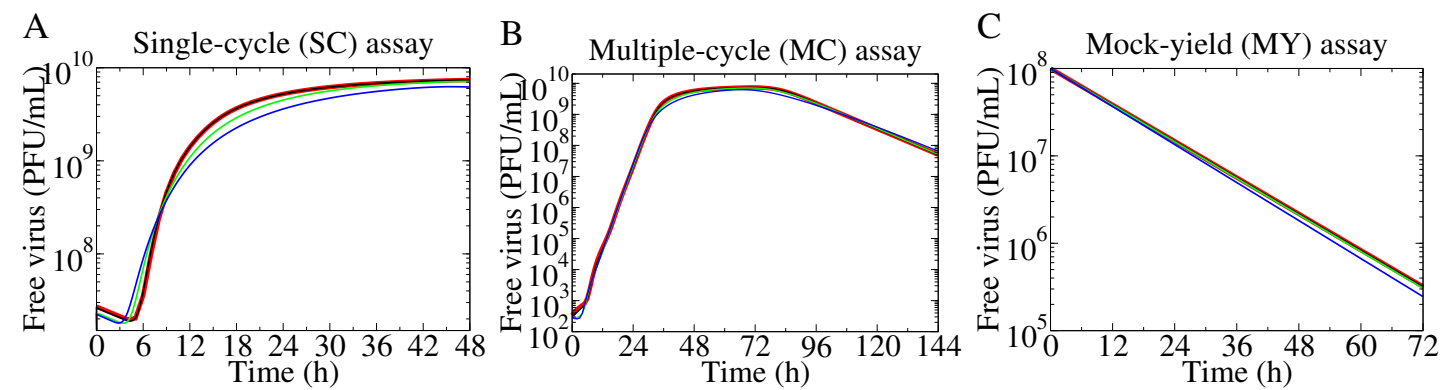

Figure 4.5: Simultaneous fits of the release MM to the simulated SC, MC, MY titers with a constraint on virus production. As in Figure 4.3, but with a constraint on the virus production rate, i.e., $p_{\text {release }}=p_{\text {simple }}(r+c) / r=$ $\left(6.28 \times 10^{8} \mathrm{PFU} / \mathrm{mL} \cdot \mathrm{h}^{-1}\right)(r+c) / r$.

either analysis. For the remaining parameters $\left(\tau_{E}, \tau_{I}, t_{\text {inf eff }}\right)$, the simple MM either: (1) overestimates them compared to the predictions by the release MM if there is no constraint on $p$; or, (2) estimates the same values as predicted by the release MM with $p$ constrained, except for the eclipse length due to the differing definitions between the two MMs.

\subsubsection{Characterizing NAI efficacy in the context of explicit vs implicit release}

We used the simple and release MMs to simulate infections in the absence (untreated) and presence (treated) of NAIs, and compared the two MM's predictions for the effect of NAIs on free virus titer. In both MMs, the NAI efficacy is expressed as a fractional inhibition, $\varepsilon$, which ranges between 0 (no inhibition) and 1 (total inhibition). In the simple MM, virus release is modelled implicitly as a part of the virus production rate $p$, so the action of NAIs is represented as inhibiting the rate of virus production, i.e., $\left(1-\varepsilon_{p}\right) p[2,17,18,32]$. In the release MM, the NAIs can be correctly implemented as inhibiting the release rate directly, i.e., $\left(1-\varepsilon_{r}\right) r$. The subscript on the symbol for efficacy denotes the infection parameter upon which the drug acts.

We simulated MC infections under NAI therapy where drug was applied at the start of the infection, at a constant efficacy of 0.99 in both MMs. If the virus release is slow $\left(r=0.01 \mathrm{~h}^{-1}\right)$, Figure $4.6 \mathrm{~A}$ shows that NAI therapy in either MM reduces and delays the free virus peak titer by the same degree. If the virus release is fast $\left(r=10 \mathrm{~h}^{-1}\right)$, the simple MM predicts that NAI therapy results in a greater reduction and delay in the free virus peak titer than that predicted by the release MM for an equal efficacy (Figure 4.6B). As a consequence, if a particular viral kinetics time course under NAI 
therapy is observed, and the efficacy is estimated, the simple MM would underestimate that efficacy. That is, if virus release is fast, the simple MM suggests that a lower efficacy of drug is required to achieve a given infection kinetics under NAI therapy, thereby also underestimating the dose required to achieve that effect.

For a comparison of the differential effect of NAIs in either MM over the entire range of release rates considered, we used a proxy to concisely represent the above information. We calculated the critical efficacy $\left(\varepsilon_{\text {crit }}\right)$ from the fitted parameters. The critical efficacy is defined as the minimum efficacy required to cause the suppression of infection, i.e., reduce $R_{0}$ below its threshold value of 1 . Figure $4.6 \mathrm{C}$ shows $\left(1-\varepsilon_{\text {crit }}\right)$ from the simple MM (Equation (4.10)) and the release MM (Equation (4.11)) as a function of the explicit release rate. At high release rates, the simple MM predicts higher values of $\left(1-\varepsilon_{\text {crit }}\right)$, indicating that a lower efficacy of NAIs is needed to achieve the total suppression of infection than predicted by the release MM. From this, we can see that the simple MM underestimates NAI efficacy if virus release is fast, where the error in estimation is largest as the virus release rate increases. Interestingly, the simple MM predicts NAI efficacies similar to those predicted by the release MM if release is slow. This is in contrast to the finding that the simple MM predicts the same estimates of the infection parameters as the release MM only if virus release is rapid.

\subsubsection{Exploring the difference between NAIs and polymerase inhibitors in the context of the release MM}

The release MM provides us with the opportunity to compare antivirals that inhibit virus production (acting on $p$ ) to those that inhibit virus release (acting on $r$ ). Figure 4.7 shows that if the true influenza A virus release rate is less than $0.01 \mathrm{~h}^{-1}$, both classes of antivirals at the same efficacy will have an equal effect on the infection. On the other hand, if the virus release rate is greater than $0.01 \mathrm{~h}^{-1}$, the release MM predicts that an antiviral inhibiting virus production requires a lower dose (is more effective) to achieve suppression of infection than an antiviral that inhibits virus release. Regardless of the true influenza A virus release rate, an antiviral acting to suppress viral replication/production is predicted to always suppress free virus titer to an extent equivalent to or greater than an antiviral inhibiting virus release. 

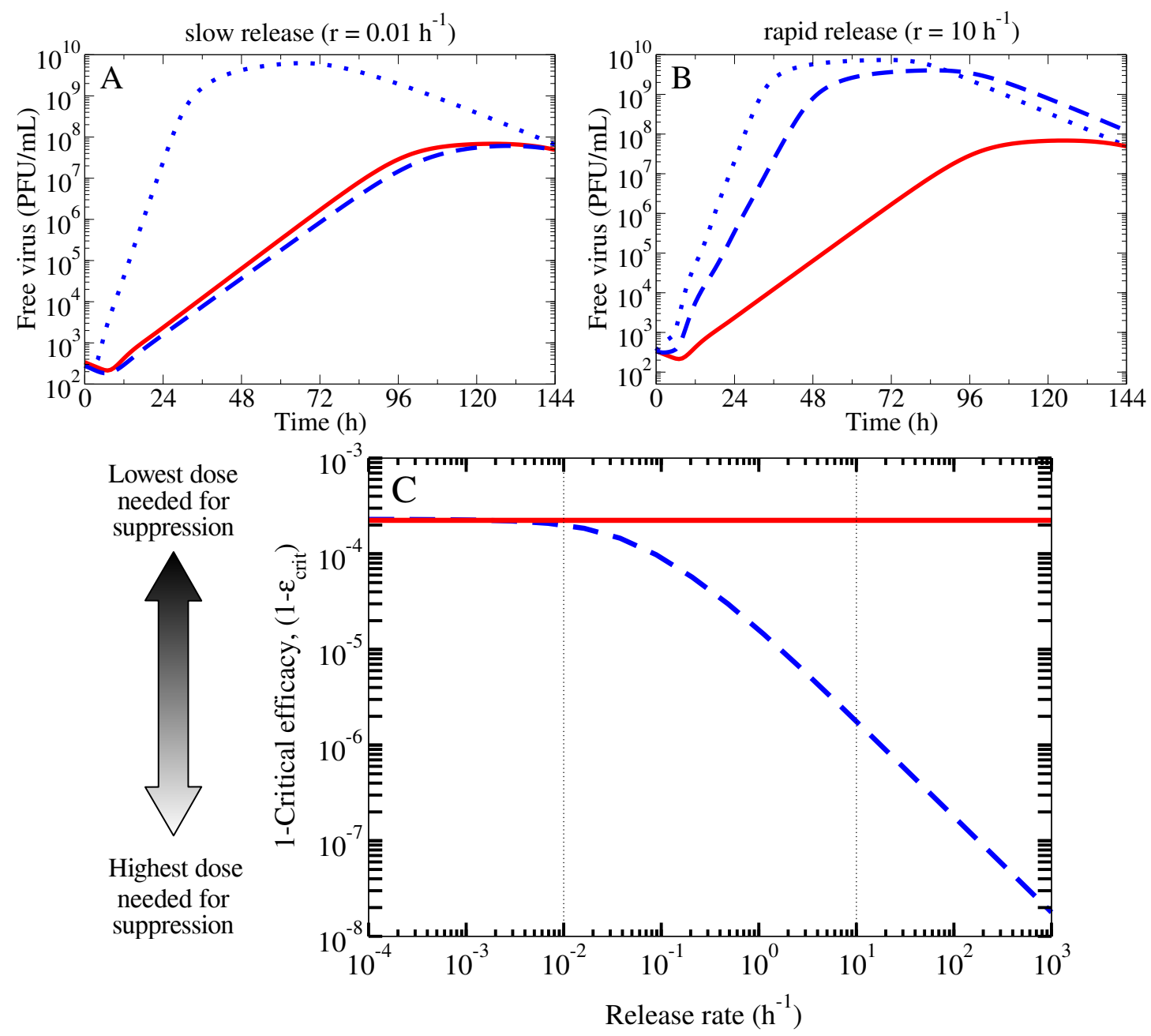

Figure 4.6: Comparing the effect of NAIs in the simple and release MMs. (A) For slow virus release $\left(r=0.01 \mathrm{~h}^{-1}\right)$, both MMs predict a similar viral kinetic time course for treatment with NAI at a constant efficacy of 0.99 , compared to the untreated infection (blue dotted line). (B) With faster virus release $\left(r=10 \mathrm{~h}^{-1}\right)$, the simple MM predicts more significant viral yield suppression than the release MM for the same NAI efficacy. (C) The $\left(1-\varepsilon_{\text {crit }}\right)$ of NAIs in the simple MM (red solid line) and the release MM (blue dashed line) is shown as a function of the release rate. The vertical dotted lines in $(\mathrm{C})$ indicate the release rates used in $(\mathrm{A})$ and $(\mathrm{B})$. 

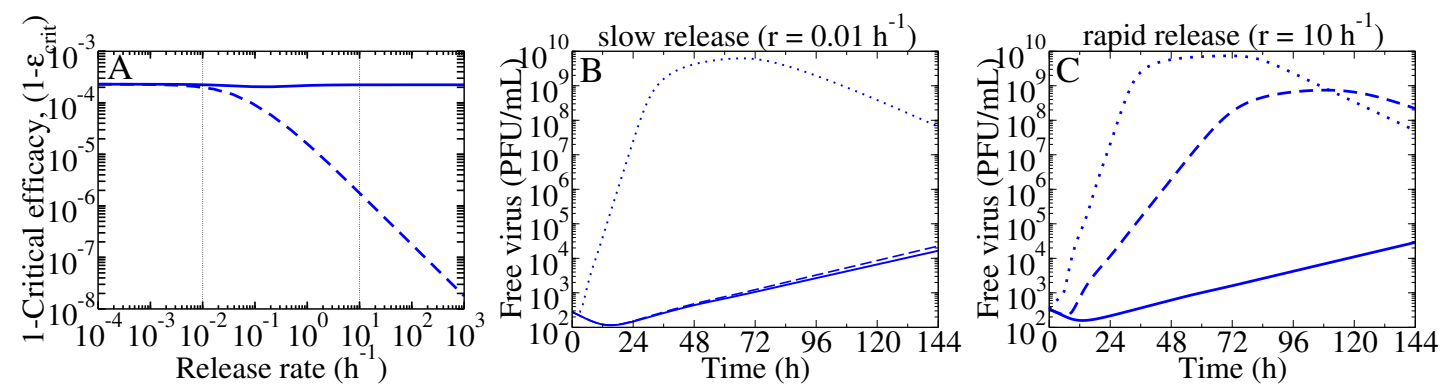

Figure 4.7: Comparing antivirals that inhibit release to antivirals that inhibit production in the release MM. (A) The $\left(1-\varepsilon_{\text {crit }}\right)$ of an antiviral that inhibits virus production $\left(\varepsilon_{p}\right.$, solid) is compared to that of an antiviral that inhibits release $\left(\varepsilon_{r}\right.$, dashed). The vertical dotted lines indicate the release rates in (B) and (C). (B) With slow release $\left(r=0.01 \mathrm{~h}^{-1}\right)$, the release MM predicts a similar viral kinetic time course for treatment with either antiviral at an efficacy of 0.999 . The untreated infection is shown (dotted). (C) With rapid release $\left(r=10 \mathrm{~h}^{-1}\right)$, the release MM predicts that an antiviral that inhibits virus production suppresses viral yield significantly more one inhibiting virus release, for the same efficacy.

\subsection{Discussion}

Mathematical models (MMs) describing the kinetics of influenza A virus infections in vitro and in vivo typically represent the release of cell-bound progeny virions from the surface of productively infected cell implicitly. Specifically, the rate of virus release gets incorporated into aggregate, generic parameters which stand in for the combined kinetics of virus replication, assembly, budding, and release. Importantly, when these simple MMs are used to characterize treatment of influenza A virus infections with NAIs $[2,17,18,32]$, they represent the action of NAIs as suppressing virus production, or rather suppressing the combined kinetics of replication, assembly, budding and release embodied in the simple MM's parameter designated as the rate of free virus production.

Herein we developed a variant of the simple MM, hereafter the release MM, that includes an explicit description of virus release. The release MM accounts for two distinct populations of infectious virus, bound and free virions, with cell-associated, bound virions being released into the medium as free virions at release rate $r$. Unfortunately, the rate at which influenza A virus progeny is released from the surface of the cell that produces them is not well-known. Using the release MM, we explored a wide range of virus release rates to assess the impact of this additional, unknown MM parameter on infection parameter estimates, and in particular the estimation of NAI efficacy, compared to those obtained with the simple MM. 
Mathematically, in the absence of antiviral therapy, the release MM reduces to the simple MM if the virus release rate is sufficiently large. Our work shows that for realistic parameter values in the context of an influenza A virus infection in vitro, this occurs when the virus release rate is greater than $\sim 4 \mathrm{~h}^{-1}$. If virus release is more efficient than this, release does not significantly affect infection kinetics or free virus yield, making the simple MM a valid approximation of the release MM. If virus release is less efficient than this, it will affect infection kinetics and free virus yield. In fact, if virus release is slower than $\sim 0.1 \mathrm{~h}^{-1}$, the two MMs statistically significantly disagree in their estimates of some infection parameters, specifically the virus production rate, the eclipse length, and the lifespan of infectious cells (i.e., the duration of virus production). This is because although the simple and release MMs share most of the same structure, the implementation of implicit versus explicit virus release imparts onto each MM a different interpretation of the infection parameters they have in common.

Biologically, if the release of virions into the medium is slow such that the release rather than the production of virus is a kinetic bottleneck, a portion of the bound virions produced onto the cell's surface will lose infectivity before they can be released as free virions into the medium. For the release MM, we show that only a fraction $r /(r+c)$ of the bound virions produced onto the cell's surface are ultimately released into the medium as free virions, wherein $r$ is the virus release rate and $c$ is the rate at which virions lose infectivity. If $r$ is large relative to $c, r /(r+c) \approx 1$, and all produced virions are released as free virions. In fact, we found that the free virus production rate $(p)$ estimated by the simple MM agrees with the effective free virus production rate $(p \cdot r /(r+c))$ estimated by the release MM. Furthermore, when we repeated parameter estimation while specifically imposing the constraint that $p_{\text {simple }}=p_{\text {release }} r /(r+c)$, the two MMs agreed in their estimation of all parameters except for the length of the eclipse phase. While the eclipse phase length in the simple MM measures the delay from the infection of a cell until free virus release, in the release MM it measures the time elapsed between infection of the cell and the appearance of progeny virions bound to the infected cell's surface, specifically excluding the time required for their release as free virus. For a given experimentally observed delay in the appearance of free virions, the simple MM can only ascribe it to the eclipse length whereas the release MM must distribute its duration between the eclipse length and release rate. This should be kept in mind when interpreting estimates of the eclipse phase length obtained using the simple MM.

When it comes to representing the mode of action of NAIs, the simple MM is restricted to inhibiting the aggregate free virus production rate, whereas the explicit MM can specifically inhibit the rate of virus release. If virus release is slow $(r<c)$ such that 
release rather than production limits free virus yield, the two MMs predict the same infection kinetics for the same NAI efficacy. In the release MM, if virus release is rapid such that virus production rather than release is the bottleneck, imposing the limit on free virus yield $(r \gg c)$, even a large reduction in the release rate has a modest impact on virus yield until it is reduced to the point where $r \approx c$. For this reason, if virus release is rapid, NAIs in the release MM have a weaker suppressive effect than in the simple MM for the same efficacy. In other words, for a given experimentally observed reduction in free viral yield, the simple MM will underestimate the true NAI efficacy required to effect this reduction. This error increases with increasing virus release rates. Underestimating antiviral efficacy in turn, leads to underestimating the NAI dosage required to achieve a specific therapeutic outcome, e.g., suppression of infection. Underdosing prolongs the overall duration of infection and the number of genomes produced, which could increase the likelihood of developing resistance to NAIs. In Japan, NAI-resistant A/H3N2 strains were detected from children who received oseltamivir, and underdosing had been thought to be a contributing factor [56, 62].

Our results demonstrate the need to estimate the rate of influenza A virus release in order to correctly interpret results obtained from the simple MM. However, directly measuring the virus release rate is difficult for a number of reasons. An uninfected cell will express a number of sialic acid receptors on its surface. After successful infection by an influenza A virus, virus replication gets underway and $\sim 1 \mathrm{~h}-3 \mathrm{~h}$ post-infection, the cell begins to express increasing levels of viral NA on its surface $[12,58]$. These surface viral NAs proceed to cleave more and more of the cell's sialic acid receptors as time elapses. As such, the sialic acid receptor landscape seen by progeny virions budding onto the cell's surface is much less dense than than that seen by infecting influenza A virions entering an uninfected cell. For this reason, detachment rates of influenza A virus measured in studies using uninfected cells (where endocytosis and infection are blocked) cannot be thought of as estimates of the virus release rates, but could possibly be providing a lower bound for that estimate. For example, Nunes-Correia et al. [79] found detachment rates of $0.36 \mathrm{~h}^{-1}-11 \mathrm{~h}^{-1}$ for influenza A PR8 (H1N1) virus interacting with uninfected MDCK cells. Since there are more receptors on uninfected cells, we expect that the true virus release rate would be greater than that found by Nunes-Correia et al. However, the study by Nunes-Correia et al. was performed on MDCK cells which express far fewer $\alpha-2,6$ cell receptors than that found on the surface of epithelial cells lining the human respiratory tract (RT) [81]. Due to this lower level of $\alpha-2,6$ on MDCK cells, these rates likely overestimate the biologically relevant detachment rates and therefore cannot be thought of as a true lower bound. In [31], other key studies of influenza A virus attachment and 
detachment from uninfected cells were highlighted, with detachment rates estimates as low as $0.029 \mathrm{~h}^{-1}$ and as high as $11 \mathrm{~h}^{-1}$. However, these were estimated with different influenza A viruses interacting in non-cell systems (e.g., influenza A PR8 (H1N1) virus interacting with bovine brain lipid membrane, or influenza A (H3N2) and duck influenza A (H5N3) virus strains binding to gangliosides) which might not reflect the cell receptor composition of an infected human RT epithelial cell.

Our release MM predicts that at equal efficacy, treatment with an antiviral against virus production suppresses free virus yield to a greater extent than an antiviral against release if the release rate is greater than $0.01 \mathrm{~h}^{-1}$. This is consistent with the observation that favipiravir, a new antiviral which inhibits viral polymerase, is more potent and effective than NAIs [101] (refer to [27] for a review). Therefore, it is possible that influenza A virus strains that are more strongly suppressed by favipiravir than NAIs have a release rate greater than $\sim 0.01 \mathrm{~h}^{-1}$. In our view, the best way to obtain an estimated lower bound for the release rates, would be to repeat the attachment/detachment study performed by Nunes-Correia et al. using MDCK $\alpha 2,6$ cells which express $\alpha-2,6$ sialic acid cell receptors at levels similar to those found on human RT epithelial cells.

In our work, we assume that the virus release rate is constant throughout the entirety of the cell's infectious phase. As discussed above, the amount of cell surface NA increases and the number of cell receptors decreases as the infection progresses, such that the rate of virus release should in fact depend on the time elapsed since the cell became infected. One possible MM extension is the integration of an age of infection-dependent release rate, where a simple step function could switch from a low to high release rate as the infection progresses. Without appropriate quantitative data, however, there is little to justify the added complexity to the MM.

In conclusion, we have highlighted the importance of quantifying the influenza A virus release rate and the implications of an implicit representation of virus release in the simple MM. In future analyses of virus replication kinetics, it would be desirable to design an additional in vitro assay, to expand the current suite of assays (i.e., MC, SC, MY), which could provide an accurate, independent estimate of the virus release rate. It remains to be determined whether or not viral release acts as a kinetic bottleneck in influenza A virus replication. 


\subsection{Methods}

\subsubsection{Mathematical models of influenza A viral infection kinetics}

Influenza A virus infections in vitro were numerically simulated with a multi-compartment ordinary differential equation (ODE) model as previously described and validated in [81, 85, 98]. Throughout, we refer to it as the simple MM in order to distinguish it from a variant we call the release MM. The release MM shares almost all equations with the simple MM, but possesses one additional parameter, the virus release rate $r$, and one additional equation to distinguish cell-associated bound virus from released free virus. The equations common to both MMs describes the flow of cells through the various phases of infection as follows

$$
\begin{aligned}
\frac{\mathrm{d} T}{\mathrm{~d} t} & =-\beta T V \\
\frac{\mathrm{d} E_{1}}{\mathrm{~d} t} & =\beta T V-\frac{n_{E}}{\tau_{E}} E_{1} \\
\frac{\mathrm{d} E_{i}}{\mathrm{~d} t} & =\frac{n_{E}}{\tau_{E}} E_{i-1}-\frac{n_{E}}{\tau_{E}} E_{i} \text { for } i=\left(2, \ldots, n_{E}\right) \\
\frac{\mathrm{d} I_{1}}{\mathrm{~d} t} & =\frac{n_{E}}{\tau_{E}} E_{n_{E}}-\frac{n_{I}}{\tau_{I}} I_{1} \\
\frac{\mathrm{d} I_{j}}{\mathrm{~d} t} & =\frac{n_{I}}{\tau_{I}} I_{j-1}-\frac{n_{I}}{\tau_{I}} I_{j} \text { for } j=\left(2, \ldots, n_{I}\right)
\end{aligned}
$$

where fractional $(\in[0,1])$ populations of uninfected target cells, $T$, are infected at infection rate $\beta$ times the concentration of free, infectious virus, $V$ (in PFU/mL). Newly infected cells enter the eclipse phase, $E_{i=1, \ldots, n_{E}}$, and transition into the infectious phase, $I_{j=1, \ldots, n_{I}}$, after an average time $\tau_{E}$ and $\tau_{I}$ have elapsed, respectively. The eclipse (or infectious) phase is divided into $n_{E}$ (or $n_{I}$ ) compartments such that the time spent by cells in the phase follows an Erlang distribution [37, 38, 85].

The two MMs differ only in their equations for infectious virus, wherein the release MM includes one additional variable and one new parameter to explicitly account for the release of cell-bound virus as free virus by the action of viral neuraminidase (NA)

Simple MM

$$
\begin{aligned}
\frac{\mathrm{d} V_{\mathrm{b}}}{\mathrm{d} t} & =p \sum_{i=1}^{n_{I}} I_{i}-c V_{\mathrm{b}}-r V_{\mathrm{b}} \\
\frac{\mathrm{d} V}{\mathrm{~d} t}=p \sum_{i=1}^{n_{I}} I_{i}-c V & \frac{\mathrm{d} V}{\mathrm{~d} t}=r V_{\mathrm{b}}-c V .
\end{aligned}
$$


In the simple MM (without explicit viral release), free infectious virus $V$ is produced directly into the medium by infectious cells $(I)$ at a rate $p$, and lose infectivity at rate $c$. In the release MM, cell-bound virus $V_{b}$ is produced onto the surface of infectious cells at a rate $p$, and also lose infectivity at rate $c$ while bound to the cell. The infectious bound virus is released from the cell surface at rate $r$ and enters the medium as free virus, which also lose infectivity at rate $c$. The release MM distinguishes virus release from any other processes that are encompassed by the virus production rate. Our base parameter values for the simple MM are listed in Table 4.1, and were taken from [85] where the kinetics of an in vitro infection with the 2009 pandemic influenza A/Québec/144147/09 (H1N1) virus strain was analyzed.

Table 4.1: Base parameter values and $\mathbf{9 5 \%}$ confidence intervals for infection with an influenza A (H1N1) pandemic strain [85].

\begin{tabular}{lc} 
Parameter, symbol & Base value $[95 \% \mathrm{CI}]$ \\
\hline Virus production rate, $p$ & $6.21 \times 10^{8}[2.96-13.4](\mathrm{PFU} / \mathrm{mL}) \cdot \mathrm{h}^{-1}$ \\
Virus infection rate, $\beta$ & $1.18 \times 10^{-8}[0.463-3.03](\mathrm{PFU} / \mathrm{mL})^{-1} \cdot \mathrm{h}^{-1}$ \\
Rate of loss of infectious virus, $c$ & $7.98 \times 10^{-2}[5.05-11.1] \mathrm{h}^{-1}$ \\
Infected cell eclipse length, $\tau_{E}$ & $6.63[5.75-7.44] \mathrm{h}$ \\
Infected cell infectious cell lifespan, $\tau_{I}$ & $48.9[40.0-57.0] \mathrm{h}$ \\
Number of eclipse compartments, $n_{E}$ & 30 \\
Number of infectious compartments, $n_{I}$ & 100 \\
\hline
\end{tabular}

Two important secondary parameters, the basic reproductive number $\left(R_{0}\right)$ and the infecting time $\left(t_{\text {inf }}\right)$, can be derived from the MM parameters. The infecting time represents the time it takes for one infectious, virus-producing cell to infect one other cell in a fully susceptible population of cells. It has been described in [37, 38, 39] and is given by

$$
t_{\mathrm{inf}}=\sqrt{\frac{2}{p \beta}} .
$$

The infecting time is reported in lieu of parameters that are measured in plaque-forming units, such as $p$ and $\beta$, because of the undetermined, relative relationship between infectious virus and experimental measures of virus infectivity such as $50 \%$ infectious dose $\left(\right.$ TCID $\left._{50}, \mathrm{CCID}_{50}\right)$ or plaque-forming unit (PFU) $[3,4]$.

The basic reproductive number is another commonly reported quantity and it represents the number of secondary infections caused by one infectious cell in a population of fully susceptible cells. If $R_{0}$ is less than the threshold value of 1 , the spread of infection 
is suppressed. The basic reproductive number for each MM is given by

$$
\begin{array}{lr}
\text { Simple MM } & \text { Release MM } \\
R_{0}=\frac{p \beta \tau_{I}}{c} & R_{0}=\frac{p \beta \tau_{I}}{c}\left[\frac{r}{r+c}\right] .
\end{array}
$$

\subsubsection{The effective production rate in the release MM}

Assuming that the fraction of productively infectious cells is unchanged over a certain timescale of interest $(I=\bar{I})$, Eqn. (4.6) for bound virus in the release MM becomes

$$
\frac{\mathrm{d} V_{\mathrm{b}}}{\mathrm{d} t}=p \bar{I}-(r+c) V_{\mathrm{b}} .
$$

Further assuming that no bound virus is initially present, integrating the above equation yields:

$$
V_{\mathrm{b}}(t)=\frac{p \bar{I}}{r+c}\left[1-\mathrm{e}^{-(r+c) t}\right] .
$$

Substitution of $V_{\mathrm{b}}(t)$ into the differential equation describing free virus yields:

$$
\frac{\mathrm{d} V}{\mathrm{~d} t}=\underbrace{\frac{r}{r+c}}_{p_{\mathrm{eff}}} p \bar{I}\left[1-\mathrm{e}^{-(r+c) t}\right]-c V
$$

where the correction factor $\frac{r}{r+c}$ on the virus production rate accounts for bound virus which loses infectivity before it can be released. Thus, the effective production rate in the release $\mathrm{MM}$ is given by $p_{\text {eff }}=p \frac{r}{r+c}$.

We use the effective production rate to correct the expression of the infecting time. This corrected parameter is called the effective infecting time,

$$
t_{\text {inf eff }}=\sqrt{\frac{2}{p_{\text {eff }} \beta}}=\sqrt{\frac{2}{p \beta} \frac{(r+c)}{r}} .
$$

Such a correction is necessary since the infecting time $\left(t_{\text {inf }}\right)$ will deviate from the intended physical meaning for release rates that are less than $0.1 \mathrm{~h}^{-1}$. This is because the expression for $t_{\text {inf }}$ was originally derived under the assumption that the rate of loss of virion (approximately $0.1 \mathrm{~h}^{-1}$ ) could be neglected. 


\subsubsection{Modelling antiviral effect}

When infections are treated with a neuraminidase inhibitor (NAI), the drug efficacy $\varepsilon$ is applied to either the production rate in the simple MM, as $\left(1-\varepsilon_{p}\right) p$, or to the release rate in the release $\mathrm{MM}$, as $\left(1-\varepsilon_{r}\right) r$. The efficacy $\left(\varepsilon_{p}\right.$ or $\left.\varepsilon_{r}\right)$ takes on values between 0 (no efficacy) and 1 (maximum efficacy), where a more efficacious drug leads to greater reduction of either the virus production or release rate. Treatment is always applied at the start of infection $(t=0 \mathrm{~h})$.

In each MM, treatment with NAIs is given by:

Simple MM

$$
\begin{aligned}
\frac{\mathrm{d} V_{\mathrm{b}}}{\mathrm{d} t} & =p \sum_{i=1}^{n_{I}} I_{i}-c V_{\mathrm{b}}-\left(1-\varepsilon_{r}\right) r V_{\mathrm{b}} \\
\frac{\mathrm{d} V}{\mathrm{~d} t}=\left(1-\varepsilon_{p}\right) p \sum_{i=1}^{n_{I}} I_{i}-c V & \frac{\mathrm{d} V}{\mathrm{~d} t}=\left(1-\varepsilon_{r}\right) r V_{\mathrm{b}}-c V
\end{aligned}
$$$$
\text { Release MM }
$$

Additionally, the release MM allows for treatment with an antiviral which targets the virus production rate, separate from that which targets the virus release rate. In the release MM, treatment with an antiviral which blocks virus production is given by:

Release MM

$$
\frac{\mathrm{d} V_{\mathrm{b}}}{\mathrm{d} t}=\left(1-\varepsilon_{p}\right) p \sum_{i=1}^{n_{I}} I_{i}-c V_{\mathrm{b}}-r V_{\mathrm{b}} .
$$

We define the critical efficacy $\left(\varepsilon_{\text {crit }}\right)$ as the minimum efficacy required to reduce the basic reproductive number below 1 . Re-arranging the above when $R_{0}=1$ yields the following expressions for critical efficacies in the simple MM

$$
\varepsilon_{p \text { crit }}=1-\frac{c}{p \beta \tau_{I}}
$$

and in the release MM

$$
\begin{gathered}
\varepsilon_{p \text { crit }}=1-\frac{c}{p \beta \tau_{I}} \frac{(c+r)}{r} \\
\varepsilon_{r \text { crit }}=1-\frac{1}{\left[\frac{p \beta \tau_{I}}{c}-1\right] \frac{c}{r} .}
\end{gathered}
$$




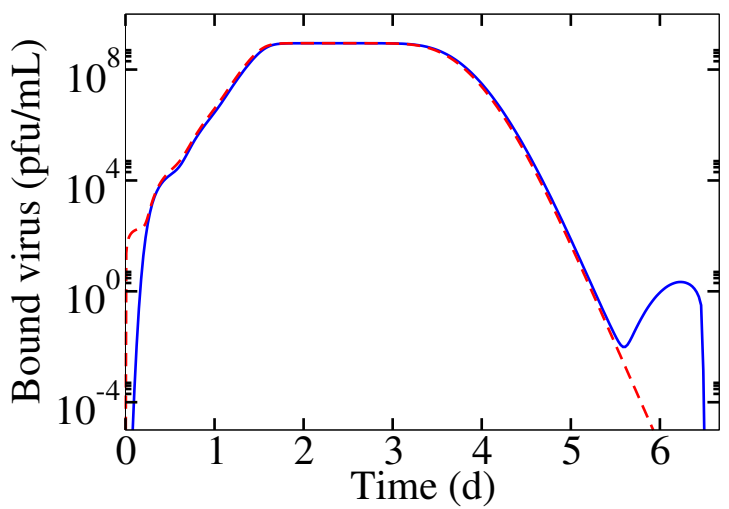

Figure 4.8: Instabilities in numerically solving model ordinary differential equations (ODE). Numerically solving ODEs in Equations (4.1)-(4.7) (blue) results in instabilities, however solving transformed Equations (4.13)-(4.19) (red) resolves the issue.

\subsubsection{Numerically solving transformed equations}

Numerically solving the MM in the form of Equations (4.1)-(4.7) with the 1sode solver in Octave 3.8.1 suffered from instabilities (Figure 4.8). The instabilities are due, in part, to finite machine precision as well as stiff MM equations, which solvers cannot overcome. Such instabilities can be observed even when attempting to numerically solve an equation for a simple exponential decay.

One way to resolve issues with very small numerical values and the accumulation of errors, is to set the value to zero when it becomes physically unreasonable. However, we chose another way to resolve this issue, where a change of variables was done to transform Equations (4.1)-(4.7). For example, Equation (4.1) was transformed by introducing variables $L_{V}=\ln \left(\frac{V}{V_{\text {res }}}\right)$ and $L_{T}=\ln \left(\frac{T}{N}\right)$ where $V_{\text {res }}$ and $N$ were rescaling factors required to maintain a dimensionless argument within the natural logarithm. For example, Equation (4.1) becomes

$$
\frac{\mathrm{d} L_{T}}{\mathrm{~d} t}=\frac{\mathrm{d} L_{T}}{\mathrm{~d} T} \frac{\mathrm{d} T}{\mathrm{~d} t}=-\frac{\mathrm{e}^{-L_{T}}}{N} \beta T V=-\frac{\mathrm{e}^{-L_{T}}}{N} \beta\left(N \mathrm{e}^{L_{T}}\right)\left(V_{\mathrm{res}} \mathrm{e}^{L_{V}}\right) .
$$


The resulting full set of transformed equations is given by

$$
\begin{aligned}
\frac{\mathrm{d} L_{T}}{\mathrm{~d} t} & =-\beta V_{\mathrm{res}} \mathrm{e}^{L_{V}} \\
\frac{\mathrm{d} L_{E_{1}}}{\mathrm{~d} t} & =\beta V_{\mathrm{res}} \mathrm{e}^{L_{T}+L_{V}-L_{E_{1}}}-\frac{n_{E}}{\tau_{E}} \\
\frac{\mathrm{d} L_{E_{i}}}{\mathrm{~d} t} & =\frac{n_{E}}{\tau_{E}} \mathrm{e}^{L_{E_{i-1}}-L_{E_{i}}}-\frac{n_{E}}{\tau_{E}} \\
\frac{\mathrm{d} L_{I_{1}}}{\mathrm{~d} t} & =\frac{n_{E}}{\tau_{E}} \mathrm{e}^{L_{E_{n_{E}}}-L_{I_{1}}}-\frac{n_{I}}{\tau_{I}} \\
\frac{\mathrm{d} L_{I_{i}}}{\mathrm{~d} t} & =\frac{n_{I}}{\tau_{I}} \mathrm{e}^{L_{I_{i-1}}-L_{I_{i}}}-\frac{n_{I}}{\tau_{I}}
\end{aligned}
$$

Simple MM

$$
\begin{aligned}
& \frac{\mathrm{d} L_{V}}{\mathrm{~d} t}=p \frac{N}{V_{\mathrm{res}}} \mathrm{e}^{-L_{V}} \sum_{i=1}^{n_{I}} \mathrm{e}^{L_{I_{i}}}-c \quad \frac{\mathrm{d} L_{V_{b}}}{\mathrm{~d} t}=p \frac{N}{V_{\mathrm{res}}} \mathrm{e}^{-L_{V_{b}}} \sum_{i=1}^{n_{I}} \mathrm{e}^{L_{I_{i}}}-c-r \\
& \frac{\mathrm{d} L_{V}}{\mathrm{~d} t}=r \mathrm{e}^{L_{V_{b}}-L_{V}}-c
\end{aligned}
$$

Figure 4.8 shows that a change of variables resolved the errors in numerically solving the system of equations. The solution now correctly reflects that late in the infection the infectious virus follows a pure exponential decay (since all cells have died), instead of exhibiting a secondary peak of infectious virus (i.e., the instability). Furthermore, when both MMs are approximately mathematically equivalent, the solution from the transformed equations (for $r=1000 \mathrm{~h}^{-1}$ ) was verified to match the solution from the simple MM, with base parameter values used in both.

\subsubsection{Simulation of infections}

\section{Multiple-cycle, single-cycle and mock-yield simulated data}

The multiple-cycle (MC), single-cycle (SC), and mock-yield (MY) in vitro assays in Figures 4.2, 4.3, 4.5 (red) were simulated with the simple MM, using the base parameters in Table 4.1. The MC and SC assays were initiated with a population of fully susceptible cells $(T=1)$ that were infected with concentrations of the initial inoculum according to $V_{0}=\frac{c}{\beta}(\mathrm{MOI})$, where the MOIs were $5 \times 10^{-5} \mathrm{PFU} /$ cell and $4 \mathrm{PFU} /$ cell, respectively. The MY assay was initiated with an initial virus inoculum of $V_{0}=10^{8} \mathrm{PFU} /$ cell in the absence of cells $(T=0)$. The free virus titer from this suite of in vitro assays served as our simulated data with which the release MM was fitted. 
In Figure 4.2 (black), infections were simulated with the release MM where the initial conditions and parameters were identical to the MC simulation in the simple MM, except that the release rate was varied as indicated.

\section{Fitting the release MM to the simple MM-simulated data}

Simultaneous fits of the release MM to the SC, MC, and MY viral yield simulated datasets were performed with the Nelder-Mead minimization method (nelder_mead_min from version 1.5.2 of the optim package in Octave 4.0.3). The fits minimized the combined sum-of-squared residuals per point $(\mathrm{SSR} / \mathrm{pt})$ :

$$
\text { combined SSR } / \mathrm{pt}=\mathrm{SSR} / \mathrm{pt}_{\mathrm{SC}} N_{\mathrm{SC}}+\mathrm{SSR} / \mathrm{pt}_{\mathrm{MC}} N_{\mathrm{MC}}+\mathrm{SSR} / \mathrm{pt}_{\mathrm{MY}} N_{\mathrm{MY}}
$$

where SSR $/ \mathrm{pt}=\frac{1}{N} \sum_{i=1}^{N} \log _{10}\left(\frac{V_{i}^{\text {data }}}{V_{i}^{\text {expMM }}}\right)^{2}$ is computed for each assay, and $i$ is the number of points of simulated free virus from the simple MM $\left(V_{i}^{\text {data }}\right)$ and release MM $\left(V_{i}^{\text {expMM }}\right)$. Each term is weighted by the number of experimental measurements typically made when collecting free virus titer in each assay $\left(N_{\mathrm{SC}}=17, N_{\mathrm{MC}}=12, N_{\mathrm{MY}}=4\right)$ [98].

In each fit, the release rate was fixed in the release MM, while the parameters $p, \beta, c, \tau_{E}, \tau_{I}$ were fitted. The remaining parameters $n_{E}, n_{I}$ were kept at their base values (Table 4.1). The initial conditions for each assay were as described in Section 4.4.5. In total, multiple fits were performed for release rates in the range $10^{-4} \mathrm{~h}^{-1}-10^{3} \mathrm{~h}^{-1}$. The resultant sets of fitted parameters are shown in Figure 4.4 (black) and Appendix Figure B.4. To verify our results, we repeated the least-square fitting extensively with alternating Levenberg-Marquardt (4 repetitions) and Nelder-Mead Simplex (1 repetition) methods. In one case $\left(r=100 \mathrm{~h}^{-1}\right)$, we used a Markov chain Monte Carlo method to extract the posterior probability density distribution of model parameters. The final parameters extracted remained the same as reported.

In another analysis, a constraint was imposed on the release MM's virus production rate $p_{\text {release }}=p_{\text {simple }} \frac{r+c}{r}$, where $p_{\text {simple }}=6.21 \times 10^{8}(\mathrm{PFU} / \mathrm{mL}) \cdot \mathrm{h}^{-1}$, enforcing the effective production rate in the release $\mathrm{MM}$ to be equal to the production rate in the simple MM. In this case, only four parameters were fitted (i.e., $\beta, c, \tau_{E}, \tau_{I}$ ) for each value of the release rate that was explored. The resultant sets of fitted parameters are shown in Figure 4.4 (blue) and Appendix Figure B.4.

In Figure 4.9, we compared the analyses by computing the Akaike's information criterion (corrected for small sample sizes) according to the expression given by [4], and 


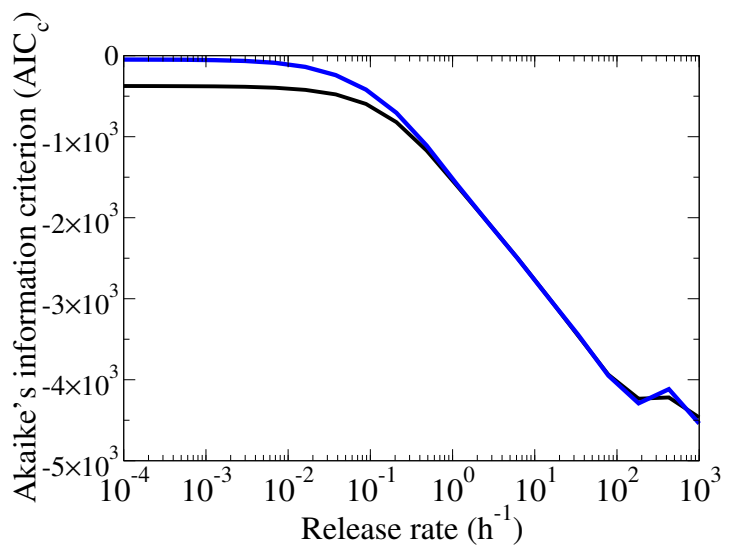

Figure 4.9: Comparing the $\mathbf{A I C}_{\mathbf{C}}$ of release $\mathbf{M M}$ fits. The corrected Akaike information criterion $\left(\mathrm{AIC}_{\mathrm{C}}\right)$ is shown for fits to the release MM with a constraint on the virus production rate $\left(N_{\text {par }}=4\right.$, blue $)$, and without a constraint $\left(N_{\text {par }}=5\right.$, black $)$.

based on [9]:

$$
A I C_{\mathrm{C}}=N_{\mathrm{pts}} \ln \left(\frac{S S R}{N}\right)+\frac{2\left(N_{\mathrm{par}}+1\right) N_{\mathrm{pts}}}{N_{\mathrm{pts}}-N_{\mathrm{par}}-2}
$$

where $\frac{S S R}{N}$ is the combined SSR/pt from the fit, $N_{\mathrm{pts}}=307$ is the number of simulated data points and $N_{\text {par }}$ is the number of parameters fitted. In the analysis without a constraint on the virus production rate $N_{\text {par }}=5$, and in the analysis with the constraint $N_{\text {par }}=4$.

\subsubsection{Treatment with antivirals}

To illustrate that NAIs applied at identical efficacies in both MMs had a different effect on the free virus titer, we showed a MC infection in the absence of NAIs (untreated) and in the presence of NAIs (treated), as simulated by either MM in Figure 4.6A, B. The untreated and treated free virus titer were simulated in the simple MM using the base parameters (Table 4.1), where the treated infection received NAIs at the start of infection that were applied at an efficacy of $\varepsilon_{p}=0.99$. With the constraint on virus production (Section 4.4.5), the release MM was fitted to the simple MM's untreated free virus titer, and recapitulated these kinetics with a different set of parameters for each chosen release rate, $r=0.01 \mathrm{~h}^{-1}, 10 \mathrm{~h}^{-1}$. Using these sets of parameters, the treated infections in the release MM were simulated with NAIs applied at an efficacy of $\varepsilon_{r}=0.99$ at the start of infection.

In Figure 4.6C (blue), the critical efficacy of NAIs as a function of the release rate was computed (Equation (4.12)) from the sets of fitted parameters that were obtained 
from the release MM when a constraint was applied to the virus production rate. For comparison, the critical efficacy of NAIs from the simple MM (Equation (4.10)) was computed from the base parameters (Table 4.1), and was shown as a single value (straight line), independent of the release rate in Figure 4.6C (red).

In Figure 4.7A, the critical efficacy of antivirals against release (dashed) or production (solid) were computed with Equation (4.12) and Equation (4.11), respectively, using the same sets of fitted parameters that were determined with the release MM. To illustrate that the impact of antivirals which inhibit viral release differed from antivirals which inhibit virus production, untreated and treated MC infections were simulated in the release MM. For example, Figure 4.7B shows an untreated infection (blue dotted) that was simulated with the set of fitted parameters that was determined when the release rate was fixed to $0.01 \mathrm{~h}^{-1}$. The corresponding treated infections were simulated by applying an antiviral against production (solid) or release (dashed) at an efficacy of 0.999 at the start of infection. Figure $4.7 \mathrm{C}$ is the same, but a higher release rate $\left(10 \mathrm{~h}^{-1}\right)$ was used to simulate the infections. 


\section{Acknowledgements}

The authors wish to acknowledge Diana Schwendener Forkel for providing the original artwork which was adapted for the purposes of, and which now appears in, Figure 4.1.

\section{Funding}

This work was supported in part by Discovery Grant 355837-2013 from the Natural Sciences and Engineering Research Council of Canada (www.nserc-crsng.gc.ca), and by Early Researcher Award ER13-09-040 from the Ministry of Research and Innovation and Science of the Government of Ontario (www.ontario.ca/page/early-researcher-awards), both awarded to CAAB, and by the Interdisciplinary Theoretical and Mathematical Sciences (iTHES, ithes.riken.jp; iTHEMS, ithems.riken.jp) research programmes at RIKEN (CAAB). Additional support in the form of an Ontario Graduate Scholarship from the Government of Ontario (www.osap.gov.on.ca) (LEL), a Michael Smith Foreign Study Supplement and a Doctoral Canadian Graduate Scholarship (CGS-D) from the Natural Sciences and Engineering Research Council of Canada (www.nserc-crsng.gc.ca) were awarded to LEL. The funders had no role in study design, data collection and analysis, decision to publish, or preparation of the manuscript. 


\section{Chapter 5}

\section{Conclusion}

The overall aim of this study was to re-examine and refine quantitative methods based on in vitro assays of influenza A virus infection, with the help of MMs. Based on our MMs we provided two significant, new pieces of knowledge that should be taken into account when applying such methods. First, we showed that the DIP counting in vitro assay produces valid results only if certain criteria, regarding the properties of the virus and the experimental procedure, are met. Second, we showed that the accuracy of parameter estimates of influenza A virus infection kinetics and estimates of NAI efficacy depend on the magnitude of the influenza A virus release rate when obtained with a simple MM (with an implicit representation of virus release). MMs are a useful tool that allow for the exploration of known unknowns.

When we investigated the DIP counting assay, we were faced with the first unknowns: the causes behind the curvature in the assay data. In seeking to explain this, we were able to enumerate several causes and organize a set of criteria for the proper application of the assay such that curvature is no longer observed. To demonstrate the causes of curvature, the co-infection window length of a STV-infected cell and the fraction of progeny STV produced from a co-infected cell were manipulated in a straightforward way in the MM. Accomplishing the same manipulations experimentally would be technically challenging. One could imagine that modulation of NA expression to shorten/length the co-infection window of a STV-infected cell might be feasible. However, modulating the fraction of STV produced from a co-infected cell would require knowledge of the mechanism by which DIPs gain an advantage over STVs during viral assembly and packaging. Such interference mechanism has not been elucidated, and could not be experimentally manipulated. MMs make it possible to approximate and achieve this.

When we investigated the simple MM, we confronted the next unknown: the influenza 
A virus release rate. To overcome this, we explored many values of the release rate in order to make comparisons to the simple MM. We found that the simple MM accurately estimates infection parameters but not the NAI efficacy, if virus release is rapid; and vice versa, if virus release is slow. Though it is difficult to measure the virus release rate experimentally, our MM predicts that differences in suppression of influenza A virus strains when different antiviral therapies are applied, might indicate a lower bound on the release rate. Herein, MMs allowed us to pose such questions and highlight the importance of determining the influenza A virus release rate.

In summary, we have developed MMs to simulate the kinetics of co-infection with DIPs and to simulate the explicit release of bound virus from infectious cells. The MMs suggest that there are limitations to each of the quantitative methods that we investigated. These findings will allow others to obtain a nuanced interpretation, and improve the accuracy of, results obtained from such methods. 


\section{Appendix A}

\section{The LIB model}

\section{A.1 Biological basis of key co-infection parameters of in- fluenza A virus}

By simulating a variety of in vitro infection experiments, we determined that influenza A virus infections satisfy the criteria such that the B\&C assay will accurately quantify influenza A DIPs when it is properly performed. In the case of influenza A virus, the length of the co-infection window results from either of two mechanisms which block co-infection from happening: superinfection exclusion [43], and/or the shutdown of viral replication [67]. In superinfection exclusion, the appearance of viral neuraminidase (NA) on the cell surface is responsible for the cleavage of sialic acid receptors, preventing the entry of any more particles midway through the viral replication cycle. With the shutdown of viral replication, the M1 viral protein accumulates in the infected cell's nucleus, inhibiting nuclear import of viral RNA, while facilitating its export out of the nucleus to the cell membrane for assembly and packaging. Consequently, any particles which entered during or after the shutdown would be prevented from replicating their vRNA. The co-infection window is likely a combination of particle entry blockage and the halt in replication. We estimated the co-infection window length to be $3.5 \mathrm{~h}$, which we find is consistent with the timing of NA (blocking particle entry) and M1 (replication shutdown). It has been shown that viral neuraminidase begins appearing on the apical surface of STV-infected cells at $1 \mathrm{~h}-3 \mathrm{~h}$, reaching a maximum at $4 \mathrm{~h}-7 \mathrm{~h}$ postinfection $[12,58]$, while the M1 protein is detectable at $3 \mathrm{~h}$ post-infection [67]. It is not

necessary in the LIB model to distinguish between either mechanism as the cause of the co-infection window in order to faithfully reproduce viral kinetics in the presence of DIPs. 
Our other key co-infection parameter, the fraction of STV progeny produced by a cell co-infected with influenza A STV and DIP, corresponds to the strength of the interference by DIPs. It is believed that DI vRNA has a length advantage over full-length vRNAs, allowing DIPs to competitively inhibit STV replication, but the exact mechanism is unknown [66]. According to one hypothesis generated by a mathematical model [34], DI vRNAs sequester nucleoprotein (NP) to the detriment of full-length vRNAs, since $\mathrm{NP}$ is an essential viral protein required for RNA stabilization [104, 105]. Using this mathematical model, Heldt et al. suggest that such a mechanism of interference would result in the almost exclusive production of progeny DIPs from a co-infected cell [35]. Herein, based on analysis of experimental data by the LIB model, we estimate that cells co-infected by influenza A STV and DIPs do not produce more than 1 PFU for every $10^{4}$ progeny DIPs, consistent with both Heldt et al.'s finding, and the general belief that co-infected cells nearly exclusively produce DIPs. In estimating the fraction of STV progeny produced by STV and DIP co-infected cells, we made another interesting finding. Using the LIB model, we determined that the DIP content in our 2009 pandemic H1N1 influenza A STV stock was approximately 2 DIP/PFU. Ordinarily, such an undesirably high DIP concentration in a STV stock would be passaged at low MOI to lower the DIP content [26]. However, our STV stock was made by reverse genetics, and passaging had to be avoided so as not to introduce mutations. When a drop in the peak value of STV is observed (due to DIPs) between multiple-cycle and single-cycle infections, in our experience, we have found a posteriori that the virus was made by reverse genetics. For example, peak drops of at least three orders of magnitude were observed in [39, 81, 98] for each virus strain generated by reverse genetics.

\section{A.2 Alternative explanation for the reversal of DIP-mediated interference}

The LIB model assumes that the rate of virion (DIP or STV) production by co-infected cells, and the fraction of that progeny consisting of STV rather than DIPs, is independent of the number of STVs and/or DIPs which co-infected the cell. In other words, the LIB model assumes that a cell infected by one STV and two DIPs will produce virions at the same rate, and the same fraction of its progeny will be STV, as a cell infected by five STV and one DIP.

Challenging this notion, Akkina et al. in 1984, suggest that the fraction of STV vs DIP progeny produced by co-infected cells is dictated by the number of infecting particles, i.e., has a dependence on the ratio of infecting PFU to DIP [1]. Specifically, 


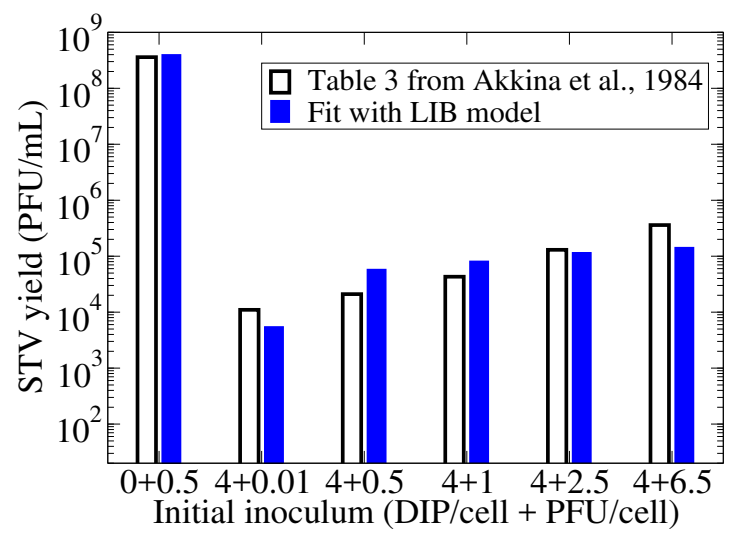

Figure A.1: Evidence by Akkina et al. for a reversal of DIPs' interfering, suppressive effect. STV yield at $24 \mathrm{hpi}$ for infections initiated with the STV and DIP MOIs indicated ( $x$-axis). A reversal of the STV yield suppression by DIPs appears to occur when a cell is super-infected with additional STV, as per the original dataset by Akkina et al. (white bars, [1]). Using the LIB model (blue bars, see Methods in Section A.5), which assumes that co-infected cells will produce the same amount of DIPs and STV, irrespective of the number of STVs or DIPs which infected them, it is possible to reproduce the data by Akkina et al. without the need to assume a reversal of DIP interference.

Akkina et al. propose that the fraction of DIPs produced by co-infected cells is determined by the ratio of full-length, STV vRNAs to DI vRNAs which infect a cell. The idea is that if a cell receives an overwhelming number of STV vRNA copies, this will allow STV vRNA replication to partially overcome the replication advantage held by the shorter-length DI vRNAs. Consequently, according to Akkina et al., further infection of DIP+STV co-infected cells with additional STV should lead to the reversal of the interfering, suppressive effect of DIPs on the STV yield.

Figure A.1 (white bars) presents the data which led Akkina et al. to conclude that DIP-mediated interference is reversible [1]. To summarize their data, a drop in STV yield was observed between the DIP-free control (0 DIP/cell $+0.5 \mathrm{PFU} /$ cell $)$ and an infection resulting from inoculation with STV and DIPs (4 DIP/cell $+0.01 \mathrm{PFU} /$ cell). This demonstrates the suppression of STV yields, typical of DIP interference. However, as the STV MOI was increased while keeping the DIP MOI fixed, Akkina et al. claim to observe increasing STV yields. From this, Akkina et al. concluded that increasing the inoculum STV MOI was responsible for reversing the suppressive effect of DIPs and that a greater fraction of STV progeny is produced by co-infected cells when super-infected by STV. 
While the data presented in figure A.1 are consistent with this hypothesis, they are not conclusive. Figure A.1 also presents the results of an experiment simulated with the LIB model (blue bars) demonstrating that, without having to assume that the fraction of progeny made by co-infected cells is influenced by the STV or DIP MOI, it is possible to account for the trend in these data, even under the assumption that co-infected cells strictly produce DIPs. Our alternative explanation of these data is based on the fact that some of the lower STV MOI inocula used by Akkina et al. leave a pool of uninfected cells which are then available to become STV-infected, producing higher STV yields when the inoculum STV MOI is increased. The Poisson distribution predicts that an inoculum containing $4 \mathrm{DIP} /$ cell will infect $98 \%$ of cells, leaving $2 \%$ of cells free to be infected by any additional STV included in the inoculum. In a typical in vitro infection in 6 -well plates where monolayers contain $\sim 10^{6}$ cells, this would mean $\sim 2 \times 10^{4}$ cells will remain susceptible to STV. Thus, as the STV MOI in the inoculum is increased, so is the fraction of these cells infected by STV alone, manifesting as the increase in STV yield observed by Akkina et al. Therefore, the DIP portion of the initial inoculum should contain at least $14 \mathrm{DIP} /$ cell, instead of $4 \mathrm{DIP} /$ cell, to ensure that all cells are DIP-infected to avoid this effect.

While we can provide an alternative explanation to account for the effect that Akkina et al. claim to have observed, we also question whether these data even show such an effect at all, i.e., whether the reversal is statistically significant or an irreproducible, statistically significant coincidence. This is made more difficult to ascertain since uncertainties (error bars) were not reported by Akkina et al. in [1]. Some infections were initiated with an inoculum containing a low STV MOI (0.01 PFU/cell, 0.5 PFU/cell, $1 \mathrm{PFU} /$ cell), resulting in multiple cycles of infection. As such, the STV yield observed is a function of not just the composition of the initial inoculum, but also of the progeny resulting from the initial infection. To avoid confounding effects from multiple cycles of infection, all inocula used should have been performed at high MOI (i.e., > 4 PFU/cell) to ensure the effects observed are only due to the inocula. Furthermore, replicate experiments should be performed to determine experimental uncertainty and ascertain the statistical significance of any observed change in yield.

Therefore, the experiment performed by Akkina et al. [1] does not demonstrate, in a definitive manner, the reversal of DIP interference in co-infected cells when these cells are super-infected by additional STV. However, a modified version of their experiment, wherein no inoculum contains less than $14 \mathrm{DIP} /$ cell and no less than $4 \mathrm{PFU} /$ cell, should be sufficient to do so. As more and more STV is added to the inoculum containing $14 \mathrm{DIP} /$ cell, the STV progeny, if any, would be that from co-infected cells only, and their 
dependence on the number of infecting particles could be assessed, without confounding STV production from a small pool of STV-only infected cells. Such an experiment should be done using multiple replicates to adequately capture experimental variability and establish the statistical significance of any observed effect. It is interesting to note that Heldt et al. have explored the effect of the number of infecting particles on the progeny produced using a mathematical model of a single cell with a detailed representation of intracellular viral replication. The authors found that a co-infected cell almost exclusively produced DIPs even when there was an increasing number of infecting STV [35]. With the same single cell model [60], Laske et al. matched the results by Akkina et al., however, the reversal of DIP-mediated interference could only recover $1 \%$ of the STV yield. Since the LIB model which considers a population of cells, and the model of a single cell by $[35,60]$, can both reproduce these data, this highlights the fact that these data alone are not sufficient to show that reversal of DIP-mediated interference occurs.

\section{A.3 Sensitivity of the $\mathrm{B} \& \mathrm{C}$ assay to samples containing very high or very low DIP MOIs}

In figure A.2, the B\&C assay is simulated for infections inoculated with the DIP-free STV stock at $4 \mathrm{PFU} /$ cell plus a series of dilutions of example samples containing either $8,16,18$, or $20 \mathrm{DIP} /$ cell when undiluted. The assay curve for the example samples containing greater than $16 \mathrm{DIP} /$ cell plateaus at high DIP doses and no longer follows a linear trend. The plateau is due to the total suppression of infection even when the sample is diluted such that, any further reduction in yield by additional DIPs beyond $16 \mathrm{DIP} /$ cell is masked by the STV leftover post-rinsing.

At first glance, it appears that the $\mathrm{B} \& \mathrm{C}$ assay is only sensitive to a sample containing up to $16 \mathrm{DIP} /$ cell. However, the sensitivity is determined by the peak value of STV in the infection initiated solely by the DIP-free STV stock (stock-only infection). If the STV yield from the DIP-free infection (at DIP dose of zero) is higher, a greater reduction in yield can be observed, making the assay more sensitive to samples with even higher DIP MOI than $16 \mathrm{DIP} /$ cell. The stock-only infection STV peak is ultimately responsible for the sensitivity of the assay, and depends on the virus-cell kinetics (e.g., virus production rate), or the STV stock purity (i.e., whether the stock is contaminated by DIPs). We can say that due to a STV peak of $10^{9} \mathrm{PFU} / \mathrm{mL}$ in our simulated stock-only infection, the $\mathrm{B} \& \mathrm{C}$ assay is suitable for counting samples containing up to $16 \mathrm{DIP} /$ cell. When performing the B\&C assay with an unknown sample, if a plateau at high DIP dose (low or no dilution of the sample) is observed and other causes of curvature have been ruled 


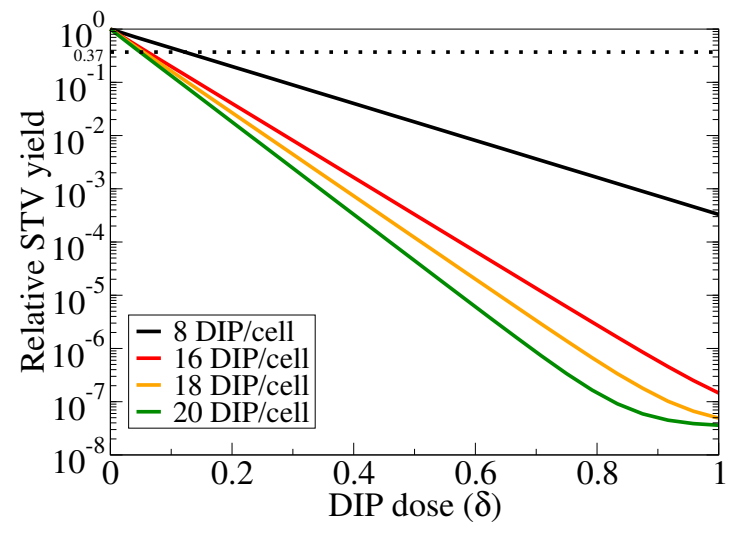

Figure A.2: Impact of the DIP MOI of the sample on the B\&C assay. Simulated $\mathrm{B} \& \mathrm{C}$ assay using the LIB model for cells infected with an inoculum of DIP-free influenza A STV stock at $4 \mathrm{PFU} /$ cell and varying dilutions of an example sample containing either $8,16,18,20 \mathrm{DIP} /$ cell when undiluted.

out (as summarized in Section A.4), a subsequent B\&C assay could be repeated with a dilution of the sample at a lower DIP MOI, i.e., in the range where it remains linear.

For samples with very low DIP MOI, the sensitivity of the B\&C assay is limited by the experimental variability. Since variability is typically half an order of magnitude, i.e., $\pm 0.5 \log _{10}\left(\mathrm{TCID}_{50}\right)$ or $\pm 0.5 \log _{10}(\mathrm{PFU})$, we would expect that a $\mathrm{B} \& \mathrm{C}$ assay curve would be indistinguishable from a totally horizontal curve (i.e., insensitive B\&C assay) when the relative yield from the undiluted sample (at a DIP dose of 1 ) is about 0.5 . This corresponds to an undiluted sample which contains $0.7 \mathrm{DIP} /$ cell (i.e., $-\log (0.5)$ ). Thus, the B\&C assay is sensitive to samples containing DIP MOIs as low as $0.7 \mathrm{DIP} /$ cell and as high as $16 \mathrm{DIP} /$ cell, depending on the stock-only infection STV peak.

\section{A.4 Classification of types and causes of curvature in the $\mathrm{B} \& \mathrm{C}$ assay}

In figure A.3, we summarize the two ways in which non-exponential curvature can manifest in the B\&C assay: at low DIP doses (high dilution of sample) or at high DIP doses (low dilution of sample). We have already shown in figures 3.10 and 3.6 that curvature at low DIP doses is either due to inoculating with a low MOI STV stock (less than $4 \mathrm{PFU} /$ cell), or due to a very long co-infection window, respectively. Likewise, we have shown in figures A.2 and 3.8 that curvature at high DIP dose is either due to a sample containing a very high DIP MOI (greater than $16 \mathrm{PFU} /$ cell, given $10^{9} \mathrm{PFU} / \mathrm{mL}$ 


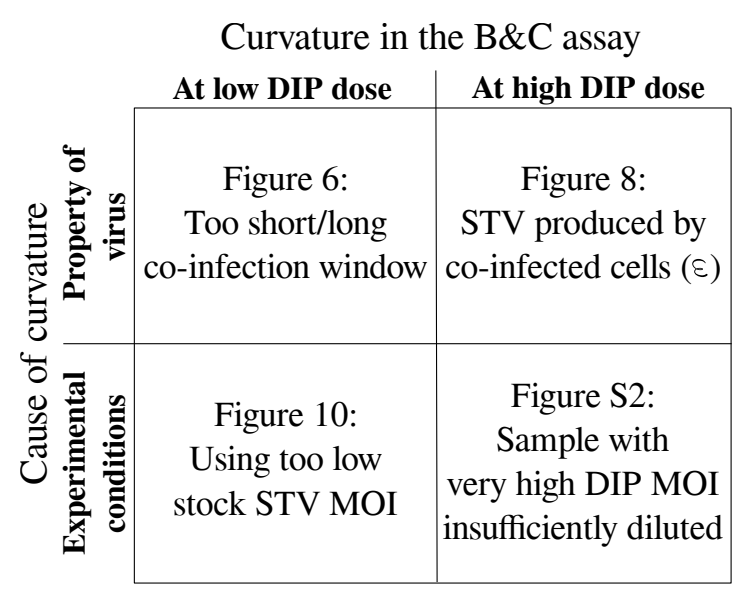

Figure A.3: Classifying types and causes of non-exponential curvature in the $\mathbf{B} \& \mathbf{C}$ assay. Curvature at low DIP dose occurs either when the co-infection window is too short/long, or when the stock STV MOI is too low. Curvature at high DIP dose occurs either when there is too much STV per DIP produced by co-infected cells, or when the DIP MOI of the sample is too high. The curvature in the B\&C assay is either caused by experimental conditions, or stems from an inherent property of the virus.

STV peak in the stock-only infection), or due to a large amount of STV progeny being produced by co-infected cells (greater than 1 PFU per 1,000 DIPs), respectively.

In other words, these curvatures can be caused by either a property of the virus (i.e., co-infection window, STV progeny made by co-infected cells) or by the experimental conditions (i.e., STV stock MOI, sample DIP MOI). Of the two causes, it is relatively easy to adjust experimental conditions such as STV and DIP MOIs. Hence, if we believe that the co-infection window and the fraction of STV progeny from co-infected cells are likely not culprits of the B\&C assay's non-linear curvature, which is supported by figure 3.7 and 3.9, appropriate experimental design should ensure the DIP MOI in an influenza A virus sample can be accurately determined using the $\mathrm{B} \& \mathrm{C}$ assay. Practically, this means that one should perform the B\&C assay using a high STV MOI with the unknown DIP sample, and subsequently assess whether the resulting assay curve appears non-linear on a logarithmic-linear plot. If so, the unknown DIP MOI might be too high, and we suggest diluting the DIP sample and repeating the B\&C assay until linearity in the assay curve is achieved. 


\section{A.5 Methods}

\section{A.5.1 The multiplicity of infection (MOI) determines the fraction of infected cells}

It is well-known that the multiplicity of infection (i.e., the ratio of STV in the inoculum to the number of susceptible cells receiving the inoculum) determines the proportion of cells which receive a STV and get infected. Since the process of randomly tossing particles onto an array of cells is exactly described as a Poisson process, we can use the Poisson distribution to mathematically compute the fraction of cells which receive an exact number $\left(N_{V}\right)$ of STV:

$$
\text { Fraction of cells receiving } N_{V} \mathrm{STV}=\frac{\mathrm{MOI}^{N_{V}} \exp (-\mathrm{MOI})}{N_{V} !}
$$

To compute the fraction of cells that are STV-infected, we must determine the fraction of cells which received one or more STV, which is equivalent to the total proportion of cells excluding those which did not receive any $\operatorname{STV}\left(N_{V}=0\right)$ :

$$
\text { Fraction of STV-infected cells }=1-\frac{\mathrm{MOI}^{0} \exp (-\mathrm{MOI})}{0 !}=1-\exp (-\mathrm{MOI})
$$

In mixed infections, when the inoculum contains both STV and DIPs that are distributed randomly and independently onto cells, the fraction of cells that are STV and DIP co-infected is given by

Fraction of STV+DIP co-infected cells $=[1-\exp (-\mathrm{MOI})][1-\exp (-\mathrm{DIP}$ MOI $)]$

And the remaining fraction of cells in a mixed infection are given by

$$
\begin{aligned}
\text { Fraction of STV-only infected cells } & =[1-\exp (-\mathrm{MOI})] \exp (-\mathrm{DIP} \text { MOI }) \\
\text { Fraction of DIP-only infected cells } & =\exp (-\mathrm{MOI})[1-\exp (-\mathrm{DIP} \text { MOI })] \\
\text { Fraction of uninfected cells } & =\exp (-\mathrm{MOI}) \exp (-\mathrm{DIP} \text { MOI })
\end{aligned}
$$

\section{A.5.2 Mathematical model}

The course of in vitro influenza A virus infections in the presence and absence of DIPs was simulated using a mathematical model. The model is based on a simpler one used in previous work wherein it was validated against experimental data for in vitro influenza A virus infections in the absence of DIPs $[81,85]$. Since interferon does not have a 
significant effect on influenza A virus infections in MDCK cells [96], the LIB model does not include a cellular interferon response. To account for the effect of DIPs on infection kinetics, it was modified based, in part, on an earlier model by [55].

$$
\begin{aligned}
\frac{\mathrm{d} T}{\mathrm{~d} t} & =-\beta T(V+D) & \frac{\mathrm{d} A}{\mathrm{~d} t} & =\beta T D-\beta A V \\
\frac{\mathrm{d} E_{1}}{\mathrm{~d} t} & =\beta T V-\frac{n_{\mathrm{E}}}{\tau_{\mathrm{E}}} E_{1}-z_{1} \beta E_{1} D & \frac{\mathrm{dCE} E_{1}}{\mathrm{~d} t} & =\beta A V-\frac{n_{\mathrm{E}}}{\tau_{\mathrm{E}}} \mathrm{CE}_{1}+z_{1} \beta E_{1} D \\
\frac{\mathrm{d} E_{i=2, \ldots, n_{E}}}{\mathrm{~d} t} & =\frac{n_{\mathrm{E}}}{\tau_{\mathrm{E}}} E_{i-1}-\frac{n_{\mathrm{E}}}{\tau_{\mathrm{E}}} E_{i}-z_{i} \beta E_{i} D & \frac{\mathrm{dCE}_{i=2, \ldots, n_{E}}}{\mathrm{~d} t} & =\frac{n_{\mathrm{E}}}{\tau_{\mathrm{E}}} \mathrm{CE}_{i-1}-\frac{n_{\mathrm{E}}}{\tau_{\mathrm{E}}} \mathrm{CE}_{i}+z_{i} \beta E_{i} D \\
\frac{\mathrm{d} I_{1}}{\mathrm{~d} t} & =\frac{n_{\mathrm{E}}}{\tau_{\mathrm{E}}} E_{n_{\mathrm{E}}}-\frac{n_{\mathrm{I}}}{\tau_{\mathrm{I}}} I_{1} & \frac{\mathrm{dCI}}{\mathrm{d} t} & =\frac{n_{\mathrm{E}}}{\tau_{\mathrm{E}}} \mathrm{CE}_{n_{\mathrm{E}}}-\frac{n_{\mathrm{I}}}{\tau_{\mathrm{I}}} \mathrm{CI}_{1} \\
\frac{\mathrm{d} I_{j=2, \ldots, n_{I}}}{\mathrm{~d} t} & =\frac{n_{\mathrm{I}}}{\tau_{\mathrm{I}}} I_{j-1}-\frac{n_{\mathrm{I}}}{\tau_{\mathrm{I}}} I_{j} & \frac{\mathrm{dCI}_{j=2, \ldots, n_{I}}}{\mathrm{~d} t} & =\frac{n_{\mathrm{I}}}{\tau_{\mathrm{I}}} \mathrm{CI}_{j-1}-\frac{n_{\mathrm{I}}}{\tau_{\mathrm{I}}} \mathrm{CI}_{j} \\
\frac{\mathrm{d} V}{\mathrm{~d} t} & =p \sum_{j=1}^{n_{\mathrm{I}}} I_{j}+\varepsilon p \sum_{j=1}^{n_{\mathrm{I}}} \mathrm{CI}_{j}-c V & \frac{\mathrm{d} D}{\mathrm{~d} t} & =(1-\varepsilon) p \sum_{j=1}^{n_{\mathrm{I}}} \mathrm{CI}_{j}-c D
\end{aligned}
$$

Equations on the left-hand side correspond to the standard infection model in which uninfected target cells $(T)$ are infected by $\operatorname{STV}(V)$ or DIPs $(D)$, at infection rate $\beta$. The STV-infected eclipse cells $(E)$ remain infected and non-producing for a time called the eclipse length $\tau_{E}$, after which they become infectious cells $(I)$, and produce STV over a length of time called the infectious phase $\tau_{I}$. The STV progeny are produced and released at rate $p$, and lose infectivity at rate $c$. The transition times between cell states (eclipse to infectious, infectious to dead) follow an Erlang distribution whose mean is given by $\tau$, and standard deviation by $\sigma=\frac{\tau}{\sqrt{n}}$. The parameter $n_{E}\left(n_{I}\right)$ is the number of compartments the eclipse (infectious) phase is divided into, where the compartments are indexed by $i(j)$. The parameter $z_{i}=1$ for $i=1:\left(\frac{\tau_{C}}{\tau_{E}}\right) n_{E}$ opens the co-infection window $\left(\tau_{C}\right)$ to allow transitions from the standard to co-infected branch, and is zero otherwise.

The co-infected branch (on the right-hand side) describes co-infection of the DIPinfected arrested cells $(A)$. Cells become co-infected eclipse cells $(\mathrm{CE})$ and co-infected infectious cells (CI) with an eclipse and infectious phase of length $\tau_{E}$ and $\tau_{I}$, respectively. Co-infected infectious cells release a fraction $(1-\varepsilon)$ of progeny DIPs and a fraction $\varepsilon$ of progeny STV with production rate $p$. The DIPs also lose infectivity at rate $c$.

We use best fit parameter estimates from [81] where the infection kinetics of the 2009 pandemic influenza A/Québec/144147/09 (H1N1) virus strain, as published in [85], was re-analyzed (figure 3.2). Table A.1 lists our base parameter values. 
Table A.1: Base parameter values and $\mathbf{9 5 \%}$ confidence intervals for infection with an influenza A (H1N1) pandemic strain [81].

\begin{tabular}{lc} 
Parameter, symbol & Base value $[95 \% \mathrm{CI}]$ \\
\hline Production rate, $p\left((\mathrm{PFU} / \mathrm{mL}) \cdot \mathrm{h}^{-1}\right)$ & $3.27 \times 10^{8}[1.66-7.50]$ \\
Fraction of progeny $\mathrm{STV}$ produced by co-infected cells, $\varepsilon$ & 0 \\
Infection rate, $\beta\left((\mathrm{PFU} / \mathrm{mL})^{-1} \cdot \mathrm{h}^{-1}\right)$ & $2.44 \times 10^{-7}[1.17-7.66]$ \\
Clearance rate, $c\left(\mathrm{~h}^{-1}\right)$ & $0.14[0.12-0.18]$ \\
Co-infection window length, $\tau_{C}(\mathrm{~h})$ & 3 \\
Eclipse length, $\tau_{E}(\mathrm{~h})$ & $6.58[6.04-7.73]$ \\
Infectious length, $\tau_{I}(\mathrm{~h})$ & $46.8[37.4-54.4]$ \\
Number of eclipse compartments, $n_{E}$ & 60 \\
Number of infectious compartments, $n_{I}$ & 60 \\
Rinsing factor, $f_{\text {resid }}$ & $8 \times 10^{-5}$ \\
Adsorption time, $t_{\text {rinse }}(\mathrm{h})$ & 1
\end{tabular}

When simulating an infection where the inoculum is incubated for one hour, then rinsed and replaced with fresh virus-free medium, when we assert that the sample contains $0.04 \mathrm{PFU} /$ cell we mean that in simulating this infection with our kinetic mathematical model, $4 \%$ of cells will become infected by the inoculum under these conditions (1h incubation then rinse). As such, using 100x that same inoculum will correspond to an MOI of $4 \mathrm{PFU} /$ cell. Just like in actual experiments, quantification of infectivity in our simulated inoculum is defined relative to both the specific cell type in which the infection proceeds (simulating a different cell type corresponds to changing cell-specific mathematical model parameters such as $p, \beta, \tau_{E}, \tau_{I}$, etc...), and also relative to the chosen infection methodology (rinsing after $1 \mathrm{~h}$ incubation vs no rinse vs longer incubation period, etc...).

\section{A.5.3 Simulating infections}

Infections are simulated by applying an initial inoculum $\left(V_{0}\right)$ to a population of fully susceptible cells $\left(T_{0}=1\right)$. The inoculum's STV concentration is characterized by the desired multiplicity of infection (MOI) and determined in the following way

$$
V_{0}=\frac{c}{\beta} \frac{\mathrm{MOI}}{1-\exp \left(-c t_{\text {rinse }}\right)}
$$

If the initial inoculum contains DIPs, the DIP concentration $\left(D_{0}\right)$ is determined in an analogous way. The initial inoculum is allowed to adsorb for a time $t_{\text {rinse }}$, before a rinse is simulated by reducing the particle concentrations by a factor $f_{\text {resid }}$. 


\section{$\mathrm{B} \& \mathrm{C}$ reduction of $\mathrm{STV}$ yield assay}

In the $\mathrm{B} \& \mathrm{C}$ assay [6], we are interested in the relative STV yield between an infection initiated with just the STV stock (stock-only infection), and infections initiated with both the STV stock plus dilutions of the DIP sample (stock-plus-sample infection). The stock-only infection is initiated with a STV MOI of $4 \mathrm{PFU} /$ cell. The stock-plus-sample infections are initiated with the $4 \mathrm{PFU} /$ cell STV stock, in addition to $\delta(8 \mathrm{DIP} /$ cell $)$ of the sample, where $0 \leq \delta \leq 1$ denotes the DIP dose. The B\&C assay is simulated using base parameters unless otherwise noted. The 20 hpi STV yield from the stock-plus-sample infections are measured and normalized to that of the stock-only infection, in order to form the assay curve of relative STV yield as a function of DIP dose.

To estimate the percent error in the estimate of DIP MOI from B\&C, the assay was performed for combinations of stock and sample at $10^{-3} \mathrm{PFU} /$ cell- $10^{1} \mathrm{PFU} /$ cell and $10^{-3} \mathrm{DIP} /$ cell-10 $10^{1} \mathrm{DIP} /$ cell, respectively. The sample DIP MOI was estimated from the reciprocal of the DIP dose which coincided with $37 \%$ relative STV yield, as in [65]. In some cases where the assay curve remained above, and did not cross the $37 \%$ relative STV yield, the DIP dose corresponding to $37 \%$ relative yield was linearly extrapolated. Explicitly, the percent errors were calculated with

$$
\text { Percent error }=\left(\frac{\text { Estimated sample DIP MOI }}{\text { Actual sample DIP MOI }}-1\right)
$$

\section{Nayak's time of (DIP) addition}

To simulate Nayak's time of addition curve in [75], we are also interested in relative STV yield between a stock-only infection and stock-plus-sample infections, where the DIP-containing sample is applied at times subsequent to application of the STV stock. In the stock-plus-sample infections, the STV stock is adsorbed for $0.5 \mathrm{~h}$, followed by rinsing. The time of STV stock application is defined as $0 \mathrm{~h}$. After the STV stock application at $0 \mathrm{~h}$, the sample (containing both DIPs and STV) is subsequently applied at various addition times, adsorbed for $0.5 \mathrm{~h}$, rinsed, and the infection proceeds. One infection received the DIP sample $1 \mathrm{~h}$ before STV stock inoculation, corresponding to a $-1 \mathrm{~h}$ time of addition. The STV yield is measured at $14 \mathrm{~h}$ after STV stock application, and normalized to the STV yield of the stock-only infection.

Using Engauge digitizer, we extracted data on the $14 \mathrm{~h}$ relative STV yield as a function of DIP addition time from [75]. Nayak et al. report the DIP MOI of their sample in units of $\mathrm{PFU} /$ cell, where the authors have assumed that DIPs are approximately 1,000 times less infectious than PFUs. As such, they estimated the sample DIP MOI 
as $4 \mathrm{DIP} /$ cell $(=0.004 \mathrm{PFU} /$ cell $\times 1000 \mathrm{DIP} / \mathrm{PFU})$. Due to the uncertainty in both the concentrations of STV and DIPs, we allowed the LIB model to fit both the STV and DIP MOIs of the stock and sample, in addition to the model parameters. The best fit to these data yields a sum-of-squared residual (SSR) of 0.0032, where the set of best fit parameters is given in Table A.2. Note that the sample's STV MOI was scaled by the stock STV MOI according to $\frac{0.68 \mathrm{PFU} / \text { cell }}{1 \mathrm{PFU} / \text { cell }} \times 0.004 \mathrm{PFU} /$ cell, and was not one of the fitted parameters. Importantly, we assumed that co-infected cells exclusively produce DIPs ( $\varepsilon=0$; base value), however our exploration of $\varepsilon \leq 10^{-3}$ allowed us to obtain fits with equivalent SSRs, with no impact on the extracted co-infection window or eclipse length.

Table A.2: Best fit parameters to Nayak's time of DIP addition data.

\begin{tabular}{lc} 
Parameter, symbol & Fitted value \\
\hline Production rate, $p$ & $1.22 \times 10^{7}(\mathrm{PFU} / \mathrm{mL}) \cdot \mathrm{h}^{-1}$ \\
Fraction of progeny STV produced by co-infected cells, $\varepsilon$ & $0(\mathrm{fixed})$ \\
Infection rate, $\beta$ & $9.83 \times 10^{-2}(\mathrm{PFU} / \mathrm{mL})^{-1} \cdot \mathrm{h}^{-1}$ \\
Clearance rate, $c$ & $0.04 \mathrm{~h}^{-1}$ \\
Co-infection window length, $\tau_{C}$ & $3.5 \mathrm{~h}$ \\
Eclipse length, $\tau_{E}$ & $7.11 \mathrm{~h}$ \\
Infectious length, $\tau_{I}$ & $46.8 \mathrm{~h}($ fixed $)$ \\
Number of eclipse compartments, $n_{E}$ & $60($ fixed $)$ \\
Number of infectious compartments, $n_{I}$ & $60($ fixed $)$ \\
Rinsing factor, $f_{\text {resid }}$ & $8 \times 10^{-5}($ fixed $)$ \\
Adsorption time, $t_{\text {rinse }}$ & $0.5 \mathrm{~h}($ fixed $)$ \\
Stock STV MOI & $0.68 \mathrm{PFU} / \mathrm{cell}$ \\
Sample DIP MOI & $2.30 \mathrm{DIP} / \mathrm{cell}$ \\
Sample STV MOI & 0.0032 \\
Sum-of-squared residuals, SSR & $\mathrm{PFU} / \mathrm{cell}(\mathrm{computed})$ \\
\hline
\end{tabular}

The reliability of the co-infection window estimate was evaluated by assessing the sensitivity of the Nayak et al. time of addition curve to changes in infection kinetics. Figure A.4 shows that the curve is only re-scaled in height, such that the location of the rise remains relatively unchanged when using a DIP-free inoculum of $1 \mathrm{PFU} /$ cell: estimation of the co-infection window is mostly robust, within $\pm 0.5 \mathrm{~h}$. When the same sensitivity analysis is simulated using an inoculum with a higher STV MOI (4 PFU/cell), the estimate can be made even more robust, where the location of the rise is insensitive to most infection parameters, except for the eclipse length. 

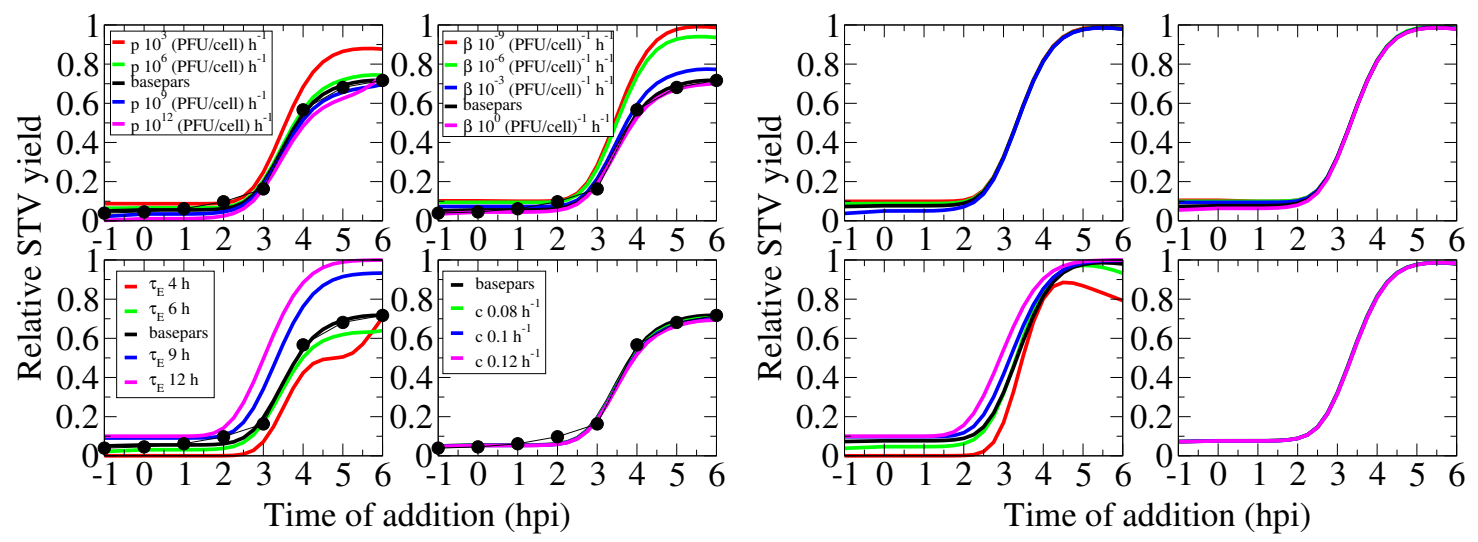

Figure A.4: Evaluating the reliability of the co-infection window estimated from the Nayak et al. assay. Infections were simulated as in figure 3.7, with the best fit parameters as base values (thick black line), for simulated infections with a DIP-free STV inoculum of: (Left) $1 \mathrm{PFU} /$ cell or (Right) $4 \mathrm{PFU} /$ cell. The production rate, infection rate, clearance rate, and eclipse length are varied about their base value (clockwise from top left panel), for each of the two inocula. The original data from [75] are shown as black circles.

\section{STV peak drop between multiple-cycle and single-cycle infections of pan- demic H1N1}

We simulated the STV peak drop between a multiple-cycle (MC) and single-cycle (SC) infection that is caused by the presence of DIPs in the STV stock used to inoculate both infections, specifically in [85]. Experimentally, the MC infection is performed with a low STV MOI such that the chance of co-infection with DIPs is low. When DIP interference is negligible, we expect to observe a STV peak concentration that is characteristic of a DIP-free infection. The DIP-free STV peak in an MC infection is equivalent to that of an SC infection. Hence, instead of simulating the MC infection, we simply simulate a DIP-free SC infection, initiated with a $4 \mathrm{PFU} /$ cell STV MOI and $1 \mathrm{~h}$ adsorption followed by rinsing, to obtain the DIP-free STV peak sampled at $30 \mathrm{hpi}$.

To obtain the STV peak drop, we simulate SC infections initiated with a $4 \mathrm{PFU} /$ cell STV MOI and increasing DIP MOIs from $10^{-2} \mathrm{DIP} /$ cell $-4 \times 10^{1} \mathrm{DIP} /$ cell. The $30 \mathrm{hpi}$ STV peak from these DIP-containing SC infections are normalized to the DIP-free STV peak, which give us the STV peak drop. In these simulations, we used the base parameters in Table A.1. 


\section{Akkina's reversal of DIP-mediated interference}

To simulate the reversal of DIP-mediated interference in [1], infections are initiated with initial inocula containing the STV and DIP MOIs given in figure A.1. The 24 hpi STV yields were sampled.

We took published data from Table 3 of [1] which shows the STV yield, measured at $24 \mathrm{hpi}$, as a function of increasing STV MOI. A best fit to these data yielded an SSR of 0.528 , with best fit parameters given in Table A.3.

Table A.3: Best fit parameters to Akkina's reversal of DIP-mediated interference data.

Parameter, symbol Fitted value

$\begin{array}{lc}\text { Production rate, } p & 4.91 \times 10^{7}(\mathrm{PFU} / \mathrm{mL}) \cdot \mathrm{h}^{-1} \\ \text { Fraction of progeny STV produced by co-infected cells, } \varepsilon & 0 \\ \text { Infection rate, } \beta & 2.43(\mathrm{PFU} / \mathrm{mL})^{-1} \cdot \mathrm{h}^{-1} \\ \text { Clearance rate, } c & 0.1 \mathrm{~h}^{-1} \\ \text { Co-infection window length, } \tau_{C} & 4 \mathrm{~h} \\ \text { Eclipse length, } \tau_{E} & 6.58 \mathrm{~h} \\ \text { Infectious length, } \tau_{I} & 35 \mathrm{~h} \\ \text { Number of eclipse compartments, } n_{E} & 30 \text { (fixed) } \\ \text { Number of infectious compartments, } n_{I} & 30 \text { (fixed) } \\ \text { Rinsing factor, } f_{\text {resid }} & 3.02 \times 10^{-41} \\ \text { Adsorption time, } t_{\text {rinse }} & 1 \mathrm{~h} \text { (fixed) } \\ \text { STV and DIP MOI } & \text { as indicated (fixed) } \\ \text { Sum-of-squared residuals, SSR } & 0.528\end{array}$




\section{Appendix B}

\section{The release $\mathrm{MM}$}

\section{B.1 Determination of critical release rates}

The critical free virus release rate $\left(r_{f}\right)$ is the minimum rate of viral release that causes the free virus titer in the release MM to overlap with the baseline free virus titer (i.e., the MC infection simulated by the simple MM) to a degree which they can be considered indistinguishable from each other. To quantitatively determine $r_{f}$, the distance between the free virus curves was computed using the sum-of-squared residuals per point (SSR/pt), where a smaller SSR/pt indicates a higher degree of similarity between curves. In Figure B.1 (left), the SSR/pt as a function of release rate was computed between the baseline free virus and each free virus curve from the release MM (Figure 4.2A, red vs. black). The value of the SSR/pt which denoted overlapping curves was set to the variance associated with a mock-yield experiment, $\sigma_{\mathrm{MY}}^{2}=(0.072 \mathrm{PFU} / \mathrm{ml})^{2}=5.184 \times 10^{-3}(\mathrm{PFU} / \mathrm{ml})^{2}$ (horizontal dotted line; computed from [85], data not shown). When all infection parameters were at their base value, the critical free virus release rate was $r_{f}=3.72 \mathrm{~h}^{-1}$. 

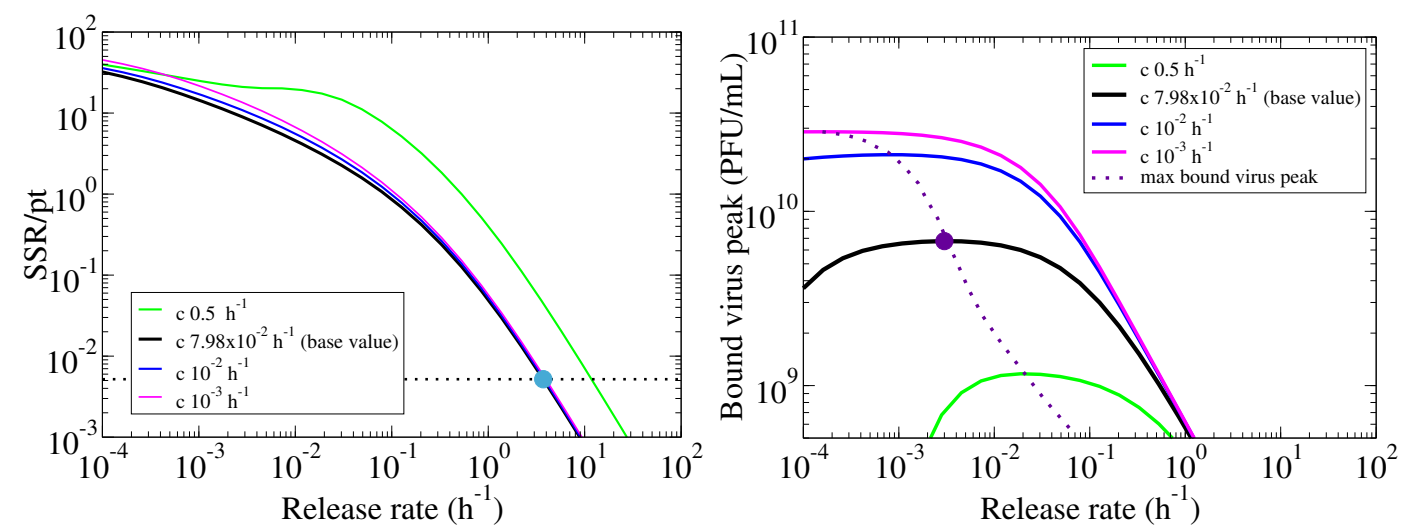

Figure B.1: Determination of critical free and bound virus release rates, $r_{f}$ and $r_{b}$. (Left) The sum-of-squared residuals per point (SSR/pt) is computed between each free virus curve in the release MM, as the release rate is varied, and the simulated MC data in the simple MM (black). The critical free virus release rate, $r_{f}=3.72 \mathrm{~h}^{-1}$ (teal circle), corresponds to the variance of a mock-yield infection (dotted line). We also show the SSR/pt curve for various rates of loss of infectious virion that were explored (various colours). (Right) The peak value of bound virus titer as a function of the release rate is shown (black), where the maximum determines the critical bound virus release rate, $r_{b}=3 \times 10^{-3} \mathrm{~h}^{-1}$ (purple circle). The various coloured lines correspond to various rates of loss of virion infectivity to show that $r_{b}$ (dotted purple) strongly depends on other infection parameters.

To verify whether $r_{f}$ was an absolute value, or if it depended on the infection parameters, the parameters were individually varied away from their base values, and the $\mathrm{SSR} / \mathrm{pt}$ curve was constructed again. In Figure B.1 (left) we illustrate this procedure as the rate of loss of infectious virion, $c$, is varied. Lowering $c$ to $10^{-3} \mathrm{~h}^{-1}$ shifted the $\mathrm{SSR} / \mathrm{pt}$ curve to the left, indicating a lower $r_{f}$. In Figure B.2, we show that $r_{f}$ only varied between $3 \mathrm{~h}^{-1}-5 \mathrm{~h}^{-1}$ for biologically reasonable values of the rate of loss of virion infectivity $\left(c<0.2 \mathrm{~h}^{-1}\right)$. We repeated this procedure for the remaining parameters and found that $r_{f}$ weakly depends on the virus production rate, infection rate, eclipse length and infectious lifespan (Figure B.2). Generally, however, $r_{f}$ remains within the narrow range quoted. 


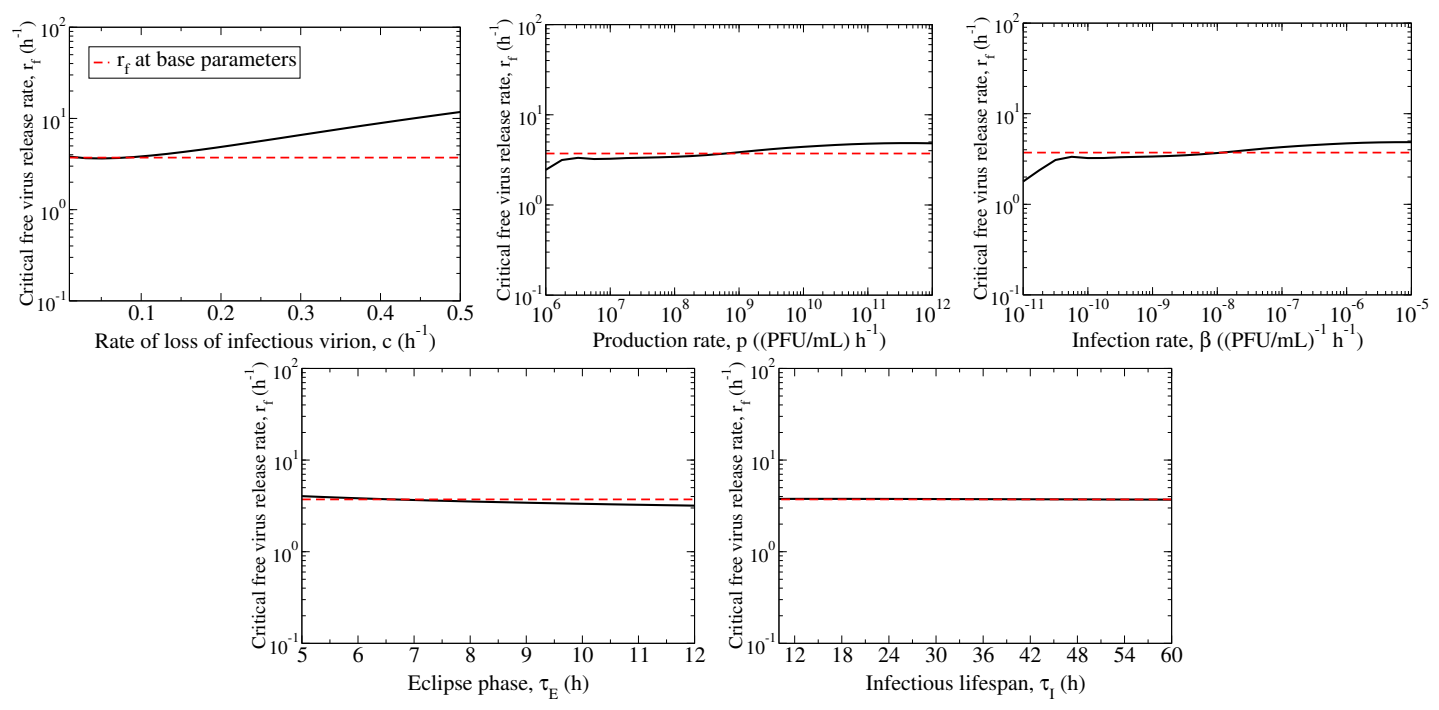

Figure B.2: The critical free virus release rate, $r_{f}$, weakly depends on infection parameters. The critical free virus release rate, $r_{f}$, weakly depends on the rate of loss of virion infectivity, production rate, infection rate, eclipse phase and infectious lifespan. The $r_{f}$ when all parameters are at their base values is indicated with a horizontal dotted line.

To quantitatively determine the critical bound virus release rate, Figure B.1 (right) plots the peak value of bound virus as a function of the release rate. The maximum of the bound virus peak values determines $r_{b}$, indicating whether bound virus loses infectivity faster than it can be released $\left(r<r_{b}\right)$, or vice versa $\left(r>r_{b}\right)$. When all other parameters were at their base values, $r_{b}$ was approximately $3 \times 10^{-3} \mathrm{~h}^{-1}$. Again, parameters were varied from their base values to determine their influence on $r_{b}$. In Figure B.3, $r_{b}$ was dependent on the rate of loss of virion infectivity, production rate, infection rate, and the infectious lifespan. 

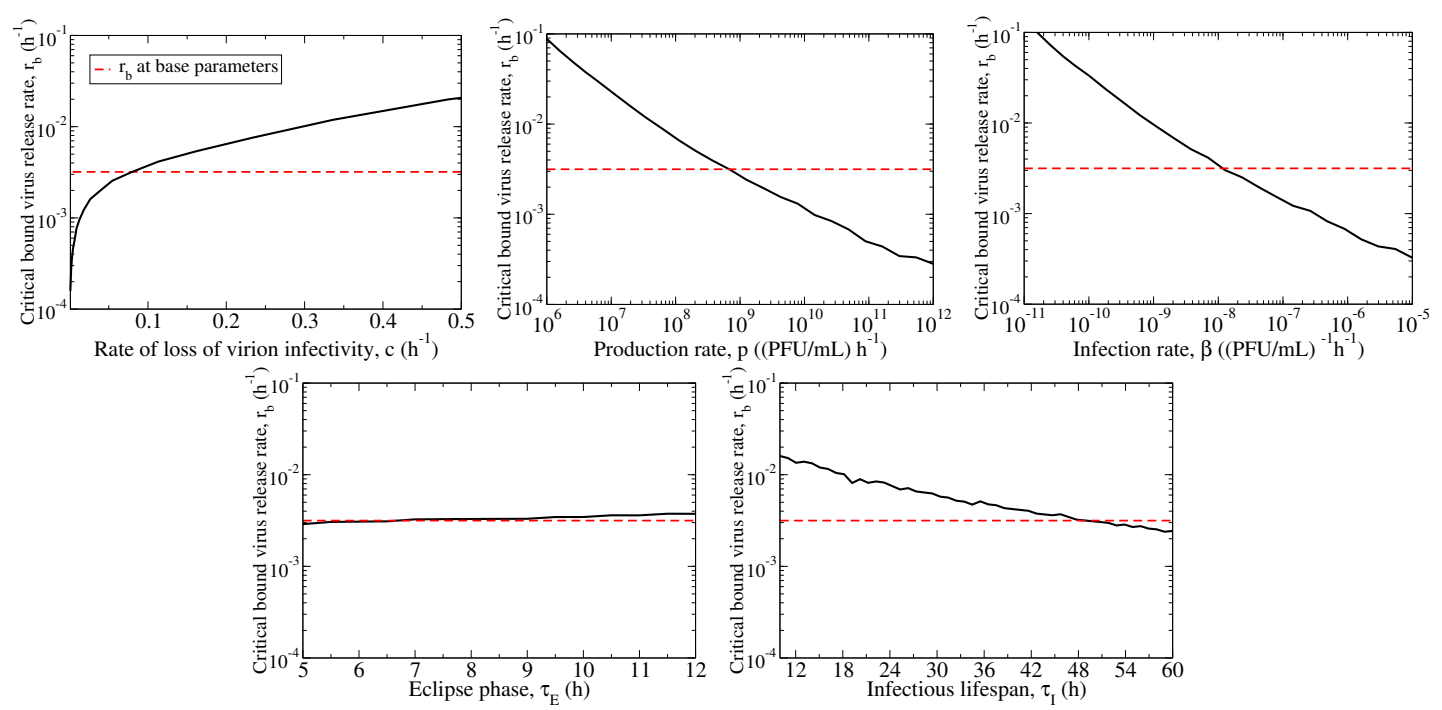

Figure B.3: The critical bound virus release rate, $r_{b}$, depends on infection parameters. The critical bound virus release rate, $r_{b}$, depends on the rate of loss of virion infectivity, production rate, infection rate, eclipse phase, and infectious lifespan. The $r_{b}$ when all parameters are at their base values is indicated with a black circle.

\section{B.2 Infection parameters predicted by the release MM}

In Section 4.2.2, the release MM was fitted to simulated MC, SC, MY data from the simple MM. The simple MM did not significantly misestimate the infection rate or rate of loss of virion infectivity, as shown in Figure B.4. We also examined a derived parameter, the basic reproductive number, $R_{0}$. The $R_{0}$ is defined as the number of secondary infections caused by a single infectious cell in a population of fully susceptible cells, and combines the influence of all the estimated infection parameters except for the eclipse length (Equation (4.8)). Figure B.4 shows that the simple MM's estimate of $R_{0}$ does not significantly differ from the predicted value in the release MM. 

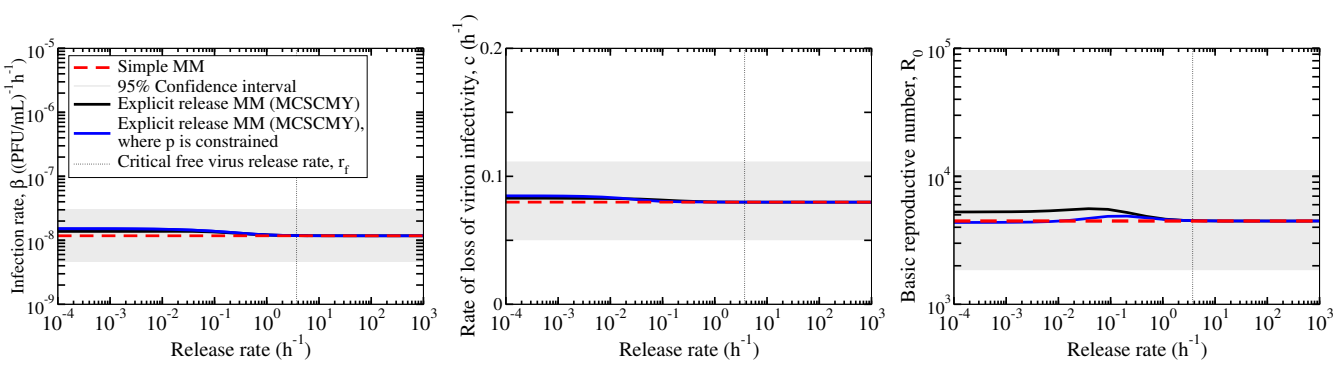

Figure B.4: The simple MM does not significantly misestimate the infection rate, rate of loss of virion infectivity, or the basic reproductive number. As in Figure 4.4, but showing the infection rate, rate of loss of virion infectivity, and the basic reproductive number. 


\section{Bibliography}

[1] Akkina, R. K., Chambers, T. M., and Nayak, D. P. (1984). Mechanism of interference by defective-interfering particles of influenza virus: Differential reduction of intracellular synthesis of specific polymerase proteins. Virus Res., 1(8):687-702.

[2] Baccam, P., Beauchemin, C., Macken, C. A., Hayden, F. G., and Perelson, A. S. (2006). Kinetics of influenza A virus infection in humans. J. Virol., 80(15):7590-7599.

[3] Beauchemin, C. A. A. and Handel, A. (2011). A revew of mathematical models of influenza A infections within a host or cell culture: Lessons learned and challenges ahead. BMC Public Health, 11(Suppl 1):S7.

[4] Beauchemin, C. A. A., McSharry, J. J., Drusano, G. L., Nguyen, J. T., Went, G. T., Ribeiro, R. M., and Perelson, A. S. (2008). Modeling amantadine treatment of influenza A virus in vitro. J. Theor. Biol., 254(2):439-451.

[5] Bedford, T., Riley, S., Barr, I. G., Broor, S., Chadha, M., Cox, N. J., Daniels, R. S., Gunasekaran, C. P., Hurt, A. C., Kelso, A., Klimov, A., Lewis, N. S., Li, X., McCauley, J. W., Odagiri, T., Potdar, V., Rambaut, A., Shu, Y., Skepner, E., Smith, D. J., Suchard, M. A., Tashiro, M., Wang, D., Xu, X., Lemey, P., and Russell, C. A. (2015). Global circulation patterns of seasonal influenza viruses vary with antigenic drift. Nature, 523(7559):217-220.

[6] Bellett, A. J. D. and Cooper, P. D. (1959). Some properties of the transmissible interfering component of vesicular stomatitis virus preparations. J. Gen. Microbiol., 21(3):498-509.

[7] Berry, R. M. and Nowak, M. A. (1994). Defective escape mutants of HIV. J. Theor. Biol., 171(4):387-395.

[8] Boianelli, A., Nguyen, V. K., Ebensen, T., Schulze, K., Wilk, E., Sharma, N., Stegemann-Koniszewski, S., Bruder, D., Toapanta, F. R., Guzmanán, C. A., Meyer- 
Hermann, M., and Hernandez-Vargas, E. A. (2015). Modeling influenza virus infection: A roadmap for influenza research. Viruses, 7(10):5274-304.

[9] Burnham, K. P. and Anderson, D. R. (2002). Model Selection and Multimodel Inference: A Practical Information-Theoretic Approach. Springer-Verlag New York Inc., New York, USA, 2nd edition.

[10] Carter, M. J. and Mahy, B. W. J. (1981). Incomplete avian influenza virus contains a defective non-interfering component. Arch. Virol., 71(1):13-25.

[11] Chan, J., Holmes, A., and Rabadan, R. (2010). Network analysis of global influenza spread. PLoS Comput. Biol., 6(11):e1001005.

[12] Daniels, P. U. and Edwardson, J. (1989). Influenza neuraminidase is delivered directly to the apical surface of MDCK cell monolayers. FEBS Lett., 244(1):57-60.

[13] Davis, A. R., Hiti, A. L., and Nayak, D. P. (1980). Influenza defective interfering viral RNA is formed by internal deletion of genomic RNA. Proc. Natl. Acad. Sci. U.S.A., 77(1):215-219.

[14] Davis, A. R. and Nayak, D. P. (1979). Sequence relationships among defective interfering influenza viral RNAs. Proc. Natl. Acad. Sci. U.S.A., 76(7):3092-3096.

[15] Dimmock, N. J. and Easton, A. J. (2014). Defective interfering influenza virus RNAs: Time to reevaluate their clinical potential as broad-spectrum antivirals? $J$. Virol., 88(10):5217-5227.

[16] Dobrovolny, H. M., Baron, M. J., Gieschke, R., Davies, B. E., Jumbe, N. L., and Beauchemin, C. A. A. (2010). Exploring cell tropism as a possible contributor to influenza infection severity. PLoS ONE, 5(11):e13811.

[17] Dobrovolny, H. M., Gieschke, R., Davies, B. E., Jumbe, N. L., and Beauchemin, C. A. A. (2011). Neuraminidase inhibitors for treatment of human and avian strain influenza: A comparative modeling study. J. Theor. Biol., 269(1):234-244.

[18] Dobrovolny, H. M., Reddy, M. B., Kamal, M. A., Rainer, C. R., and Beauchemin, C. A. A. (2013). Assessing mathematical models of influenza infections using features of the immune response. PLoS ONE, 8(2):e57088.

[19] Duhaut, S. D. and McCauley, J. W. (1996). Defective RNAs inhibit the assembly of influenza virus genome segments in a segment-specific manner. Virology, 216(2):326337. 
[20] Fiore, A. E., Shay, D. K., Broder, K., Iskander, J. K., Uyeki, T. M., Mootrey, G., Bresee, J. S., Cox, N. J., Centres for Disease Control and Prevention (CDC), and Advisory Committee on Immunization Practices (ACIP) (2008). Prevention and control of influenza: recommendations of the Advisory Committee on Immunization Practices (ACIP), 2008. MMWR Morb. Mortal. Wkly. Rep., 57(RR-7):1-60.

[21] Fodor, E., Pritlove, D. C., and Brownlee, G. G. (1994). The influenza virus panhandle is involved in the initiation of transcription. J. Virol., 68(6):4092-4096.

[22] Forkel, D. S. (2016). A model for the intracellular kinetics of infection with the influenza A virus in vitro. Master's thesis, Department of Physics, Ryerson University, Toronto, ON, Canada.

[23] Frank, S. A. (2000). Within-host spatial dynamics of viruses and defective interfering particles. J. Theor. Biol., 206(2):279-290.

[24] Frensing, T. (2015). Defective interfering viruses and their impact on vaccines and viral vectors. Biotechnol. J., 10(5):681-689.

[25] Frensing, T., Heldt, F. S., Pflugmacher, A., Behrendt, I., Jordan, I., Flockerzi, D., Genzel, Y., and Reichl, U. (2013). Continuous influenza virus production in cell culture shows a periodic accumulation of defective interfering particles. PLoS ONE, $8(9): \mathrm{e} 72288$.

[26] Frensing, T., Pflugmacher, A., Bachmann, M., Peschel, B., and Reichl, U. (2014). Impact of defective interfering particles on virus replication and antiviral host response in cell culture-based influenza vaccine production. Appl. Microbiol. Biotechnol, 98(21):8999-9008.

[27] Furuta, Y., Gowen, B. B., Takahashi, K., Shiraki, K., Smee, D. F., and Barnard, D. L. (2013). Favipiravir (T-705), a novel viral RNA polymerase inhibitor. Antiviral Res., 100(2):446-454.

[28] Gambhir, M., Bozio, C., O’Hagan, J. J., Uzicanin, A., Johnson, L. E., Biggerstaff, M., and Swerdlow, D. L. (2015). Infectious disease modeling methods as tools for informing response to novel influenza viruses of unknown pandemic potential. Clin. Infect. Dis., 60(Suppl 1):S11-S19.

[29] Gaymard, A., Briand, N. L., Frobert, E., Lina, B., and Escuret, V. (2016). Functional balance between neuraminidase and haemagglutinin in influenza viruses. Clin. Microbiol. Infect., 22(12):975-983. 
[30] Gubareva, L. V., Nedyalkova, M. S., Novikov, D. V., Murti, K. G., Hoffmann, E., and Hayden, F. G. (2002). A release-competent influenza A virus mutant lacking the coding capacity for the neuraminidase active site. J. Gen. Virol., 83(11):2683-2692.

[31] Handel, A., Akin, V., Pilyugun, S. S., Zarnitsyna, V., and Antia, R. (2014). How sticky should a virus be? The impact of virus binding and release on transmission fitness using influenza as an example. J. R. Soc. Interface, 11(92):20131083.

[32] Handel, A., Longini Jr., I. M., and Antia, R. (2007). Neuraminidase inhibitor resistance in influenza: Assessing the danger of its generation and spread. PLoS Comput. Biol., 3(12):e240.

[33] Handel, A., Longini Jr., I. M., and Antia, R. (2010). Towards a quantitative understanding of the within-host dynamics of influenza A infections. J. R. Soc. Interface, $7(42): 35-47$.

[34] Heldt, F. S., Frensing, T., and Reichl, U. (2012). Modeling the intracellular dynamics of influenza virus replication to understand the control of viral RNA synthesis. $J$. Virol., 86(15):7806-7817.

[35] Heldt, S. (2015). Mathematical models of influenza A virus infection: From intracellular replication to virus growth in cell populations. $\mathrm{PhD}$ thesis, Otto-von-Guericke Universität, Magdeburg, Germany.

[36] Ho, D. D., Neumann, A. U., Perelson, A. S., Chen, W., Leonard, J. M., and Markowitz, M. (1995). Rapid turnover of plasma virions and CD4 lymphocytes in HIV-1 infection. Nature, 373(6510):123-126.

[37] Holder, B. P. and Beauchemin, C. A. A. (2011). Exploring the effect of biological delays in kinetic models of influenza within a host or cell culture. BMC Public Health, 11(Suppl 1):S10.

[38] Holder, B. P., Liao, L. E., Simon, P., Boivin, G., and Beauchemin, C. A. A. (2011a). Design considerations in building in silico equivalents of common experimental influenza virus assays and the benefits of such an approach. Autoimmunity, 44(4).

[39] Holder, B. P., Simon, P., Liao, L. E., Abed, Y., Bouhy, X., Beauchemin, C. A. A., and Boivin, G. (2011b). Assessing the in vitro fitness of an oseltamivir-resistant seasonal A/H1N1 influenza strain using a mathematical model. PLoS ONE, 6(3):e14767. 
[40] Hoopes, J. D., Driebe, E. M., Kelley, E., Engelthaler, D. M., Keim, P. S., Perelson, A. S., Rong, L., Went, G. T., and Nguyen, J. T. (2011). Triple combination antiviral drug (TCAD) composed of amantadine, oseltamivir, and ribavirin impedes the selection of drug-resistant influenza A virus. PLoS ONE, 6(12):e29778.

[41] Huang, A. S. (1973). Defective interfering viruses. Annu. Rev. Microbiol., 27:101118.

[42] Huang, A. S. and Baltimore, D. (1970). Defective viral particles and viral disease processes. Nature, 226(5243):325-327.

[43] Huang, I.-C., Li, W., Sui, J., Marasco, W., Choe, H., and Farzan, M. (2008). Influenza A virus neuraminidase limits viral superinfection. J. Virol., 82(10):48344843 .

[44] Hughes, M. T., Matrosovich, M., Rodgers, M. E., McGregor, M., and Kawaoka, Y. (2000). Influenza A viruses lacking sialidase activity can undergo multiple cycles of replication in cell culture, eggs, or mice. J. Virol., 74(11):5206-5212.

[45] Hussain, M., Galvin, H. D., Haw, T. Y., Nutsford, A. N., and Husain, M. (2017). Drug resistance in influenza A virus: the epidemiology and management. Infect. Drug Resist., 10:121-134.

[46] Iwami, S., Sato, K., De Boer, R. J., Aihara, K., Miura, T., and Koyanagi, Y. (2012). Identifying viral parameters from in vitro cell cultures. Front. Microbiol., 3:319.

[47] Jakubcová, L., Hollý, J., and Varečcková, E. (2016). The role of fusion activity of influenza a viruses in their biological properties. Acta Virol., 60(2):121-135.

[48] Janda, J. M., Davis, A. R., Nayak, D. P., and De, B. K. (1979). Diversity and generation of defective interfering influenza virus particles. Virology, 95(1):48-58.

[49] Jennings, P. A., Finch, J. T., Winter, G., and Robertson, J. S. (1983). Does the higher order structure of the influenza virus ribonucleoprotein guide sequence rearrangements in influenza viral RNA? Cell, 34(2):619-627.

[50] Johnston, R. E., Tovell, D. R., Brown, D. T., and Faulkner, P. (1975). Interfering passages of Sindbis virus: concomitant appearance of interference, morphological variants, and truncated viral RNA. J. Virol., 16(4):951-958. 
[51] Jorba, N., Coloma, R., and Ortín, J. (2009). Genetic trans-complementation establishes a new model for influenza virus RNA transcription and replication. PLoS Pathog., 5(5):e1000462.

[52] Kantorovich-Prokudina, E. N., Semyonova, N. P., Berezina, O. N., and Zhdanov, V. M. (1980). Gradual changes of influenza virions during passage of undiluted material. J. Gen. Virol., 50(1):23-31.

[53] Kawakami, E., Watanabe, T., Fujii, K., Goto, H., Watanabe, S., Noda, T., and Kawaoka, Y. (2011). Strand-specific real-time RT-PCR for distinguishing influenza vRNA, cRNA, and mRNA. J. Virol. Methods, 173(1):1-6.

[54] Khan, S. R. and Lazzarini, R. A. (1977). The relationship between autointerference and the replication of a defective interfering particle. Virology, 77(1):189-201.

[55] Kirkwood, T. B. L. and Bangham, C. R. M. (1994). Cycles, chaos, and evolution in virus cultures: A model of defective interfering particles. Proc. Natl. Acad. Sci. U.S.A., 91(18):8685-8689.

[56] Kiso, M., Mitamura, K., Sakai-Tagawa, Y., Shiraishi, K., Kawakami, C., Kimura, K., Hayden, F. G., Sugaya, N., and Kawaoka, Y. (2004). Resistant influenza A viruses in children treated with oseltamivir: descriptive study. Lancet, 364(9436):759-765.

[57] Kowal, K. J. and Strollar, V. (1980). Differential sensitivity of infectious and defective-interfering particles of sindbis virus to ultraviolet irradiation. Virology, 103(1):149-157.

[58] Kundu, A. and Nayak, D. P. (1994). Analysis of the signals for polarized transport of influenza virus (A/WSN/33) neuraminidase and human transferrin receptor, type II transmembrane proteins. J. Virol., 68(3):1812-1818.

[59] Lakadamyali, M., Rust, M. J., and Zhuang, X. (2004). Endocytosis of influenza viruses. Microbes Infect., 6(10):929-936.

[60] Laske, T., Heldt, F. S., Hoffmann, H., Frensing, T., and Reichl, U. (2016). Modeling the intracellular replication of influenza A virus in the presence of defective interfering RNAs. Virus Res., 213:90-99.

[61] Lee, B. Y., Haidari, L. A., and Lee, M. S. (2013). Modelling during an emergency: the 2009 H1N1 influenza pandemic. Clin. Microbiol. Infect., 19(11):1014-1022. 
[62] Li, T. C. M., Chan, M. C. W., and Lee, N. (2015). Clinical implications of antiviral resistance in influenza. Viruses, 7(9):4929-4944.

[63] Liu, C., Eichelberger, M. C., Compans, R. W., and Air, G. M. (1995). Influenza type A virus neuraminidase does not play a role in viral entry, replication, assembly, or budding. J. Virol., 69(2):1099-1106.

[64] Macdonald, R. D. and Yamamoto, T. (1978). Quantitative analysis of defective interfering particles in infectious pancreatic necrosis virus preparations. Arch. Virol., $57(1): 77-89$.

[65] Marcus, P. I., Ngunjiri, J. M., and Sekellick, M. J. (2009). Dynamics of biologically active subpopulations of influenza virus: Plaque-forming, noninfectious cell-killing, and defective interfering particles. J. Virol., 83(16):8122-8130.

[66] Marriott, A. C. and Dimmock, N. J. (2010). Defective interfering viruses and their potential as antiviral agents. Rev. Med. Virol., 20(1):51-62.

[67] Martin, K. and Heleniust, A. (1991). Nuclear transport of influenza virus ribonucleoproteins: The viral matrix protein (M1) promotes export and inhibits import. Cell, 67(1):117-130.

[68] McLain, L., Armstrong, S. J., and Dimmock, N. J. (1988). One defective interfering particle per cell prevents influenza virus-mediated cytopathology: an efficient assay system. J. Gen. Virol., 69(Pt 6):1415-1419.

[69] McLeod, B. and Burroughs, N. (2000). Defective deletion mutant amplification. J. Theor. Biol., 206(4):449-464.

[70] McVernon, J., McCaw, C., and Mathews, J. (2007). Model answers or trivial pursuits? the role of mathematical models in influenza pandemic preparedness planning. Influenza Other Respir. Viruses, 1(2):43-54.

[71] Mitchell, H., Levin, D., Forrest, S., Beauchemin, C. A. A., Tipper, J., Knight, J., Donart, N., Layton, R. C., Pyles, J., Gao, P., Harrod, K. S., Perelson, A. S., and Koster, F. (2011). Higher level of replication efficiency of 2009 (H1N1) pandemic influenza virus than those of seasonal and avian strains: Kinetics from epithelial cell culture and computational modeling. J. Virol., 85(2):1125-1135.

[72] Morens, D. M. and Taubenberger, J. K. (2011). Pandemic influenza: certain uncertainties. Rev. Med. Virol., 21(5):262-284. 
[73] Nayak, D. P. (1980). Defective interfering influenza viruses. Annu. Rev. Microbiol., 34:619-644.

[74] Nayak, D. P., Balogun, R. A., Yamada, H., Zhou, Z. H., and Barman, S. (2009). Influenza virus morphogenesis and budding. Virus Res, 143(2):147-161.

[75] Nayak, D. P., Tobita, K., Janda, J., Davis, A. R., and De, B. K. (1978). Homologous interference mediated by defective interfering influenza virus derived from a temperature-sensitive mutant of influenza virus. J. Virol., 28(1):375-386.

[76] Nelson, G. W. and Perelson, A. S. (1995). Modeling defective interfering virus therapy for AIDS: conditions for DIV survival. Math. Biosci., 125(2):127-153.

[77] Newcomb, L. L., Lin Kuo, R., Ye, Q., Jiang, Y., Tao, Y. J., and Krug, R. M. (2009). Interaction of the influenza A virus nucleocapsid protein with the viral RNA polymerase potentiates unprimed viral RNA replication. J. Virol., 83(1):29-36.

[78] Noda, T., Sagara, H., Yen, A., Takada, A., Kida, H., Cheng, R. H., and Kawaoka, Y. (2006). Architecture of ribonucleoprotein complexes in influenza A virus particles. Nature, 439(7075):490-492.

[79] Nunes-Correia, I., Ramalho-Santos, J., Nir, S., and Pedroso de Lima, M. C. (1999). Interactions of influenza virus with cultured cells: Detailed kinetic modeling of binding and endocytosis. Biochemistry, 38(3):1095-1101.

[80] Odagiri, T. and Tashiro, M. (1997). Segment-specific noncoding sequences of the influenza virus genome RNA are involved in the specific competition between defective interfering RNA and its progenitor RNA segment at the virion assembly step. J. Virol., $71(3): 2138-2145$.

[81] Paradis, E. G., Pinilla, L., Holder, B. P., Abed, Y., Boivin, G., and Beauchemin, C. A. A. (2015). Impact of the H275Y and I223V mutations in the neuraminidase of the 2009 pandemic influenza virus in vitro and evaluating experimental reproducibility. PLoS ONE, 10(5):e0126115.

[82] Perelson, A. S., Neumann, A. U., Markowitz, M., Leonard, J. M., and Ho, D. D. (1996). HIV-1 dynamics in vivo: Virion clearance rate, infected cell life-span, and viral generation time. Science, 271(5255):1582-1586.

[83] Perelson, A. S., Rong, L., and Hayden, F. G. (2012). Combination antiviral therapy for influenza: Predictions from modeling of human infections. J. Infect. Dis., 205(11):1642-1645. 
[84] Perez, J. T., Zlatev, I., Aggarwal, S., Subramanian, S., Sachidanandam, R., Kim, B., Manoharan, M., and tenOever, B. R. (2012). A small-RNA enhancer of viral polymerase activity. J. Virol., 86(24):13475-13485.

[85] Pinilla, L. T., Holder, B. P., Abed, Y., Boivin, G., and Beauchemin, C. A. A. (2012). The H275Y neuraminidase mutation of the pandemic A/H1N1 virus lengthens the eclipse phase and reduces viral output of infected cells, potentially compromising fitness in ferrets. J. Virol., 86(19):10651-10660.

[86] Popescu, M., Schaefer, H., and Lehmann-Grube, F. (1976). Homologous interference of lymphocytic choriomeningitis virus: Detection and measurement of interference focus-forming units. J. Virol., 20(1):1-8.

[87] Portela, A. and Digard, P. (2002). The influenza virus nucleoprotein: a multifunctional RNA-binding protein pivotal to virus replication. J. Gen. Virol., 83(Pt 4):723-734.

[88] Public Health Agency of Canada (Published May 2016). FluWatch report: May 14 to May 20, 2017 (week 20). Weekly influenza reports, Public Health Agency of Canada. Available online at: https://www.canada.ca/en/public-health/services/publications/ diseases-conditions/fluwatch/2016-2017/week20-may-14-20-2017.html.

[89] Ramratnam, B., Bonhoeffer, S., Binley, J., Hurley, A., Zhang, L., Mittler, J. E., Markowitz, M., Moore, J. P., Perelson, A. S., and Ho, D. D. (1999). Rapid production and clearance of HIV-1 and hepatitis $\mathrm{C}$ virus assessed by large volume plasma apheresis. Lancet, 354(9192):1782-1785.

[90] Resa-Infante, P., Jorba, N., Coloma, R., and Ortín, J. (2011). The influenza RNA synthesis machine: Advances in its structure and function. RNA Biol., 8(2):207-215.

[91] Rong, L., Dahari, H., Ribeiro, R. M., and Perelson, A. S. (2010). Rapid emergence of protease inhibitor resistance in hepatitis C virus. Sci. Transl. Med., 2(30):30ra32.

[92] Rouzine, I. M. and Weinberger, L. S. (2013). Design requirements for interfering particles to maintain coadaptive stability with HIV-1. J. Virol., 87(4):2081-2093.

[93] Roy, A.-M. M., Parker, J. S., Parrish, C. R., and Whittaker, G. R. (2000). Early stages of influenza virus entry into Mv-1 lung cells: Involvement of dynamin. Virology, $267(1): 17-28$. 
[94] Russell, C. A., Jones, T. C., Barr, I. G., Cox, N. J., Garten, R. J., Gregory, V., Gust, I. D., Hampson, A. W., Hay, A. J., Hurt, A. C., de Jong, J. C., Kelso, A., Klimov, A. I., Kageyama, T., Komadina, N., Lapedes, A. S., Lin, Y. P., Mosterin, A., Obuchi, M., Odagiri, T., Osterhaus, A. D. M. E., Rimmelzwaan, G. F., Shaw, M. W., Skepner, E., Stohr, K., Tashiro, M., Fouchier, R. A. M., and Smith, D. J. (2008). The global circulation of seasonal influenza a (H3N2) viruses. Science, 320(5874):340-346.

[95] Šantak, M., Markušić, M., Balija, M. L., Kopač, S. K., Jug, R., Övell, C., Tomac, J., and Forčić, D. (2015). Accumulation of defective interfering viral particles in only a few passages in Vero cells attenuates mumps virus neurovirulence. Microbes Infect., $17(3): 228-236$.

[96] Seitz, C., Frensing, T., Höper, D., Kochs, G., and Reichl, U. (2010). High yields of influenza A virus in Madin-Darby canine kidney cells are promoted by an insufficient interferon-induced antiviral state. J. Gen. Virol., 91(Pt 7):1754-1763.

[97] Shaw, M. L. and Palese, P. (2013). Orthomyxoviridae. In Knipe, D. M., Howley, P. M., Cohen, J. I., Griffin, D. E., Lamb, R. A., Martin, M. A., Racaniello, V. R., and Roizman, B., editors, Fields Virology, volume 1, chapter 40, pages 1151-1185. Lippincott Williams \& Wilkins, sixth edition.

[98] Simon, P. F., de La Vega, M.-A., Paradis, E., Mendoza, E., Coombs, K. M., Kobasa, D., and Beauchemin, C. A. A. (2016). Avian influenza viruses that cause highly virulent infections in humans exhibit distinct replicative properties in contrast to human H1N1 viruses. Sci. Rep., 6:24154.

[99] Smith, A. M. and Perelson, A. S. (2011). Influenza A virus infection kinetics: quantitative data and models. Wiley Interdiscip. Rev. Syst. Biol. Med., 3(4):429-445.

[100] Stegmann, T., Orsel, J. G., Jamieson, J. D., and Padfield, P. J. (1995). Limitations of the octadecylrhodamine dequenching assay for membrane fusion. Biochem. J., 307(Pt 3):875-878.

[101] Tarbet, E. B., Vollmer, A. H., Hurst, B. L., Barnard, D. L., Furuta, Y., and Smee, D. F. (2014). In vitro activity of favipiravir and neuraminidase inhibitor combinations against oseltamivir-sensitive and oseltamivir-resistant pandemic influenza A (H1N1) virus. Arch. Virol., 159(6):1279-1291.

[102] Thompson, K. A. S., Rempala, G. A., and Yin, J. (2009). Multiple-hit inhibition of infection by defective interfering particles. J. Gen. Virol., 90(Pt 4):888-899. 
[103] Treuhaft, M. W. and Beem, M. O. (1982). Defective interfering particles of respiratory syncytial virus. Infect. Immun., 37(2):439-444.

[104] Vreede, F. T. and Brownlee, G. G. (2007). Influenza virion-derived viral ribonucleoproteins synthesize both mRNA and cRNA in vitro. J. Virol., 81(5):2196-2204.

[105] Vreede, F. T., Jung, T. E., and Brownlee, G. G. (2004). Model suggesting that replication of influenza virus is regulated by stabilization of replicative intermediates. J. Virol., 78(17):9568-9572.

[106] Weinstock, D. M. and Zuccotti, G. (2006). Adamantane resistance in influenza A. JAMA, 295(8):934-936.

[107] Weinstock, D. M. and Zuccotti, G. (2009). The evolution of influenza resistance and treatment. JAMA, 301(10):1066-1069.

[108] Weiss, B. and Schlesinger, S. (1973). Defective interfering passages of Sindbis virus: Chemical composition, biological activity, and mode of interference. J. Virol., $12(4): 862-871$.

[109] Welsh, R. M., O’Connell, C. M., and Pfau, C. J. (1972). Properties of defective lymphocytic choriomeningitis virus. J. Gen. Virol., 17(3):355-359.

[110] Welsh, R. M. and Pfau, C. J. (1972). Determinants of defective lymphocytic choriomeningitis interference. J. Gen. Virol., 14(2):177-187.

[111] World Health Organization (Revised November 2016). Influenza. (Seasonal). Fact Sheet 211, World Health Organization. Available online at: http://www. who.int/mediacentre/factsheets/fs211/en/.

[112] Wright, P. F., Neumann, G., and Kawaoka, Y. (2013). Orthomyxoviruses. In Knipe, D. M., Howley, P. M., Cohen, J. I., Griffin, D. E., Lamb, R. A., Martin, M. A., Racaniello, V. R., and Roizman, B., editors, Fields Virology, volume 1, chapter 41, pages 1186-1243. Lippincott Williams \& Wilkins, sixth edition.

[113] Zheng, W. and Tao, Y. J. (2013). Structure and assembly of the influenza A virus ribonucleoprotein. FEBS Lett., 587(8):1206-1214.

[114] Ziegler, C. M., Eisenhauer, P., Bruce, E. A., Weir, M. E., King, B. R., and Klaus, J. P. (2016). The lymphocytic choriomeningitis virus matrix protein PPXY late domain drives the production of defective interfering particles. PLoS Pathog., 12(3):e1005501. 


\section{Glossary}

$\mathbf{A I C}_{\mathbf{C}}$ Akaike's information criterion (corrected).

B\&C Bellett \& Cooper.

cRNA complementary ribonucleic acid.

DIP Defective interfering particle.

DI RNA Defective interfering ribonucleic acid.

HA Hemagglutinin.

LIB Liao, Iwami, and Beauchemin.

MC Multiple-cycle.

MDCK Madin Darby canine kidney.

MM Mathematical model.

MOI Multiplicity of infection.

MY Mock-yield.

mRNA Messenger ribonucleic acid.

NA Neuraminidase.

NAI Neuraminidase inhibitor.

NEP Nuclear export protein.

NP Nucleoprotein.

ODE Ordinary differential equation. 
PA Polymerase acid.

PB1 Polymerase basic 1.

PB2 Polymerase basic 2 .

PFU Plaque-forming unit.

RNP Ribonucleoprotein.

SC Single-cycle.

SSR Sum-of-squared residuals.

STV Standard virus.

vRNA viral ribonucleic acid. 